\title{
Silicate features in Galactic and extragalactic post-AGB discs ${ }^{\star} \star \star$
}

\author{
C. Gielen ${ }^{1,2, \star \star \star}$, J. Bouwman ${ }^{2}$, H. Van Winckel ${ }^{1}$, T. Lloyd Evans ${ }^{3}$, P. M. Woods ${ }^{4,5}$, \\ F. Kemper ${ }^{6,4}$, M. Marengo ${ }^{7}$, M. Meixner ${ }^{8}$, G. C. Sloan ${ }^{9}$, and A. G. G. M. Tielens ${ }^{10}$
}

1 Instituut voor Sterrenkunde, Katholieke Universiteit Leuven, Celestijnenlaan 200D, 3001 Leuven, Belgium

e-mail: clio.gielen@ster. kuleuven. be

2 Max Planck Institut für Astronomie, Königstuhl 17, 69117 Heidelberg, Germany

3 SUPA, School of Physics and Astronomy, University of St Andrews, North Haugh, St Andrews, Fife KY16 9SS, UK

4 Jodrell Bank Centre for Astrophysics, Alan Turing Building, School of Physics and Astronomy, The University of Manchester, Oxford Road, Manchester M13 9PL, UK

5 Department of Physics and Astronomy, University College London, Gower Street, London, WC1E 6BT, UK

${ }^{6}$ Institute of Astronomy and Astrophysics, Academia Sinica, PO Box 23-141, Taipei 10617, Taiwan, R. O. C.

7 Department of Physics and Astronomy, Iowa State University, A313E Zaffarano, Ames, IA 50010, USA

8 Space Telescope Science Institute, 3700 San Martin Drive, Baltimore, MD 21218, USA

9 Department of Astronomy, Cornell University, Ithaca, NY 14853, USA

10 Leiden Observatory, Leiden University, PO Box 9513, 2300 RA Leiden, The Netherlands

Received 29 May 2011 / Accepted 2 August 2011

\section{ABSTRACT}

\begin{abstract}
Aims. In this paper we study the Spitzer and TIMMI2 infrared spectra of post-AGB disc sources, both in the Galaxy and the LMC. Using the observed infrared spectra we determine the mineralogy and dust parameters of the discs, and look for possible differences between the Galactic and extragalactic sources.

Methods. Modelling the full spectral range observed allows us to determine the dust species present in the disc and different physical parameters such as grain sizes, dust abundance ratios, and the dust and continuum temperatures.

Results. We find that all the discs are dominated by emission features of crystalline and amorphous silicate dust. Only a few sample sources show features due to $\mathrm{CO}_{2}$ gas or carbonaceous molecules such as PAHs and $\mathrm{C}_{60}$ fullerenes. Our analysis shows that dust grain processing in these discs is strong, resulting in large average grain sizes and a very high crystallinity fraction. However, we do not find any correlations between the derived dust parameters and properties of the central source. There also does not seem to be a noticeable difference between the mineralogy of the Galactic and LMC sources. Even though the observed spectra are very similar to those of protoplanetary discs around young stars, showing similar mineralogy and strong grain processing, we do find evidence for differences in the physical and chemical processes of the dust processing.
\end{abstract}

Key words. stars: AGB and post-AGB - stars: evolution - circumstellar matter - binaries: general

\section{Introduction}

Studies of the chemistry and geometry of circumstellar discs have, so far, mainly focussed on the protoplanetary discs around young stars (e.g. Meeus et al. 2001; Bouwman et al. 2008; Juhász et al. 2010). However, in recent years it became clear that circumstellar discs are present in nearly all stages of stellar evolution, going from first-ascent giants (Jura 2003; Verhoelst et al. 2007; Melis et al. 2010), B[e] supergiants (Kastner et al. 2010), asymptotic giant branch (AGB) stars (e.g. Yamamura et al. 2000; Chiu et al. 2006; Deroo et al. 2007), (proto-)planetary nebulae (e.g. Chesneau et al. 2006, 2007; Lykou et al. 2011) to white dwarves (e.g. Becklin et al. 2005; Dong et al. 2010). Even though circumstellar discs appear common throughout the Hertzsprung-Russell diagram, it is still unclear what links the

* Based on observations obtained at the European Southern Observatory (ESO), La Silla, observing program 072.D-0263 and 077.D-0555, and on observations made with the Spitzer Space Telescope (program id 3274 and 50092), which is operated by the Jet Propulsion Laboratory, California Institute of Technology under a contract with NASA

$\star \star$ Appendix $\mathrm{A}$ is available in electronic form at

http://www . aanda. org

$\star \star \star$ Postdoctoral Fellow of the Fund for Scientific Research, Flanders. different disc-bearing objects throughout all the late evolutionary stages. It is likely that there are different formation channels depending on the evolutionary status of the central object.

Whereas for young stars the disc is a by-product of the star formation, there is evidence that for the majority of the evolved stars the disc is newly formed. The exact formation mechanisms are unknown, and will most likely differ for different evolutionary stages. For example, disc formation has been linked to binary mergers, wind capture or Roche-lobe overflow (see references above). However, in most cases, binarity appears to be the key ingredient to the formation of discs in later stages of stellar evolution.

In this work we study a particular class of evolved binary post-AGB stars surrounded by stable dusty discs. These sources were initially selected on the basis of their very strong nearinfrared excess. Follow-up studies confirmed the binarity, and showed that the companion star is most likely a main-sequence star, with a typical separation of about 1 AU (Van Winckel et al. 2009). The presence of a disc was already proposed to explain the presence of hot dust in the system (De Ruyter et al. 2006) and later resolved by interferometric observations (Bujarrabal et al. 2001, 2007; Deroo et al. 2006; Deroo 2007). The discs also explain the observed depletion process in the photospheric 
abundances of the central post-AGB star (Waters et al. 1992; Maas et al. 2005; Gielen et al. 2009b), Since the dust sublimation radii for these sources are well beyond the orbit, all the discs are circumbinary.

Our previous studies have shown that the discs are ideal environments for strong dust processing, in the form of grain growth and crystallisation (Gielen et al. 2008, 2009a,b). This dust composition is very similar to what is observed for protoplanetary discs around young stars, even though the disc formation mechanisms, and probably also the initial dust species, are very different.

In the Galaxy, around 80 such systems are now known (De Ruyter et al. 2006). Recently, large programmes, such as the Spitzer SAGE (Surveying the Agents of Galaxy Evolution) photometric (Meixner et al. 2006), and follow-up SAGE-Spec spectroscopic (Kemper et al. 2010), programmes indicate that also in the Large Magellanic Cloud (LMC) post-AGB disc sources are common: The study of van Aarle et al. (2011) lists about 650 probable post-AGB disc candidates in the LMC, and about the same number for post-AGB stars surrounded by a cool expanding dust shell, using SAGE photometric data.

In this paper we look in more detail to the mineralogy of the circumbinary discs, both for sources in the Galaxy and in the LMC. For this we use high- and low-resolution Spitzer and TIMMI 2 infrared spectra. These spectra allow us to study dust and gas emission features in the $5-35 \mu \mathrm{m}$ region.

The outline of the paper is as follows: in Sects. 2 and 3 we describe the selected samples Galactic and LMC stars and the data reduction process. In Sect. 4 we take a first look at the different emission features in individual sources, and compare the Galactic and LMC sample. The results on the dust parameters using a more detailed model to fit the full Spitzer wavelength range are described in Sect. 5. Finally, we end with a discussion and conclusions in Sects. 6 and 7.

\section{Programme stars}

In this paper we study a total of 57 post-AGB stars with evidence for the presence of a stable circumbinary disc, located in the Galaxy and the LMC. The Galactic sample consists of 33 stars from the larger sample discussed in De Ruyter et al. (2006). Of these stars, 21 sources are already discussed in Gielen et al. (2008) and Gielen et al. (2009a). To complement these 21 sources we obtained Spitzer high- and low-resolution spectra of 13 additional suspected post-AGB disc sources. The LMC sample consists of 24 sources, of which 3 are already discussed briefly in Gielen et al. (2009b). These sources were observed in low-resolution mode, either as part of the larger SAGE-Spec programme of Kemper et al. (2010), a follow-up to the photometric SAGE legacy programme (Meixner et al. 2006), or as part of Spitzer programmes 3274 (PI: Hans Van Winckel) and 50092 (PI: Clio Gielen) ${ }^{1}$.

From Woods et al. (2011), we selected the stars which are classified as oxygen-rich post-AGB or RV Tauri sources in the SAGE-Spec catalogue. We removed the sources for which only a small part of the Spitzer wavelength range was observed (LH $\alpha$ 120-N 145 and MACHO 81.9728.14). After this, 16 stars remained. To increase this LMC sample, we searched the SAGE photometric catalogue for the presence of other possible disc bearing post-AGB sources. All objects with $24 \mu \mathrm{m}$ fluxes between $2 \mathrm{mJy}$ and $1 \mathrm{Jy}$ were selected, in order to exclude young

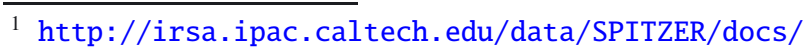

stellar objects and supergiants. Other selection criteria were chosen to distinguish between post-AGB stars with an expanding shell $\left(F_{24}>\mathrm{F}_{8}\right)$ and binary post-AGB sources with a circumbinary disc $\left(F_{24}>0.5 F_{8}\right.$ and $\left.J-K<1\right)$. For a detailed description of the selection criteria we refer to van Aarle et al. (2011). After cross-correlation with optical photometric catalogues and the SIMBAD Astronomical Database, 650 sources remained. Of this larger sample, the 8 brightest stars were selected and observed with the Spitzer infrared spectrograph. For 18 of the 24 LMC sources additional ground-based optical spectra were obtained at Siding Spring Observatory, the South African Astronomical Observatory (SAAO) or with the UVES spectrograph in Paranal. This allows us to determine a spectral type and assign an effective temperature (Gielen et al. 2009b; van Aarle et al. 2011).

For 15 Galactic sources the binarity has been confirmed by radial velocity monitoring, resulting in orbital periods between 200 and 1800 days (Van Winckel et al. 2009). For the other Galactic sources, binarity can already be suspected from the monitoring programme but not enough data are available to derive the exact orbital parameters. Unfortunately, such longterm radial velocity monitoring programme for the LMC sources is difficult, since it requires several years of observations with a high-resolution optical spectrograph on a large telescope, such as UVES on the VLT. But, given the strong resemblance of the LMC disc candidates to the Galactic disc sources, in chemistry of the central star, spectral energy distribution (SED) and mineralogy of the circumstellar environment (Reyniers \& Van Winckel 2007; Gielen et al. 2009b), we postulate that these sources will also be part of a binary system.

\subsection{Spectral energy distribution}

For all Galactic sample stars, SEDs were calculated from the photometric data and stellar parameters as given in De Ruyter et al. (2006), the SAGE photometric catalogue and/or the Vizier database. The resulting SEDs can be seen in Fig. 1. From the SED we also calculated the luminosity ratio $L_{\mathrm{IR}} / L_{*}$. The total extinction $E(B-V)_{\text {tot }}$ was determined by dereddening the observed photometry and infrared spectra, using the average extinction law of Savage \& Mathis (1979) extended with the theoretical extinction law of Steenman \& Thé (1989, 1991). Minimising the difference between the dereddened observed optical fluxes and the appropriate Kurucz model (Kurucz 1979) gives the total colour excess $E(B-V)_{\text {tot }}$ (Tables 1-2). This is done under the assumption that the extinction is fully due to interstellar extinction, or that the circumstellar component follows the same extinction law. Since the total extinction probably consists of both an interstellar and a circumstellar component, the applied dereddening is thus a maximal correction. The errors on the value for $E(B-V)_{\text {tot }}$ are calculated using a Monte-Carlo simulation on the photometric data. We use an error of 0.05 for the photometric measurements in a Gaussian distribution. Since we do not know the distances to the Galactic sources, we adopt a likely luminosity for evolved low-gravity objects of $L_{*}=5000 \pm 2000 L_{\odot}$. For the LMC sources we calculate the luminosity assuming a typical LMC distance of 50000 pc (Kemper et al. 2010). For the LMC sources we use the effective temperatures as given in van Aarle et al. (2011), if available. Since the metallicity and $\log g$ values for these sources are not determined, we used values of $[\mathrm{Fe} / \mathrm{H}]=-1.0$ and $\log g=1.0$ for all stars. These values are consistent with those found for the Galactic objects, and have only minimal impact on the derived total reddening and infrared energy ratio. Of the LMC sample, 6 sources lack optical spectra, 
C. Gielen et al.: Silicate features in Galactic and extragalactic post-AGB disks

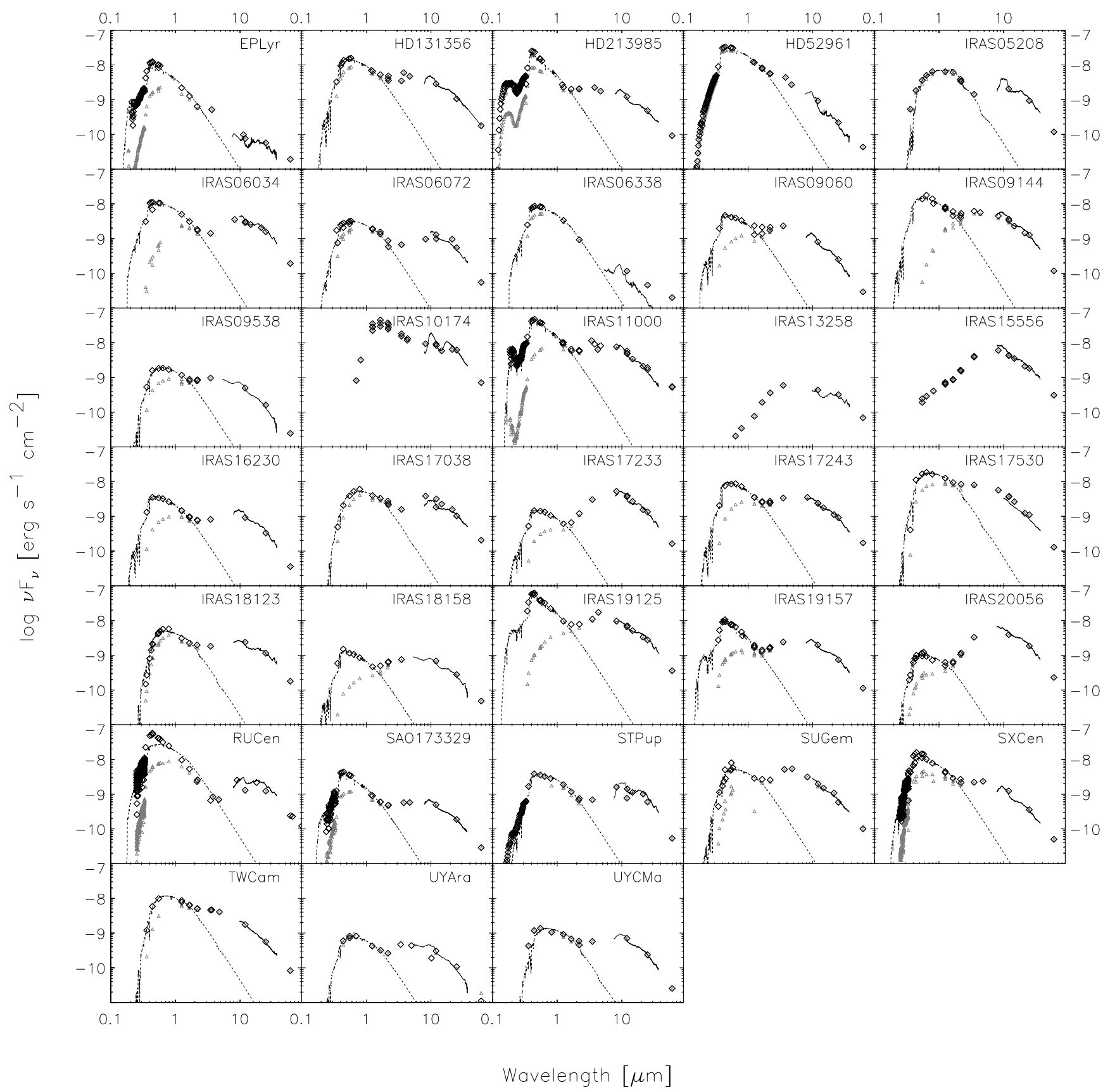

Fig. 1. The spectral energy distributions of our sample stars. The dereddened fluxes (diamonds), reddened fluxes (gray triangles) and Spitzer spectra (solid line) are given together with the scaled photospheric Kurucz model (dashed line). For the sources where we lack the stellar parameters to determine the underlying Kurucz model, we only plot the reddened data.

and thus effective temperatures, and we could not determine the total reddening.

For some sources there is evidence that the discs are seen close to edge on (Menzies \& Whitelock 1988; Lloyd Evans 1997). The visible light of the central source is then seen in reflection which makes an accurate determination of the total extinction, the luminosity ratio, and the distance very difficult. These sources are marked with an asterisk in Table 1.

\section{Observations and data reduction}

\subsection{Spitzer}

The spectra were obtained using the SL $(\lambda=5.3-14.5 \mu \mathrm{m})$, $\mathrm{LL}(\lambda=14-38 \mu \mathrm{m})$, $\mathrm{SH}(\lambda=9.9-19.5 \mu \mathrm{m})$ and $\mathrm{LH}$
( $\lambda=19.3-37 \mu \mathrm{m})$ staring modes on the Spitzer-IRS instrument (Werner et al. 2004; Houck et al. 2004). For the Galactic objects, exposure times were chosen to achieve a $S / N$ ratio of 400 . For the extragalactic objects in our own observing proposal, exposure times were chosen to give a $S / N$ ratio of 100 for the SL mode and 20 for the LL mode. The SAGE-Spec LMC objects have a $S / N$ ratio $\sim 60$ in SL mode and $\sim 30$ in LL mode.

The newly obtained spectra from our own Spitzer observations were extracted from the SSC raw data pipeline S18.0 version products, using the $\mathrm{c} 2 \mathrm{~d}$ and feps data reduction packages. For a detailed description of these reduction packages, we refer to Lahuis et al. (2006) and Hines et al. (2005). The reduction includes background and bad-pixel correction, extraction, defringing and order matching. Individual orders are corrected for offsets, if necessary, by applying small scaling corrections to match 


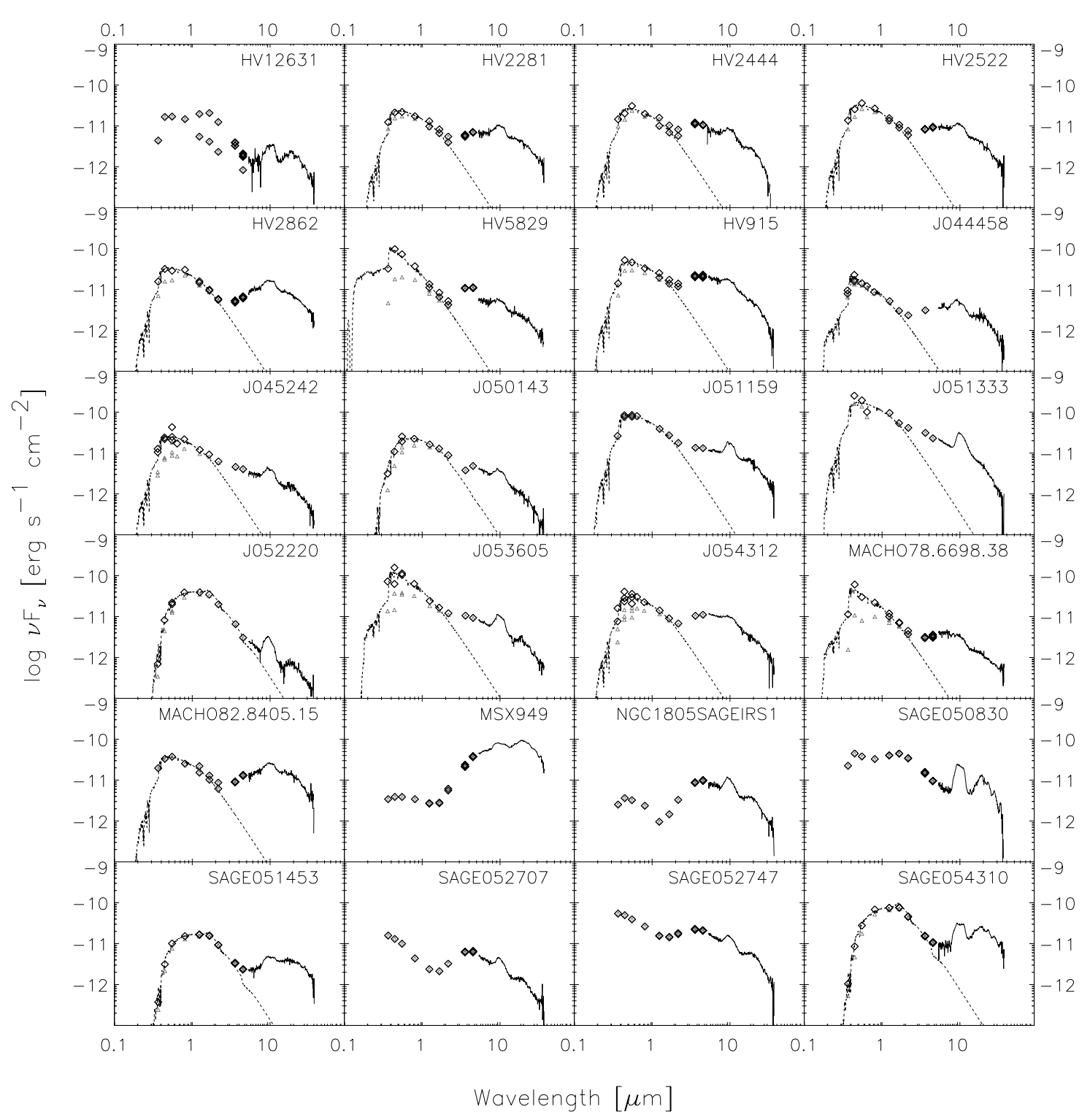

Fig. 1. continued.

the bluer order. For a detailed description of the target selection, observing strategy and reduction of the SAGE-Spec objects, we refer to Kemper et al. (2010).

\section{2. $T I M M I 2$}

For some stars we lack the Spitzer IRS-SH observations and we obtained additional ground-based $N$-band infrared spectra with the Thermal Infrared Multi Mode Instrument 2 (TIMMI2, Reimann et al. 2000; Käufl et al. 2003), mounted on the $3.6 \mathrm{~m}$ telescope at the ESO La Silla Observatory. The low-resolution $(R \sim 160) N$ band grism was used in combination with a 1.2 arcsec slit; the pixel scale in the spectroscopic mode of TIMMI 2 is 0.45 arcsec. For the reduction of the spectra we used the method described in van Boekel et al. (2005). We scaled the TIMMI2 spectra to the Spitzer spectra and found a very good agreement in spectral shape between the two data sets.

The resulting spectra can be found in Fig. 2 and Figs. A.6, A.7.

\section{First inspection of emission features}

Looking at the spectra of the Galactic and LMC sources (Figs. A.6, A.7), we find that all sources show clear silicate emission. For nearly all sources the prominent broad amorphous silicate features at 10 and $20 \mu \mathrm{m}$ stand out. Furthermore, most spectra show additional narrower features at 11.3,6,19,23,27 and $33 \mu \mathrm{m}$, which are due to crystalline silicate emission.

Even though all the spectra are dominated by oxygen-rich dust species, some stars do show evidence for the presence of carbonaceous molecules. Clear PAH emission can be seen in EP Lyr, IRAS 06338 and IRAS 13258, with peaks at 8 and $11.2 \mu \mathrm{m}$. The PAH features of EP Lyr were already discussed in Gielen et al. (2009a). The peculiar spectrum of IRAS 06338 not only shows the typical PAH bands, but several smaller features between 6 and $8 \mu \mathrm{m}$, most likely resulting from very small PAH grains. In this star, the strong narrow peaks between 13 and $18 \mu \mathrm{m}$ are due to $\mathrm{CO}_{2}$ gas emission, which can also be seen in EP Lyr and HD 52961. HD 52961 and IRAS 06338 both 
C. Gielen et al.: Silicate features in Galactic and extragalactic post-AGB disks

Table 1. Name, equatorial coordinates $\alpha$ and $\delta$ (J2000), effective temperature $T_{\text {eff }}$, surface gravity $\log g$ and metallicity [Fe/H] of our Galactic sample stars.

\begin{tabular}{|c|c|c|c|c|c|c|c|c|c|c|c|}
\hline $\mathrm{N}^{\circ}$ & Name & $\begin{array}{c}\alpha(\mathrm{J} 2000) \\
(\mathrm{h} \mathrm{m} \mathrm{s})\end{array}$ & $\begin{array}{c}\delta(\mathrm{J} 2000) \\
\left({ }^{\circ},{ }^{\prime \prime \prime}\right)\end{array}$ & $\begin{array}{l}T_{\text {eff }} \\
(\mathrm{K})\end{array}$ & $\begin{array}{l}\log g \\
(\operatorname{cgs})\end{array}$ & $\mathrm{e} / \mathrm{H}]$ & $\begin{array}{c}P_{\text {orbit }} \\
\text { (days) }\end{array}$ & $E(B-V)_{\mathrm{tot}}$ & $\begin{array}{c}L_{\mathrm{IR}} / L_{*} \\
(\%)\end{array}$ & $\begin{array}{c}d \\
(\mathrm{kpc})\end{array}$ & Prog. ID \\
\hline 1 & EP Lyr & 191817.5 & +275038 & 7000 & 2.0 & -1.5 & & $0.52 \pm 0.01$ & $3 \pm 0$ & $4.1 \pm 0.8$ & 3274 \\
\hline 2 & HD 131356 & & 23 & 6000 & 1.0 & -0.5 & 1490 & $0.20 \pm 0.01$ & & $.0 \pm 0.6$ & 3274 \\
\hline 3 & & & & & & .0 & & & & & 3274 \\
\hline 4 & 961 & & +10 & 00 & & -4.8 & 1310 & & & & 3274 \\
\hline 5 & $5208-2035$ & & & 00 & 5 & 0 & & & & & 3274 \\
\hline 6 & $6034+13$ & 3 & +135 & 6000 & 1.5 & -2.0 & & & & 0.7 & 50092 \\
\hline 7 & $6072+$ & 4 & +095 & & 0 & -2.0 & & & & & 0092 \\
\hline 8 & $6338+$ & $06 ?$ & 02 & 625 & 1.0 & -1.5 & & 02 & $3 \pm 0$ & 0.8 & 50092 \\
\hline 9 & $9060-$ & 09( & -28 & 6 & 1.5 & -0.5 & 371 & 02 & & 1.1 & 3274 \\
\hline 10 & & & & & 5 & -0.5 & 1770 & & & & 3274 \\
\hline 11 & & & & 5500 & 1.0 & -0.5 & & 0.35 & $64 \pm 5$ & $.8 \pm$ & 50092 \\
\hline 12 & IRAS 10174- & 101 & -571 & G8IaO & & & 323 & & & & 3274 \\
\hline 13 & IRAS $11000-615$ & 110 & -620943 & 7600 & 2.0 & 0.1 & & $0.63 \pm 0.01$ & $42 \pm 2$ & $1.9 \pm 0.4$ & 50092 \\
\hline 14 & IRAS $13258-81$ & & & F4Ib-G0Ib & & & & & & & 50092 \\
\hline 15 & & & & F8 & & & & & & & 50092 \\
\hline 16 & & & & & & -0.5 & & & & & 3274 \\
\hline 17 & & & & & & .5 & 1381 & & & & 274 \\
\hline 18 & & & & & & -1.0 & & 02 & 548 & .0 & 50092 \\
\hline 19 & & & & & & 0.0 & 484 & & & .8 & 327 \\
\hline 20 & & & & & 0.0 & 0.0 & & 02 & & .5 & 50092 \\
\hline 21 & & & & & 0.5 & 0.0 & & 02 & & .0 & 50092 \\
\hline 22 & & & & & 1. & 0.0 & & & & 3 & 50092 \\
\hline 23 & & & & & 1. & -0.5 & 517 & & & .4 & 3274 \\
\hline 24 & & & & & & 0.0 & 120.5 & & & & 3274 \\
\hline 25 & IF & & & & 07 & -0.4 & & 0.51 & $905 \pm 42$ & 10.9 & 3274 \\
\hline 26 & & & & & 1 & -2.0 & 148 & 0.55 & & & 3274 \\
\hline 27 & 017332 & & & & 1. & -0.8 & 115.9 & & & $6.5 \pm$ & 3274 \\
\hline 28 & 1 up & & & & 0.5 & -1.5 & 410 & $0.00 \pm($ & $55 \pm 1$ & $5.7 \pm 1.2$ & 3274 \\
\hline 29 & ) & & & & 1.125 & -0.7 & & & $111 \pm 7$ & $4.8 \pm 1.0$ & 3274 \\
\hline 30 & (2) & 122 & -491241 & & 1.0 & -1.0 & 600 & $0.32 \pm 0.02$ & & $3.8 \pm 0.7$ & 3274 \\
\hline 31 & TW Cam & & & & & -0.5 & & & & $3.2 \pm 0.6$ & 3274 \\
\hline 32 & & & & & & -1.0 & & & & $12 \pm 2.5$ & 50092 \\
\hline 33 & UY CMa* & 061 & -170235 & 5500 & 1.0 & 0.0 & & $0.00 \pm 0.00$ & $89 \pm 3$ & $9.6 \pm 2.0$ & 3274 \\
\hline
\end{tabular}

Notes. For the model parameters we refer to De Ruyter et al. (2006). Also given is the orbital period (see references in De Ruyter et al. 2006; Gielen et al. 2007; Van Winckel et al. 2009). The total reddening $E(B-V)_{\mathrm{tot}}$, the energy ratio $L_{\mathrm{IR}} / L_{*}$ and the calculated distance, assuming a luminosity of $L_{*}=5000 \pm 2000 L_{\odot}$. Stars marked with * are seen in reflection only, resulting in unreliable $E(B-V)_{\text {tot }}$ values and luminosity ratios, and upper limits for the distances. The last column lists whether the spectra are part of the SAGE-Spec catalogue, Spitzer programme 3274 or 50092 .
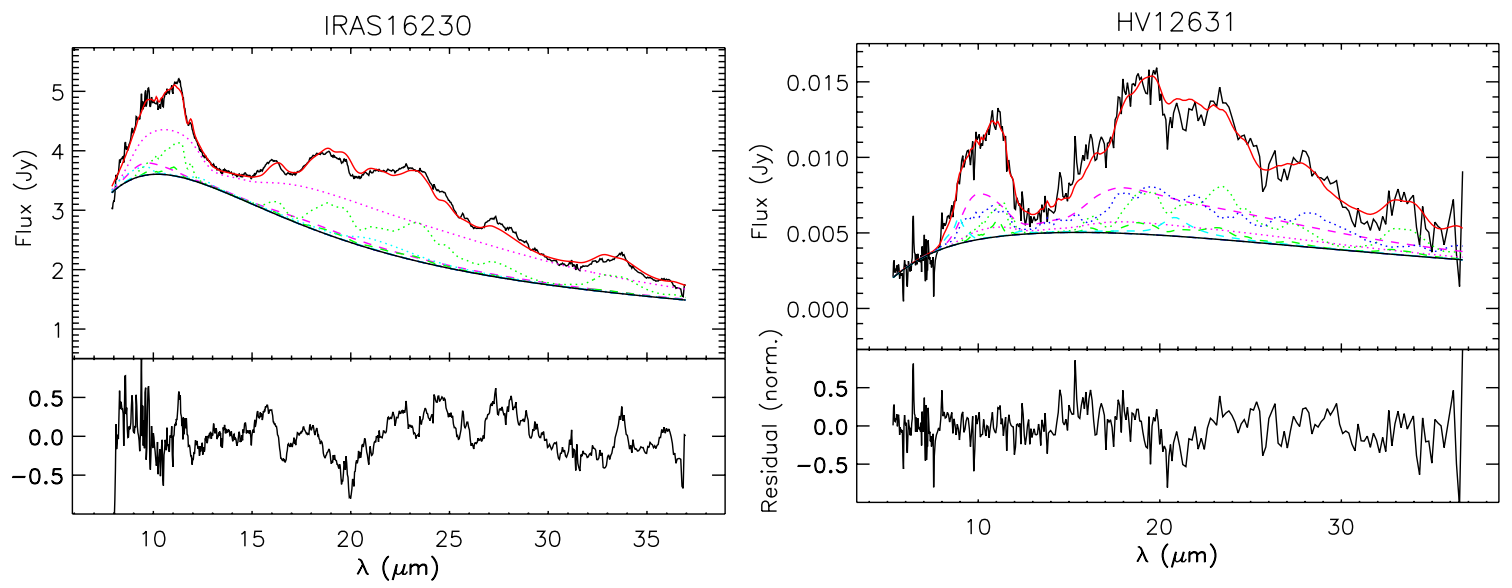

Fig. 2. Best model fits for two of our sample stars, showing the contribution of the different dust species. Top: the observed spectrum (black curve) is plotted together with the best model fit (red curve) and the continuum (black solid line). Forsterite is plotted in green, enstatite in blue, silica in cyan and amorphous olivine and pyroxene in magenta. Small grains $(0.1 \mu \mathrm{m})$ are plotted as dashed lines and larger grains $(2$ and $4 \mu \mathrm{m})$ as dotted lines. Bottom: the normalised residuals after subtraction of our best model of the observed spectra. The models for the other sample stars can be found in Figs. A.6, A.7. 
Table 2. Name, equatorial coordinates $\alpha$ and $\delta$ (J2000), and effective temperature $T_{\text {eff }}$ our LMC sample stars, taken from (van Aarle et al. 2011).

\begin{tabular}{|c|c|c|c|c|c|c|c|c|}
\hline $\mathrm{N}^{\circ}$ & Name & $\begin{array}{c}\alpha(\mathrm{J} 2000) \\
(\mathrm{h} \mathrm{m} \mathrm{s})\end{array}$ & $\begin{array}{c}\delta(\mathrm{J} 2000) \\
\left({ }^{\circ},{ }^{\prime \prime}\right)\end{array}$ & $\begin{array}{l}T_{\text {eff }} \\
(\mathrm{K})\end{array}$ & $\begin{array}{l}L_{*} \\
L_{\odot}\end{array}$ & $E(B-V)_{\text {tot }}$ & $\begin{array}{c}L_{\mathrm{IR}} / L_{*} \\
(\%)\end{array}$ & Prog. ID \\
\hline 34 & HV 12631 & 053933.1 & -712155 & & & & & SAGE-Spec \\
\hline 35 & HV 2281 & 050305.0 & -684025 & 5750 & 2000 & $0.02 \pm 0.02$ & $63 \pm 2$ & SAGE-Spec \\
\hline 36 & HV 2444 & 051846.0 & -690322 & 6750 & 4000 & $0.26 \pm 0.02$ & & SAGE-Spec \\
\hline 37 & HV 2522 & 052627.2 & -664259 & 6250 & 3700 & $0.17 \pm 0.02$ & $45 \pm 3$ & SAGE-Spec \\
\hline 38 & HV 2862 & 055121.1 & -695347 & 5750 & 2700 & $0.09 \pm 0.02$ & $54 \pm 2$ & SAGE-Spec \\
\hline 39 & HV 5829 & 052519.3 & -705407 & 5500 & 1800 & $0.00 \pm 0.02$ & $60 \pm 2$ & SAGE-Spec \\
\hline 40 & HV 915 & 051418.0 & -691235 & 6250 & 4600 & $0.21 \pm 0.01$ & $64 \pm 3$ & SAGE-Spec \\
\hline 41 & J044458.18-703522.8 & 044458.4 & -703523 & 7000 & 1400 & $0.04 \pm 0.02$ & $40 \pm 2$ & 50092 \\
\hline 42 & J045242.93-704737.4 & 045243.2 & -704737 & 5500 & 2500 & $0.30 \pm$ & $22 \pm 3$ & 50092 \\
\hline 43 & J050143.18-6940 & 43.5 & -694048 & 5000 & 2700 & & \pm 2 & 50092 \\
\hline 44 & J051159.11-692532.8 & 051159.4 & -692533 & 6250 & 8500 & 0.02 & $23 \pm 2$ & 50092 \\
\hline 45 & J051333.74-663419.1 & 051333.7 & -664319 & 6500 & 17000 & 0.12 & $18 \pm 2$ & 50092 \\
\hline 46 & J052220.87-655551.6 & 052221.1 & -655552 & 4250 & 5000 & 0.16 & $2 \pm 2$ & 50092 \\
\hline 47 & J053605.56-695802.9 & 053605.9 & -695803 & 6750 & 8500 & $0.38 \pm 0.03$ & $15 \pm 3$ & 50092 \\
\hline 48 & J054312.52-683356.9 & 054312.9 & -683357 & 6250 & 3000 & $0.27 \pm 0.02$ & $49 \pm 5$ & 50092 \\
\hline 49 & МАСНО 78.6698.38 & 052149.1 & -700434 & 7000 & 3300 & $0.46 \pm 0.03$ & $27 \pm 2$ & SAGE-Spec \\
\hline 50 & MACHO 82.8405.15 & 053150.9 & -691146 & 6000 & 3600 & $0.05 \pm 0.01$ & $84 \pm 3$ & SAGE-Spec \\
\hline 51 & MSX 949 & 054014.8 & -692849 & & & & & SAGE-Spec \\
\hline 52 & NGC 1805 SAGE IRS 1 & 050224.2 & -660637 & & & & & SAGE-Spec \\
\hline 53 & SAGE050830 & 050830.6 & -692237 & & & & & SAGE-Spec \\
\hline 54 & SAGE051453 & 051418.2 & -691724 & 4250 & 2100 & $0.10 \pm 0.02$ & $28 \pm 2$ & SAGE-Spec \\
\hline 55 & SAGE052707 & 052707.2 & -702002 & & & & & SAGE-Spec \\
\hline 56 & SAGE052747 & 052747.6 & -714853 & & & & & SAGE-Spec \\
\hline 57 & SAGE054310 & 054310.9 & -672728 & 4000 & 10500 & $0.32 \pm 0.02$ & $27 \pm 2$ & SAGE-Spec \\
\hline
\end{tabular}

Notes. The total reddening $E(B-V)_{\text {tot }}$, the energy ratio $L_{\mathrm{IR}} / L_{*}$, and the luminosity as calculated from our SED modelling. The last column lists whether the spectra are part of the SAGE-Spec catalogue, Spitzer programme 3274 or 50092.

show a strong feature at $18.7 \mu \mathrm{m}$, which can be identified as $\mathrm{C}_{60}$ fullerene emission (Cami et al. 2010). The detection of these carbonaceous molecules in our sample stars will be further discussed in an upcoming paper.

To study the silicate signatures in the infrared spectra, we divided the full spectrum into 7 different complexes where strong silicate emission is seen, more specifically at $10-14-16-$ $19-23-27$ and $33 \mu \mathrm{m}$. To compare the Galactic stars to the LMC sources, we calculated for each group a mean continuumsubtracted spectrum in these 7 complexes. The continuum was determined by linearly interpolating between the beginning and end of the studied regions. These mean spectra are then normalised to the maximum flux in the wavelength interval. An overview of the different mean spectra can be seen in Fig. 3, together with synthetic spectra of crystalline and amorphous silicates. Below we discuss the different complexes in more detail, and results can be seen in Fig. 5 and Figs. A.1 to A.5.

\subsection{The $10 \mu \mathrm{m}$ complex $(8-13 \mu \mathrm{m})$}

It is clear from Fig. 5 that the mean spectrum in this region is very similar for the Galactic and LMC sources. A flat-topped feature is seen, where the two peaks come from the emission of amorphous and crystalline olivine. The observed $10 \mu \mathrm{m}$ complex seems to be broader at the left shoulder, compared to the emission feature of amorphous olivine. Additional emission near $9 \mu \mathrm{m}$ could point to the presence of amorphous pyroxene or silica, which peak at shorter wavelengths.

Some individual sources do not follow the calculated mean complex. IRAS 13258 and EP Lyr show no silicate features, but exhibit emission due to PAHs. PAH emission probably also contributes to features seen in IRAS 06338 and HD 52961. Also note that in IRAS 06338, the strong feature at $9 \mu \mathrm{m}$ seems to be shifted bluewards in comparison to the mean. This could point to the dominance of silica in this source. IRAS 10174 shows almost no emission of crystalline species, not only at $10 \mu \mathrm{m}$ but along its entire wavelength range, and is very similar to the extragalactic source J 051333 . These two sources also do not show the broadening at the left shoulder of the complex, and are thus expected to be devoid of silica.

The $10 \mu \mathrm{m}$ complex is a good tracer of grain processing, in the form of grain size and crystallisation (van Boekel et al. 2003, 2005; Juhász et al. 2010). Since amorphous and crystalline silicates peak at two distinct wavelengths, respectively 9.8 and $11.3 \mu \mathrm{m}$, the continuum-subtracted $11.3 / 9.8 \mu \mathrm{m}$ flux can be used as a measure for the amount for the crystallisation the dust has undergone. Furthermore, the peak-to-continuum ratio of the $10 \mu \mathrm{m}$ complex can be used as a tracer for grain growth, since larger grains will result in a less pronounced feature. In Fig. 4 we plot these two ratios. We do not plot EP Lyr and IRAS 13258, since they show strong PAH emission at $11.3 \mu \mathrm{m}$, contaminating the crystalline emission at this wavelength. SAGE 050830 has a very high peak-to-continuum ratio of 5.05 (with a $11.3 / 9.8 \mu \mathrm{m}$ ratio of 0.81 ), and falls outside our plot range. Most of our sources show rather high $11.3 / 9.8 \mu \mathrm{m}$ ratios, with low peak-tocontinuum values, showing that the crystallinity fraction is high, and the average grain sizes relatively large.

A very weak correlation (Kendall rank correlation $\tau=$ $-0.23)$, can be seen. This is in contrast to the strong correlation seen in discs around young stars between grain growth and crystallisation processes (van Boekel et al. 2003, 2005; Juhász et al. 2010). The gray area in Fig. 4 shows typical values found for protoplanetary discs, and it is clear that our sources show a much larger spread in values for the continuum-subtracted $11.3 / 9.8 \mu \mathrm{m}$ flux ratio. This could mean that in the case of the post-AGB discs, the dust might not consist of very small 


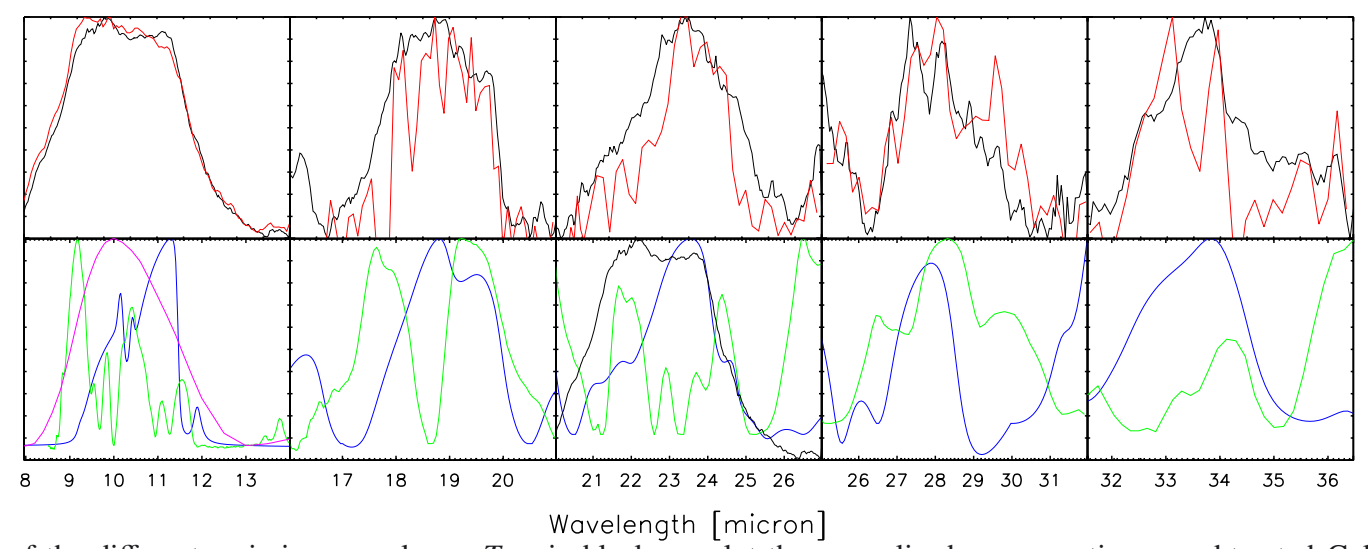

Fig. 3. Overview of the different emission complexes. Top: in black we plot the normalised mean continuum-subtracted Galactic spectrum, in red the normalised mean LMC spectrum. We did not include the 14 and $16 \mu \mathrm{m}$ complexes, since the noise level for the LMC sources made it impossible to determine a mean spectrum. Bottom: the different normalised continuum-subtracted spectra of forsterite, enstatite and amorphous olivine are given in respectively blue, green and magenta.

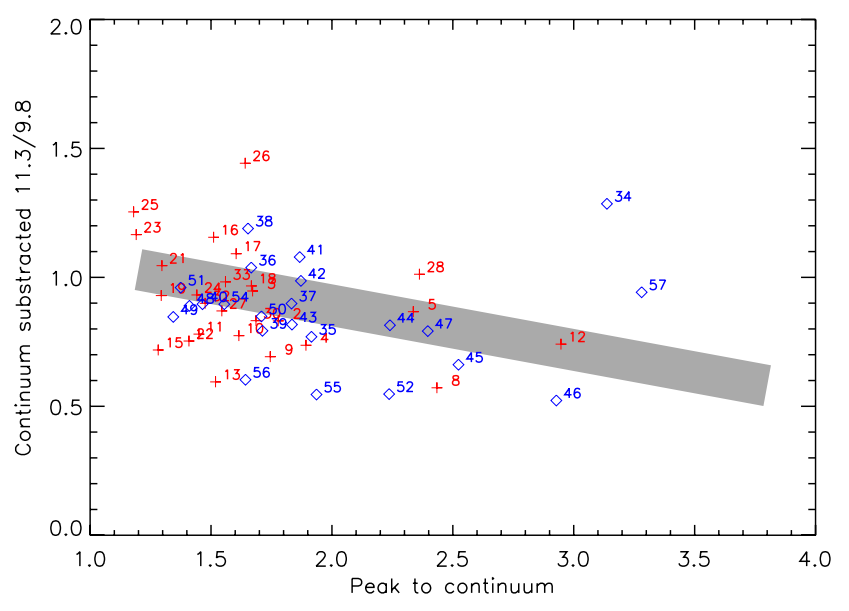

Fig. 4. Ratio of the continuum-subtracted flux at 9.8 and $11.3 \mu \mathrm{m}$ versus the peak-to-continuum ratio of the $10 \mu \mathrm{m}$ silicate feature. Galactic sources are plotted in red plus signs, LMC sources in blue diamonds. The gray area shows typical values found for protoplanetary discs around young stars. The numbers correspond to numbers given in Tables 1 and 2 .

$(0.1 \mu \mathrm{m})$ amorphous grains, but may already have a higher crystallinity or larger grain size. It could also mean that different grain processes are at play, resulting in a slightly different dust grain evolution. We find no evidence for different behaviour between our Galactic and LMC samples.

\subsection{The $14 \mu \mathrm{m}$ and $16 \mu \mathrm{m}$ complexes $(13.5-15 \mu \mathrm{m}$ and $15-17 \mu \mathrm{m})$}

This region is dominated by two different emission complexes, respectively around 14 and $16 \mu \mathrm{m}$, as can be seen in Fig. A.1. Because of the high noise level in the LMC sources for this region, we could only calculate a mean spectrum for the Galactic stars.

The $14 \mu \mathrm{m}$ complex is sensitive to the emission of enstatite, which shows a clear feature around $13.8 \mu \mathrm{m}$ in our observed sources. The predicted feature at $14.4 \mu \mathrm{m}$, however, is not seen. Instead, we do see a clear signature around $14.7 \mu \mathrm{m}$. The synthetic spectra of enstatite are known to be sensitive to the refractory indices used and the adopted grain size (see for example
Fig. 10 in Molster et al. 2002a, and Fig. 20 in Juhász et al. 2010). Chihara et al. (2002) present an overview of the shift of peak position of crystalline pyroxenes with different iron contributions and we find that the peak positions found in our spectra are better modelled with enstatite with a small iron contribution of about $10 \%$. In Fig. 6 we show the continuum-subtracted spectrum of ST Pup, which has the strongest enstatite features and best $S / N$ ratio in this region of our sample stars, together with the laboratory spectra of ortho-enstatite and clino-enstatite with a $10 \%$ iron content, as presented by Chihara et al. (2002). Unfortunately, the $14 \mu \mathrm{m}$ complex is the only wavelength region where the enstatite features are not blended with forsterite emission. This makes it impossible to study the enstatite iron content using other complexes.

In the Galactic sample the $16 \mu \mathrm{m}$ complex is clearly visible in most sources, the outliers being EP Lyr and IRAS 10174. The feature seen in IRAS 15556 is strongly deviating from the mean complex, and shows a stronger contribution of enstatite emission. IRAS 06338 shows strong emission of $\mathrm{CO}_{2}$ gas in the $16 \mu \mathrm{m}$ region.

As was already discussed in Gielen et al. (2008), the strength of the $16 \mu \mathrm{m}$ feature seems to correspond to the emission of forsterite, shifted bluewards in central wavelength. Our new spectra follow this trend. This shift of the $16 \mu \mathrm{m}$ feature is also seen in the infrared spectra of protoplanetary discs (Juhász et al. 2010), and is probably an effect of the adopted synthetic spectrum of forsterite.

\subsection{The $19 \mu \mathrm{m}$ and $23 \mu \mathrm{m}$ complexes (17-21 $\mu \mathrm{m}$ and $21-26 \mu \mathrm{m}$ )}

This region shows two strong emission complexes, around 19 and $23 \mu \mathrm{m}$, and a good agreement between the two samples is found (see Figs. A.2 and A.3). Although the LMC sample has strong noise, the mean spectrum is very similar to the Galactic mean.

The $19 \mu \mathrm{m}$ feature seems to be more pronounced in the Galactic sources, which could mean that the LMC sample is less crystalline, since the feature is mainly formed by forsterite emission. However, the strong noise level of the LMC sources could also hamper the detection of the feature. The $23 \mu \mathrm{m}$ feature is dominated by the emission of forsterite, and is clearly seen in most Galactic and several LMC stars. The mean Galactic and LMC complexes are again very similar. In the 

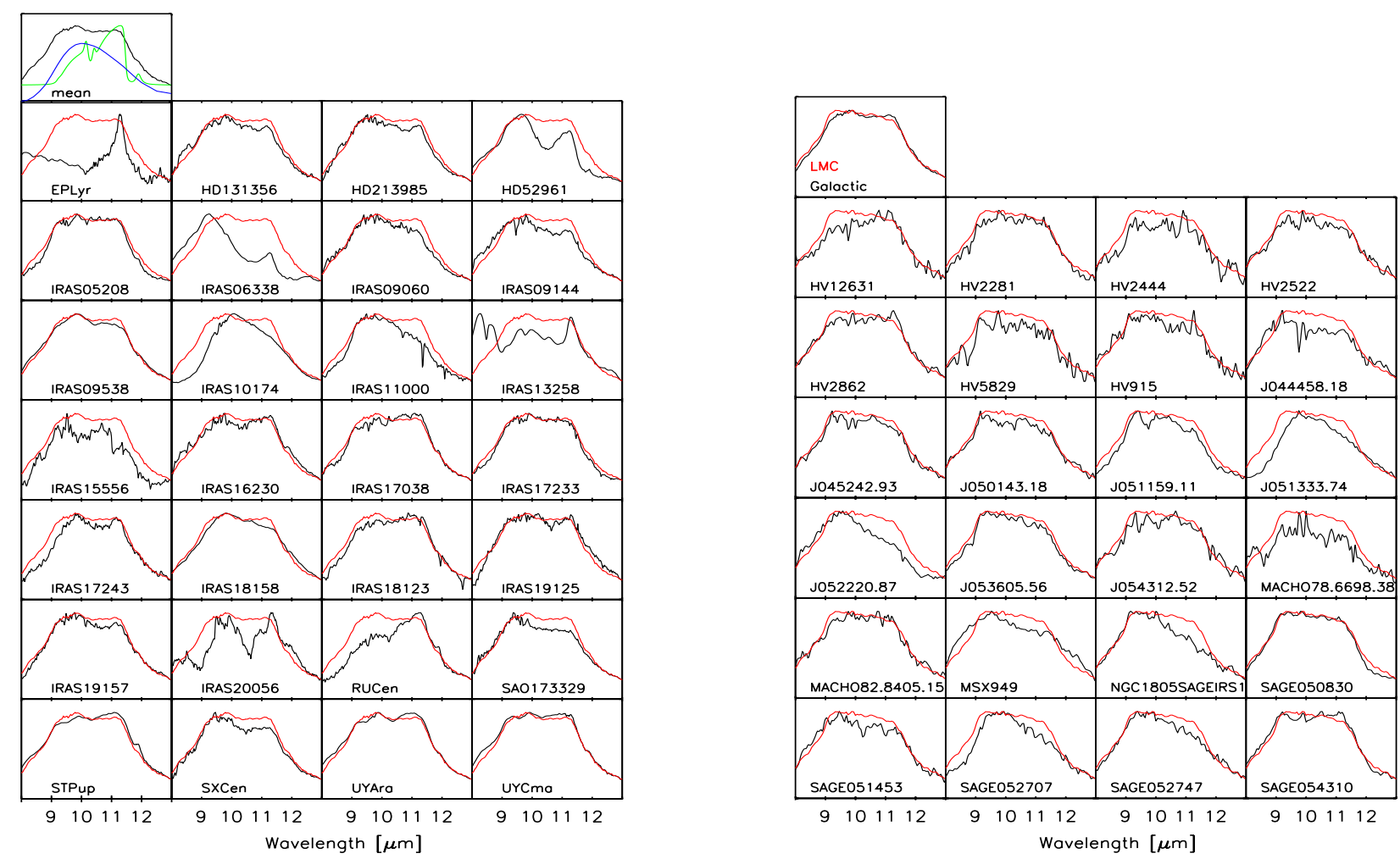

Fig. 5. Left: the $10 \mu \mathrm{m}$ complex for the Galactic sources, continuum subtracted and normalised. Overplotted in red the mean spectrum. The mass absorption coefficients of amorphous olivine and forsterite are plotted in blue and green. Right: same as on the left, but for the LMC sources. The top panel shows the comparison between the calculated means for the LMC and Galactic sources.

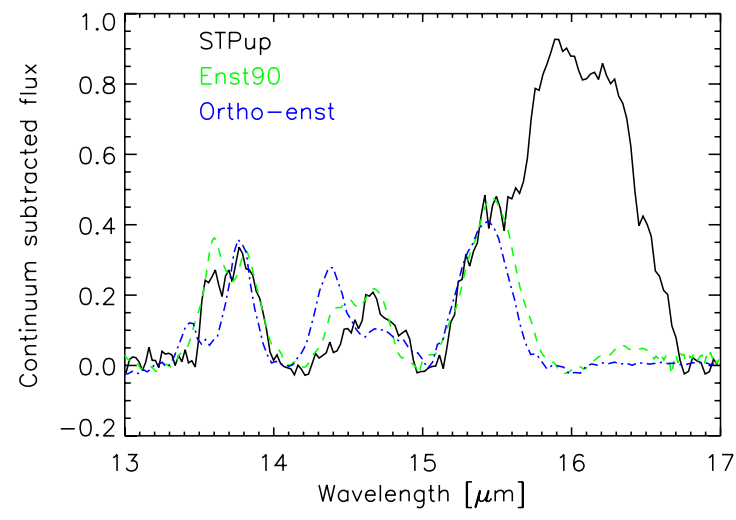

Fig. 6. Comparison between the normalised and continuum-subtracted spectrum of one of our sample stars ST Pup and the laboratory spectra of ortho-enstatite (dot-dashed line) and clino-enstatite (dashed line) with a $10 \%$ iron content, as presented by Chihara et al. (2002). The strong observed feature in ST Pup around $16 \mu \mathrm{m}$ is due to forsterite.

Galactic sources, the same outliers appear again: EPLyr and HD 52961, which have a very particular mineralogy (Gielen et al. 2009b); IRAS 10174, which is almost completely amorphous, and IRAS 15556 which shows no emission at $23 \mu \mathrm{m}$.

\subsection{The $27 \mu \mathrm{m}$ and $33 \mu \mathrm{m}$ complexes $(25.5-30 \mu \mathrm{m}$ and $32-36 \mu \mathrm{m}$ )}

The two samples show a similar observed mean features, peaking around 27 and $33 \mu \mathrm{m}$ (see Figs. A.4 and A.5).
The $27 \mu \mathrm{m}$ complex peaks at the forsterite $27.5 \mu \mathrm{m}$ feature, but with an additional shoulder around $29.3 \mu \mathrm{m}$, which is due to enstatite emission. In the Galactic sample, strong deviation can again be seen for IRAS 06338, which is clearly a source with atypical dust emission features. Some sources show a somewhat broader $27 \mu \mathrm{m}$ feature, such as IRAS 15556, which could point to a larger enstatite contribution. The LMC sample is again compromised by the strong noise, but the stars with strong observed emission feature do show a similar feature as observed in the Galactic sample. Only MSX 949 seems to deviate from the observed mean complex, with a very broad feature which peaks at $29.5 \mu \mathrm{m}$.

Both samples show a clear $33 \mu \mathrm{m}$ feature, due to the emission of forsterite crystals at lower temperature. For the LMC sample the spectrum around the $33 \mu \mathrm{m}$ complex is strongly hampered by high noise, but the feature is still visible in the mean spectrum. A few sources have very strong emission at $33 \mu \mathrm{m}$, such as HV 12631, J044458, MACHO 78.6698.38, MSX 949, SAGE 054310, and SAGE 050830.

\section{Full spectral model}

To study the characteristics of the silicate emission observed in these sources, we constructed a basic model to fit the full Spitzer wavelength range. The observed emission features will depend on the chemical composition of the dust, the grain sizes and the grain shapes. In Gielen et al. (2008) we constructed a model that takes all the above properties into account. Note that a bug was present in the modelling routine used in Gielen et al. (2008, 2009a), which we describe in Gielen et al. (2010). For this paper, we also extended the routine to include an additional dust species, namely amorphous silica $\left(\mathrm{SiO}_{2}\right)$. 
Table 3. Overview of the adopted dust species.

\begin{tabular}{lcccccc}
\hline \hline Dust species & Composition & Structure & Density & Shape & Grain size & Reference \\
\hline Olivine & $\mathrm{Mg}_{2} \mathrm{SiO}_{4}$ & $\mathrm{~A}$ & $3.71 \mathrm{~g} / \mathrm{cm}^{3}$ & $\mathrm{GRF}$ & $0.1-2-4 \mu \mathrm{m}$ & Dorschner et al. (1995) \\
Pyroxene & $\mathrm{MgSiO}_{3}$ & $\mathrm{~A}$ & $3.20 \mathrm{~g} / \mathrm{cm}^{3}$ & $\mathrm{GRF}$ & $0.1-2-4 \mu \mathrm{m}$ & Dorschner et al. (1995) \\
Olivine & $\mathrm{MgFeSiO}_{4}$ & $\mathrm{~A}$ & $3.71 \mathrm{~g} / \mathrm{cm}^{3}$ & $\mathrm{GRF}$ & $0.1-2-4 \mu \mathrm{m}$ & Dorschner et al. (1995) \\
Pyroxene & $\mathrm{MgFeSi}_{2} \mathrm{O}_{6}$ & $\mathrm{~A}$ & $3.20 \mathrm{~g} / \mathrm{cm}^{3}$ & $\mathrm{GRF}$ & $0.1-2-4 \mu \mathrm{m}$ & Dorschner et al. (1995) \\
Forsterite & $\mathrm{Mg}_{2} \mathrm{SiO}_{4}$ & $\mathrm{C}$ & $3.33 \mathrm{~g} / \mathrm{cm}^{3}$ & $\mathrm{GRF}$ & $0.1-2-4 \mu \mathrm{m}$ & Servoin \& Pirou (1973) \\
Ortho-Enstatite & $\mathrm{MgSiO}_{3}$ & $\mathrm{C}$ & $2.80 \mathrm{~g} / \mathrm{cm}^{3}$ & $\mathrm{GRF}$ & $0.1-2-4 \mu \mathrm{m}$ & Jaeger et al. (1998) \\
Silica & $\mathrm{SiO}_{2}$ & A & $2.20 \mathrm{~g} / \mathrm{cm}^{3}$ & GRF & $0.1-2-4 \mu \mathrm{m}$ & Henning \& Mutschke (1997) \\
\hline
\end{tabular}

Notes. For each component we list its chemical composition, whether it has an amorphous (A) or crystalline (C) structure, density, adopted grain shape and grain sizes, and reference to the refractory indices used.

Assuming that the dust features are formed in an optically thin upper part of the disc, the spectrum can be approximated as a linear combination of dust absorption profiles. The model emission is then given by

$F_{\lambda} \sim\left(\sum_{i} \alpha_{i} \kappa_{i}\right) \times\left(\sum_{j} \beta_{j} B_{\lambda}\left(T_{j}\right)\right)+F_{\mathrm{cont}}$

where $\kappa_{i}$ is the mass absorption coefficient of dust component $i$ and $\alpha_{i}$ gives the fraction of that dust component, $B_{\lambda}\left(T_{j}\right)$ denotes the Planck function at temperature $T_{j}$ and $\beta_{j}$ a scaling factor for the Planck functions. A sum of two Planck functions is also used to represent the continuum flux $F_{\text {cont }}$. Following Gielen et al. (2008), we use two different dust and continuum temperatures, ranging from $100 \mathrm{~K}$ to $1000 \mathrm{~K}$.

The dust species we included are amorphous olivine/pyroxene $\left(\mathrm{Mg}_{2 x} \mathrm{Fe}_{2(1-x)} \mathrm{SiO}_{4} / \mathrm{Mg}_{x} \mathrm{Fe}_{1-x} \mathrm{SiO}_{3}\right)$, crystalline olivine/ pyroxene (forsterite/enstatite) and amorphous silica. Silica has different polymorphs, such as quartz, cristobalite and tridimite, with similar emission profiles (e.g. Sargent et al. 2009), and we cannot rule out that some of these other polymorphs contribute to the silica fraction. To keep the number of free parameters to a minimum, we opted to use only amorphous silica in our modelling. In Sect. 4.2 we showed that (part of) the enstatite content in our discs might be in the form of clino-enstatite with a $10 \%$ iron content. Unfortunately, the laboratory data of this enstatite species does not allow to calculate synthetic spectra for different grain sizes, so we opted to use the more commonly used ironfree ortho-enstatite. As discussed above (Sect. 4), our study of the different complexes show that these dust species are present, and that there is no strong evidence for the presence of other dust species.

Mass absorption coefficients for the different dust species are calculated from refractory indices in Gaussian random fields (GRF) dust approximation (Shkuratov \& Grynko 2005). The details of the different refractory indices that we used can be found in Table 3. From our previous spectral studies we know that the observed emission features are reproduced using a non-spherical grain shape. Even though the continuous distribution of ellipsoids approximation (CDE, Bohren et al. 1983) is widely used, it is unfortunately only valid in the Rayleigh limit, and does not allow us to study grain growth effects. For this reason we prefer the GRF approximation. We also tested the distribution of hollow spheres approximation (DHS, Min et al. 2005), but this did not result in a better fit to the observed emission features.

To study the grain size distribution inferred from the modelling, we use three discrete dust grain sizes in the model: 0.1 , 2.0 and $4.0 \mu \mathrm{m}$. The emission features of grains with larger sizes become too weak to distinguish from the continuum emission. In Gielen et al. (2008) we already found that the presence of
Mg-rich amorphous grains cannot be ruled out, and thus here also we use both purely Mg-rich amorphous silicates $(x=1)$ and amorphous silicates with an equal amount of $\mathrm{Mg}$ and $\mathrm{Fe}$ $(x=0.5)$. The ratio of magnesium and iron in the amorphous silicates mainly changes the peak position of the 10 and $18 \mu \mathrm{m}$ emission features (Dorschner et al. 1995).

The best model was calculated using standard $\chi^{2}$ minimalisation. Errors on the model parameters were calculated using a 100 step Monte Carlo simulation with Gaussian noise distribution. Even though this model is only a first approximation, the model clearly succeeds in giving an overall good fit to the observed spectra (see Figs. A.6, A.7).

For 6 sample (Galactic) sources the Spitzer spectrum only starts at $9.9 \mu \mathrm{m}$, which means we lack information on the dust composition in the $10 \mu \mathrm{m}$ wavelength range. Since this could influence the derived dust parameters, we depict these sources in a different color in our correlation plots (Figs. 7-A.9).

\subsection{Results}

We find that, for most sources, the dust is dominated by large grains. We define the mass-weighted mean grain size of the dust as

$a_{\text {mean }}=\Sigma m_{i} a_{i}$,

with $a_{i}$ the grain size, and $m_{i}$ the mass fraction of dust in that grain size. For $47 / 57$ of our sample stars, the mean grain size is larger than $2 \mu \mathrm{m}$ (see Fig. 7).

Since our model routine uses three different grain sizes, we can use them to determine a grain size distribution. The Spitzer spectra probably only trace the upper layers of the disc, and so the calculated distribution could not be valid for the entire disc. The grain size distribution is usually approximated by $n(a) \propto$ $a^{p}$, with $n(a)$ the number of grains with grain size $a$, and $p$ a power-law index. For the interstellar medium a value of $p=$ -3.5 is found (Mathis et al. 1977) for typical ISM grains up to $0.3 \mu \mathrm{m}$, rolling over exponentially for larger grains (Zubko et al. 2004). To calculate the number of grains in a given grain size, we compute the mass fraction of these grains from our modelling and divide it by the corresponding volume of the grains. We then normalise all the grain numbers, such that $n(0.1 \mu \mathrm{m})=1$. The results of this calculation can be seen in Fig. 8. We find a good fit to our results is achieved with a power-law index $p=-1.3_{0.2}^{0.1}$, for grain sizes between 0.1 and $4 \mu \mathrm{m}$. It is clear that our grain size distribution is not ISM like, larger grains are much more abundant.

From Fig. A.9 it is clear that for about half the stars the crystalline grains are larger than the amorphous grains. For nearly all stars the mean grain size of the crystalline grains lies above 


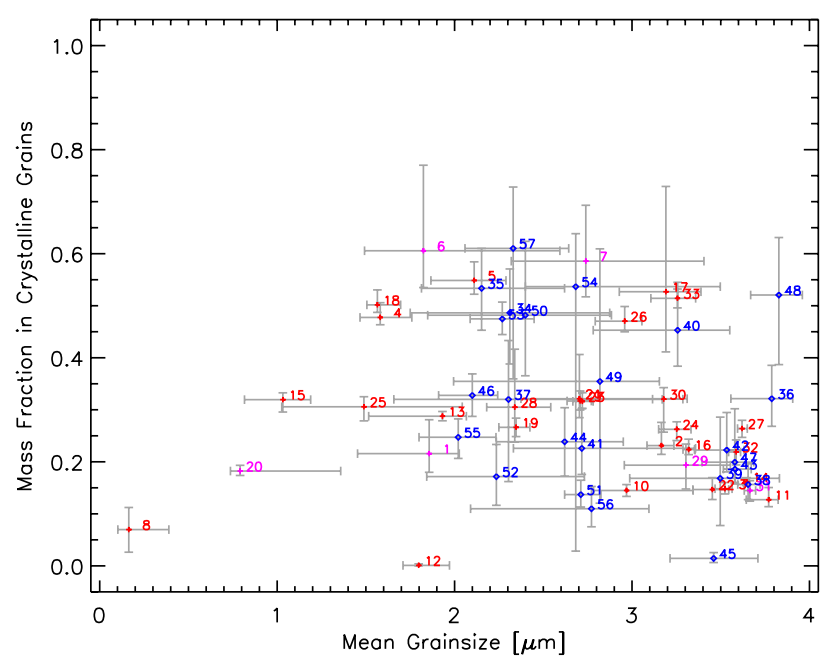

Fig. 7. The mass fraction in crystalline grains versus the mean grain size of our spectral modelling. Galactic sources are given in red plus signs and LMC sources in blue diamonds. The magenta symbols depict Galactic sources for which the infrared spectra only start from $9.9 \mu \mathrm{m}$. The numbers correspond to numbers given in Tables 1 and 2 .

$2 \mu \mathrm{m}$, whereas the amorphous grains show a larger spread in grain sizes. This is in contrast to what is found for the dust in discs around Herbig Ae stars, where the crystalline grains are significantly smaller than the amorphous grains (Juhász et al. 2010). We do not find any correlation between the size of crystalline and amorphous material. It is unclear what causes this difference in grain size between the amorphous and crystalline dust. An effect that could come into play here is the apparent spectral signature of large dust aggregates. Min et al. (2008) showed that aggregates with a very low abundance appear spectroscopically as very small grains, while more abundant materials appear spectroscopically to reside in larger grains. Since for our sources the amorphous dust is in most cases more abundant than the crystalline dust, this could mean that the amorphous grains reside in large fluffy aggregates, which have spectral signatures that are very similar to those of small grains (Min et al. 2006, 2008).

Similar to what is found in Juhász et al. (2010) we find that the size of the enstatite grains is on average slightly larger than that of the forsterite grains. There seems to be a weak trend between the crystallinity and the mean size of the crystalline grains: sources with a higher crystallinity have on average larger crystalline grain sizes (see Fig. A.9).

For discs around young stars a strong correlation is found between the mean grain size of the amorphous grains and the disc flaring. This disc flaring is determined by the ratio of the $24 \mu \mathrm{m}$ and $8 \mu \mathrm{m}$ flux. Juhász et al. (2010) find that sources with flatter discs have larger amorphous grains in their disc atmosphere. This trend is not seen in our sample. Our sample sources seem to be centred around $F_{24} / F_{8}=0.83$, which shows that these discs are not strongly flared and that there is no large spread in disc flaring. Of the 57 sources, 8 show higher values of the disc flaring, going from $F_{24} / F_{8}=2$ up to 4.3 . We find no correlation between the disc flaring and any other dust parameter.

Figure 7 also shows the high crystallinity fraction derived from the Spitzer modelling. The crystallinity can reach values of $60 \%$, which is among the highest seen in astronomical environments. High values of crystalline dust are also found for protoplanetary discs around young stars (e.g. Bouwman et al. 2008; Juhász et al. 2010), where crystalline fractions up to about $30 \%$

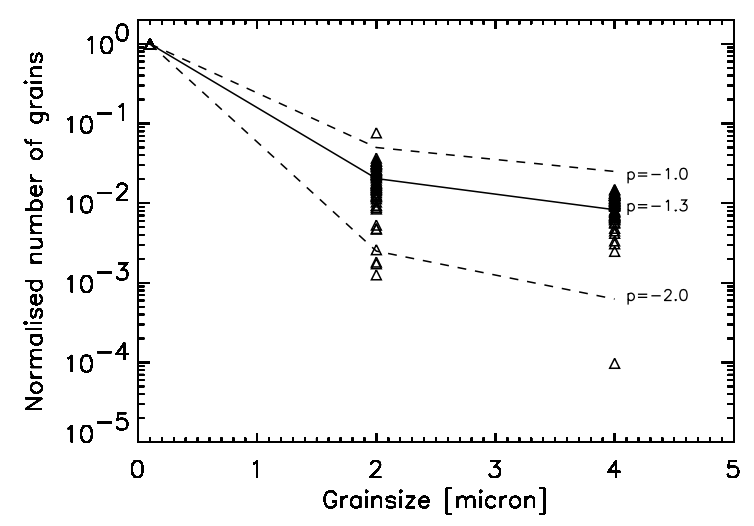

Fig. 8. The normalised number of grains versus the adopted grain sizes. The triangles represent all the sample stars. The solid line gives the best power-law distribution to the mean of all stars. The dashed lines represent different power-law indices, given for comparison.

are found. For the crystalline dust, forsterite is almost always the dominant species: the forsterite fraction of the crystalline material has values between 20 and 100\% (Fig. A.11). There does not seem to be a strong correlation between the crystallinity and the forsterite/enstatite fraction of the crystalline material. The same holds for the forsterite/enstatite fraction of the crystalline material and the mean grain size of the crystalline grains (see Fig. A.11).

The derived silica fractions are of the order of 5\%, but can go up to $20 \%$. We find no correlation between the silica mass fraction and any other dust parameters. Thermal annealing of amorphous dust produces both forsterite and silica, and so a relation is to be expected if this process is responsible for the crystallisation.

In our modelling we used amorphous silicates with an equal $\mathrm{Mg}$-Fe content and pure $\mathrm{Mg}$-rich grains. We find that $25 / 57$ sources show a dominance of iron-free amorphous dust, whereas the other sources are clearly dominated by Mg-Fe amorphous silicates. On average we find that the Galactic sources have a slightly higher fraction $(53 \%)$ of the $\mathrm{Mg}-\mathrm{Fe}$ rich amorphous silicates, whereas the LMC sources have a higher fraction (56\%) of purely Mg-rich amorphous silicates. The derived differences are very minimal, so we cannot make strong statements on the iron content of the amorphous silicates. For the sources where we find a high fraction of iron-free dust, the iron grains could be stored as metallic inclusions in the grains, which would be very hard to detect.

We do not find any correlation between the derived dust parameters and central binary parameters such as the effective temperature or the orbit.

\subsection{Atypical sources: intruders?}

Some sources clearly deviate from the mean observed spectrum, by showing no crystalline grains (IRAS 10174 and J 05133) or carbonaceous molecules and/or gas emission (EPLyr, HD 52961, IRAS 06338 and IRAS 13258). Other sources, such as MSX 949, show less obvious differences, but are still not reproduced as well by the model as the other sources. Since these sources were mainly selected on the basis of their infrared colours, we cannot exclude that non-post-AGB disc sources are present in the sample. Possible intruders could be young stars with protoplanetary discs, red super giants or AGB stars. 
However, for most sources we have additional observations of the central star, such as optical spectroscopy, which corroborate their post-AGB evolutionary phase. We discuss several doubtful (or anomalous) cases below.

\subsubsection{SAGE 050830}

For SAGE 050830, the optical spectra show some evidence for a carbon-rich chemistry (van Aarle et al. 2011). However, the photometry and infrared spectral information for this source is suspected to be contaminated by a foreground star of spectral type A0-1IV. Unfortunately, the angular resolution does not allow us to discriminate between the A star and the carbon star as the identification of the Spitzer source. Still, if the carbon-rich spectrum truly belongs to the Spitzer source, the strong oxygenrich spectrum seems surprising. This source is one of the more crystalline objects of our sample, and even has the most extreme $10 \mu \mathrm{m}$ feature-to-continuum ratio of all sources!

The carbon-rich classification of the central star, together with the presence of crystalline silicates in its circumstellar environment would make this star an ideal candidate to be a silicate J-type carbon star. These are carbon-rich AGB stars, but with a very low ${ }^{12} \mathrm{C} /{ }^{13} \mathrm{C}$ ratio and detection of crystalline silicates in their infrared spectrum (Lloyd Evans 1990; Abia \& Isern 2000). The sources are believed to be binary stars, with an unseen companion, surrounded by a circumbinary disc (Morris 1990; Jura \& Kahane 1999; Yamamura et al. 2000; Deroo et al. 2007). This scenario could explain the dual chemistry, since the disc could then be formed while the central star was still oxygen rich, and has now evolved to be carbon rich. However, it does not explain the low ${ }^{12} \mathrm{C} /{ }^{13} \mathrm{C}$ ratio, usually seen in J-type silicate carbon stars.

Unfortunately, due to the confusion with the foreground star, we cannot determine the stellar parameters, which would shed light on the evolutionary status of this object. Also, the lowresolution optical spectrum does not allow to determine the ${ }^{12} \mathrm{C} /{ }^{13} \mathrm{C}$ ratio of the carbon star, hence corroborating the J-type nature.

\subsubsection{J 05133 and IRAS 10174}

Two of our sample sources, IRAS 10174 and J 05133, clearly deviate from the rest of the sample by showing no strong evidence for crystalline features in their spectra. However, our modelling shows that purely amorphous silicate dust is not sufficient in reproducing the observed features, especially around $13 \mu \mathrm{m}$. The spectra of these stars are actually very similar to the observed spectra of AGB outflows, characterised by small amorphous grains. In these sources, emission from additional dust species, such as alumina $\left(\mathrm{Al}_{2} \mathrm{O}_{3}\right)$, can influence the $13 \mu \mathrm{m}$ region. To see if alumina could also be present in these sources, we remodelled the spectra, now including alumina grains. For both stars we find an improvement when including $\mathrm{Al}_{2} \mathrm{O}_{3}$, especially for J 05133 . For IRAS 10174 the improvement is only minor, with an amount of alumina in the new model of $2 \%$. However, for J 05133 the fit is improved drastically when including 30\% alumina (Fig. 9). The bulk of the other dust ( 50\%) is stored in small $0.1 \mu \mathrm{m} \mathrm{Mg-rich} \mathrm{olivine} \mathrm{in} \mathrm{this} \mathrm{new} \mathrm{model.} \mathrm{Less}$ then $10 \%$ of the mass fraction of dust is in crystalline form, and then mainly forsterite. This type of dust composition is more indicative of an outflow and not a disc.

The optical spectrum of J05133 (van Aarle et al. 2011) also indicates the peculiar nature of this source. The spectrum points to a F8-G0Ip spectral classification, but shows very strong

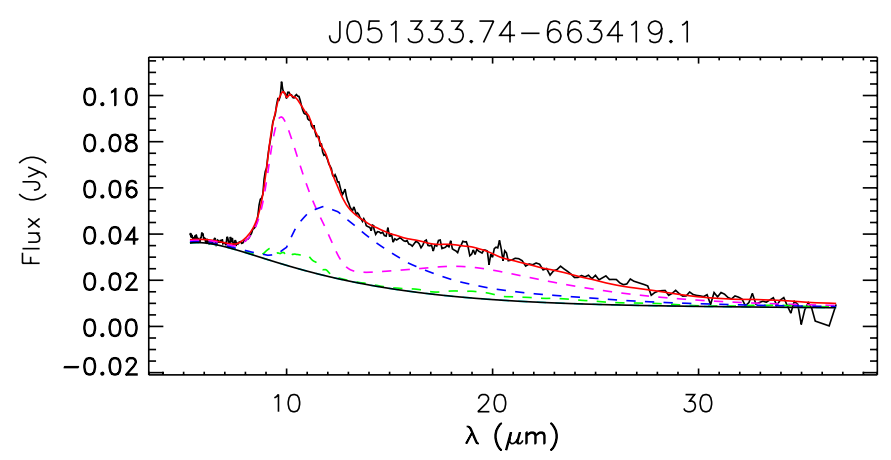

Fig. 9. The results of our modelling of $\mathrm{J} 05133.74$, with the addition of alumina grains. The observed spectrum (black curve) is plotted together with the best model fit (red curve) and the continuum (black solid line). Crystalline silicates are plotted in green, amorphous silicates in magenta and alumina in blue.

H I (6563 ̊) and He I (5876 $)$ emission, and broad Ca II absorption lines, which are not expected in a star of this type, but point to the presence of a hotter source. One possibility would be that the system is actually a binary with an unseen hot companion. The SED modelling gives, for this source, a luminosity of around $17000 L_{\odot}$. This, combined with the spectral type as derived from the optical spectrum, shows that the source cannot be an AGB star, but also shows that it is probably not a postAGB disc source as normally understood.

The unusual chemistry of IRAS 10174 compared to the rest of the sample made us re-investigate the optical spectra for this source. The source was originally classified as a post-AGB source (Lloyd Evans 1999; De Ruyter et al. 2006) from a blue spectrum which suffered badly from dust extinction in the violet. A new optical spectrum (6400-9000 ̊), taken with the Cassegrain grating spectrograph of the $1.9 \mathrm{~m}$ Radcliffe Telescope at the South African Astronomical Observatory, shows that the star is a luminous supergiant, of type G8Ia-O. This is in better agreement with the observed chemistry of the infrared spectrum. Note that even though the star is not a postAGB disc source, the binarity of the star is confirmed (Maas et al. 2003).

Even though some non-post-AGB disc sources might be present in the sample, these sources do not change the overall conclusions of this study. The removal of these sources does not introduce correlations between the different stellar and dust parameters, that are currently not observed.

\section{Discussion}

For all sources, the infrared spectra are dominated by emission features due to oxygen-rich dust species. For some of the LMC sources (such as J051159.11, J053605.56, and SAGE 054310) this is surprising, since they have luminosities which would put them in the peak of the carbon star luminosity function (Stancliffe et al. 2005; Groenewegen et al. 2007; Srinivasan et al. 2011). Optical spectroscopy for these sources does not point to a carbon-rich chemistry of the central star (van Aarle et al. 2011). This shows that the AGB evolution for these binary post-AGB stars was shortcut, possibly under the influence of strong binary interaction, preventing them to evolve into carbon stars.

Our study shows that even a relatively simple model succeeds in reproducing the observed infrared spectra. The model assumes only two dust temperatures, and uses the same dust 
abundances for the cool and warm dust. This shows that the dust in the disc is relatively well mixed, in that cooler and hotter regions in the disc have a similar dust composition. This is very different from the results for protoplanetary discs around young stars, where a difference in dust composition is needed for the inner and outer disc regions (Juhász et al. 2010).

The strong observed crystalline bands at longer wavelengths show that at least a significant fraction of the crystalline grains are located at cooler temperatures. For some sources our model even underestimates the forsterite flux at $33 \mu \mathrm{m}$, showing that, for some sources at least, the forsterite fraction between the two temperatures might not be evenly distributed, but dominated by the cooler temperature. The problem of reproducing the features at longer wavelengths might also be due to the adopted synthetic spectra of forsterite. As can be seen in Fig. 3.14 of Gielen et al. (2008), the $33 \mu \mathrm{m}$ is best reproduced by DHS grain shapes. The GRF grain shape gives a feature which is much broader and flat topped. However, since on average our features were slightly better reproduced with GRF shapes, we used this approximation in our modelling. Another effect that can influence the observed features in the optical depth. At different wavelengths we would look at different depths in the disc, with a different temperature distribution. At longer wavelengths, we would then look deeper in the disc, where the cooler temperature might enhance the features at these longer wavelengths. To study this effect in detail, a full radiative transfer model is needed, which goes beyond the scope of this paper.

Since the exact formation mechanism of these circumbinary discs is still uncertain, it is difficult the relate the different observed dust characteristics to disc evolution. One possibility is that the discs are formed after a common-envelope phase, with some dust formation already forming in the outflow phase. Crystalline grains can then be formed directly out of the gas phase, at high temperatures (Gail 2004; Petaev \& Wood 2005). But this will probably not give rise to the very high amounts of crystalline material we see, which shows that another crystallisation process is still active afterwards, such as thermal annealing (Wooden et al. 2005). Another formation mechanism is RocheLobe overflow through an outer Langrangian point, where the material is already confined to the midplane. Here one could expect the dust at the hot and denser inner regions to be more efficient in producing crystalline species. Both gas-phase condensation and thermal annealing might be important to explain the high crystallinity in these discs.

The condensation models predict forsterite to condense first, followed by the formation of enstatite through reactions between forsterite and $\mathrm{SiO}_{2}$ gas. In contrast to what is found for protoplanetary discs, our results show that forsterite is almost always the dominant crystalline dust species. This could point to a deviation from equilibrium conditions during condensation. If the forsterite grains reach large grain sizes quickly, the formation of enstatite might be complicated, since it will become increasingly harder to infuse $\mathrm{SiO}_{2}$ in the forserite lattice. The resulting dust may the be in the form of a large forsterite grain, surrounded by a small layer of enstatite. The formation of enstatite can be further weakened if the material is allowed to cool very quickly after the condensation of forsterite. The formation of forsterite through annealing is especially efficient if the starting material has an olivine stoichiometry. A high forsterite fraction would then go together with a higher pyroxene fraction of the amorphous material, which is not supported by our results.

Since crystallisation requires high temperatures above $1000 \mathrm{~K}$ (Fabian et al. 2000), one would expect the crystalline dust to be confined to the hot, inner regions of the disc. This is in clear contrast to our findings of cool crystalline material and a homogeneous dust composition throughout the disc. This shows that mixing must be efficient is transporting the crystalline material to cooler regions which were initially dominated by amorphous material, or a crystallisation process at lower temperatures is occurring in the discs. Molster et al. (2002a,b) already showed that the crystallinity fraction in disc sources is much higher than that observed in typical outflow sources. This shows that the crystalline component in the disc sources is most likely determined by subsequent dust grain processing in the discs, and not by cooling processes in the outflow of the material forming the discs.

Dust formation models also show that iron will preferably condense out as metallic iron, rather than be included in silicate formation. This could explain the presence of Mg-rich amorphous silicates in our results. The grain sizes of the crystalline dust, formed through condensation, will not be correlated with the grain sizes of the amorphous material, which is in line with our results. If the crystallisation occurs through annealing, we would expect a relation between the initial amorphous material and final crystalline grains. This does not explain the observed difference in crystalline and amorphous grain sizes, unless a subsequent process can be invoked that would grow the crystalline material, but not the amorphous dust. Our results on the difference in crystalline and amorphous grain sizes is again in clear contrast to what is found for the dust in protoplanetary discs, where the crystalline grains are found to be significantly smaller than the amorphous grains (Juhász et al. 2010). Clearly, different dust processes are responsible for the grain growth and crystallisation in the discs around young and evolved stars.

The derived large grain sizes show that there seems to be an efficient removal of the smallest grains. The question remains whether this lack of small grains is an effect of grain growth (Dullemond \& Dominik 2004) or whether the initial grain population already consisted of large grains. In that case the smallgrain fraction could be a result of grain collision and subsequent break-up. An effect which might also be important to the observed grain sizes is the strong radiation of the central source. The central post-AGB stars are highly luminous, and radiation pressure could be responsible for the removal of the smallest grains in the upper layers of the disc. Since our results show that the amorphous grains tend to be smaller than the crystalline grains, radiation pressure might be (partly) responsible for the large fraction of crystalline grains observed in the upper layers of the disc.

Surprisingly, we find no correlations between the derived dust parameters, such as crystallinity, grain size and abundances. Also, no correlation between the dust parameters and parameters of the central binary system is found. The lack of correlation raises the question whether the optically thin upper layers traced by the Spitzer spectra are a good representative of the global dust composition.

Except for the amorphous silicate dust, we find no evidence for the presence of dust species usually associated with AGB outflows or single-star post-AGB shells, such as simple oxides or $\mathrm{Al} / \mathrm{Ca}$-bearing dust species. The theoretical oxygenrich dust condensation sequence for dusty outflows starts with the formation of alumina $\left(\mathrm{Al}_{2} \mathrm{O}_{3}\right)$ around $1760 \mathrm{~K}$, followed by formation of gehlenite $\left(\mathrm{Ca}_{2} \mathrm{Al}_{2} \mathrm{SiO}_{7}\right)$ at slightly lower temperatures (Tielens 1990; Tielens et al. 1998). Further interactions with magnesium will produce species like spinel $\left(\mathrm{MgAl}_{2} \mathrm{O}_{4}\right)$, akermanite $\left(\mathrm{Ca}_{2} \mathrm{MgSi}_{2} \mathrm{O}_{7}\right)$, diopside $\left(\mathrm{CaMgSi}_{2} \mathrm{O}_{6}\right)$ and finally anorthite $\left(\mathrm{Ca}_{2} \mathrm{Al}_{2} \mathrm{Si}_{2} \mathrm{O}_{8}\right)$ around $1360 \mathrm{~K}$. A second condensation sequence, involving mostly magnesium and silicon, starts with 
the formation of forsterite around $1500 \mathrm{~K}$, followed by enstatite around $1300 \mathrm{~K}$. Only at temperatures below the glass temperature can iron interact to form amorphous iron-containing silicates. Not only the temperature plays a role, also the densities involved will determine which dust species can be formed.

From observations it is found that AGB stars start by forming $\mathrm{Al}-$ and $\mathrm{Mg}$-rich oxides in their outflows, followed by an increase of amorphous silicates bands with increasing massloss rate, which start to grow on the Al-rich oxides (Lebzelter et al. 2006). A similar trend is seen in outflows of red supergiants (Verhoelst et al. 2009). A similar scenario might explain the lack of $\mathrm{Al} / \mathrm{Na} / \mathrm{Ca}$-rich dust species in the discs around the post-AGB stars. Since the photospheres of the central stars are strongly depleted in these elements (Maas et al. 2005; Hrivnak et al. 2008; Gielen et al. 2009b), we know these refractory elements must be present in the disc. Of course, since densities associated with these discs are much higher than for typical outflows, and dust might be subject to a different temperature gradient, which could result in a different condensation sequence to that observed in AGB stars.

\section{Conclusions}

We analysed the Spitzer infrared spectra of 33 Galactic and 24 LMC (candidate) post-AGB binaries surrounded by a dusty circumbinary disc. For nearly all Galactic sources, previous studies have already confirmed the binarity and post-AGB status. The LMC sources were taken from a list of probable postAGB disc candidates. Our main focus was to determine the dust composition of the discs, but also to look for possible differences between the Galactic and LMC sample. Our study shows that:

- The Spitzer spectra are all dominated by emission features of oxygen-rich dust species, namely amorphous and crystalline silicates of olivine and pyroxene stoichiometry.

- The observed silicate dust has a high crystallinity factor: most sources have crystalline mass fractions between $20-60 \%$.

- Most of the dust is stored in larger grains $(>2 \mu \mathrm{m})$. This results in an average grain size distribution of $n(a) \propto a^{-1.3_{0.2}^{0.1}}$ for grain sizes between 0.1 and $4 \mu \mathrm{m}$.

- We find no correlations between the dust, stellar, and/or orbital parameters, which makes it difficult to constrain the dust grain processes that are causing the observed dust properties such as the grain sizes and crystallinity.

- We find no differences between the dust parameters of the Galactic and LMC sources.

- Although the observed spectra are very similar to those of protoplanetary dics, we find evidence for a fundamental difference in the dust processing occurring in the two disc types, more specifically in the homogeneous dust compostion throughout the disc, the observed degree of crystallinity, the crystalline grain sizes and, the strong dominance of forsterite in the crystalline grain fraction.

\section{References}

Abia, C., \& Isern, J. 2000, ApJ, 536, 438

Becklin, E. E., Farihi, J., Jura, M., et al. 2005, ApJ, 632, L119

Bohren, C. F., Huffman, D. R., \& Kam, Z. 1983, Nature, 306, 625

Bouwman, J., Henning, T., Hillenbrand, L. A., et al. 2008, ApJ, 683, 479

Bujarrabal, V., Castro-Carrizo, A., Alcolea, J., \& Sánchez Contreras, C. 2001, A\&A, 377, 868

Bujarrabal, V., Van Winckel, H., Neri, R., et al. 2007, A\&A, 468, L45

Cami, J., Bernard-Salas, J., Peeters, E., \& Malek, S. E. 2010, Science, 329, 1180
Chesneau, O., Collioud, A., De Marco, O., et al. 2006, A\&A, 455, 1009

Chesneau, O., Lykou, F., Balick, B., et al. 2007, A\&A, 473, L29

Chihara, H., Koike, C., Tsuchiyama, A., Tachibana, S., \& Sakamoto, D. 2002,

A\&A, 391, 267

Chiu, P., Hoang, C., Dinh-V-Trung, Lim, J., et al. 2006, ApJ, 645, 605

De Ruyter, S., Van Winckel, H., Maas, T., et al. 2006, A\&A, 448, 641

Deroo, P. 2007, Ph.D. Thesis, KU Leuven

Deroo, P., Van Winckel, H., Min, M., et al. 2006, A\&A, 450, 181

Deroo, P., Van Winckel, H., Verhoelst, T., et al. 2007, A\&A, 467, 1093

Dong, R., Wang, Y., Lin, D. N. C., \& Liu, X. 2010, ApJ, 715, 1036

Dorschner, J., Begemann, B., Henning, T., Jaeger, C., \& Mutschke, H. 1995, A\&A, 300, 503

Dullemond, C. P., \& Dominik, C. 2004, A\&A, 417, 159

Fabian, D., Jäger, C., Henning, T., Dorschner, J., \& Mutschke, H. 2000, A\&A, 364,282

Gail, H. 2004, A\&A, 413, 571

Gielen, C., Van Winckel, H., Waters, L. B. F. M., Min, M., \& Dominik, C. 2007, A\&A, 475, 629

Gielen, C., Van Winckel, H., Min, M., Waters, L. B. F. M., \& Lloyd Evans, T. 2008, A\&A, 490, 725

Gielen, C., van Winckel, H., Matsuura, M., et al. 2009a, A\&A, 503, 843

Gielen, C., Van Winckel, H., Reyniers, M., et al. 2009b, A\&A, 508, 1391

Gielen, C., van Winckel, H., Min, M., et al. 2010, A\&A, 515, C2

Groenewegen, M. A. T., Wood, P. R., Sloan, G. C., et al. 2007, MNRAS, 376, 313

Henning, T., \& Mutschke, H. 1997, A\&A, 327, 743

Hines, et al. 2005, FEPS Data Explanatory Supplement, Version 3.0, Pasadena SSC

Houck, J. R., Roellig, T. L., van Cleve, J., et al. 2004, ApJS, 154, 18

Hrivnak, B. J., Van Winckel, H., Reyniers, M., et al. 2008, AJ, 136, 1557

Jaeger, C., Molster, F. J., Dorschner, J., et al. 1998, A\&A, 339, 904

Juhász, A., Bouwman, J., Henning, T., et al. 2010, ApJ, 721, 431

Jura, M. 2003, ApJ, 582, 1032

Jura, M., \& Kahane, C. 1999, ApJ, 521, 302

Kastner, J. H., Buchanan, C., Sahai, R., Forrest, W. J., \& Sargent, B. A. 2010, AJ, 139, 1993

Käufl, H.-U., Sterzik, M. F., Siebenmorgen, R., et al. 2003, in Instrument Design and Performance for Optical/Infrared Ground-based Telescopes, ed. M. Iye, \& A. F. M. Moorwood, SPIE Conf., 4841, 117

Kemper, F., Woods, P. M., Antoniou, V., et al. 2010, PASP, 122, 683

Kurucz, R. L. 1979, ApJS, 40, 1

Lahuis, et al. 2006, c2d Spectroscopy Explanatory Supplement, Cores to Disks, Legacy Team (Pasadena: Spitzer, Science Center)

Lebzelter, T., Posch, T., Hinkle, K., Wood, P. R., \& Bouwman, J. 2006, ApJ, 653, L145

Lloyd Evans, T. 1990, MNRAS, 243, 336

Lloyd Evans, T. 1997, Ap\&SS, 251, 239

Lloyd Evans, T. 1999, in Asymptotic Giant Branch Stars, ed. T. Le Bertre, A. Lebre, \& C. Waelkens, IAU Symp., 191, 453

Lykou, F., Chesneau, O., Zijlstra, A. A., et al. 2011, A\&A, 527, A105

Maas, T., Van Winckel, H., \& Lloyd Evans, T. 2003, in ASP Conf. Ser., 303, ed. R. L. M. Corradi, J. Mikolajewska, \& T. J. Mahoney, 143

Maas, T., Van Winckel, H., \& Lloyd Evans, T. 2005, A\&A, 429, 297

Mathis, J. S., Rumpl, W., \& Nordsieck, K. H. 1977, ApJ, 217, 425

Meeus, G., Waters, L. B. F. M., Bouwman, J., et al. 2001, A\&A, 365, 476

Meixner, M., Gordon, K. D., Indebetouw, R., et al. 2006, AJ, 132, 2268

Melis, C., Gielen, C., Chen, C. H., et al. 2010, ApJ, 724, 470

Menzies, J. W., \& Whitelock, P. A. 1988, MNRAS, 233, 697

Min, M., Hovenier, J. W., \& de Koter, A. 2005, A\&A, 432, 909

Min, M., Dominik, C., Hovenier, J. W., de Koter, A., \& Waters, L. B. F. M. 2006 , A\&A, 445, 1005

Min, M., Hovenier, J. W., Waters, L. B. F. M., \& de Koter, A. 2008, A\&A, 489, 135

Molster, F. J., Waters, L. B. F. M., \& Tielens, A. G. G. M. 2002a, A\&A, 382 222

Molster, F. J., Waters, L. B. F. M., Tielens, A. G. G. M., Koike, C., \& Chihara, H. 2002b, A\&A, 382, 241

Morris, M. 1990, in From Miras to Planetary Nebulae: Which Path for Stellar Evolution?, ed. M. O. Mennessier, \& A. Omont, 520

Petaev, M. I., \& Wood, J. A. 2005, in Chondrites and the Protoplanetary Disk, ed. A. N. Krot, E. R. D. Scott, \& B. Reipurth, ASP Conf. Ser., 341, 373

Reimann, H.-G., Linz, H., Wagner, R., et al. 2000, in Optical and IR Telescope Instrumentation and Detectors, ed. M. Iye, \& A. F. Moorwood, SPIE Conf., 4008, 1132

Reyniers, M., \& Van Winckel, H. 2007, A\&A, 463, L1

Sargent, B. A., Forrest, W. J., Tayrien, C., et al. 2009, ApJ, 690, 1193

Savage, B. D., \& Mathis, J. S. 1979, ARA\&A, 17, 73

Servoin, J. L., \& Pirou, B. 1973, Phys. Stat. Sol. B, 55, 677 
Shkuratov, Y. G. \& Grynko, Y. S. 2005, Icarus, 173, 16

Srinivasan, S., Sargent, B. A., \& Meixner, M. 2011, A\&A, 532, A54

Stancliffe, R. J., Izzard, R. G., \& Tout, C. A. 2005, MNRAS, 356, L1

Steenman, H., \& Thé, P. S. 1989, Ap\&SS, 159, 189

Steenman, H., \& Thé, P. S. 1991, Ap\&SS, 184, 9

Tielens, A. G. G. M. 1990, From Miras to Planetary Nebulae, ed. M. O. Mennessier, \& A. Omont (Éditions Frontières), 186

Tielens, A. G. G. M., Waters, L. B. F. M., Molster, F. J., \& Justtanont, K. 1998, Ap\&SS, 255, 415

van Aarle, E., Van Winckel, H., Lloyd Evans, T., et al. 2011, A\&A, 530, A90

van Boekel, R., Waters, L. B. F. M., Dominik, C., et al. 2003, A\&A, 400, L2

van Boekel, R., Min, M., Waters, L. B. F. M., et al. 2005, A\&A, 437, 189
Van Winckel, H., Lloyd Evans, T., Briquet, M., et al. 2009, A\&A, 505, 1221

Verhoelst, T., van Aarle, E., \& Acke, B. 2007, A\&A, 470, L21

Verhoelst, T., van der Zypen, N., Hony, S., et al. 2009, A\&A, 498, 127

Waters, L. B. F. M., Trams, N. R., \& Waelkens, C. 1992, A\&A, 262, L37

Werner, M. W., Roellig, T. L., Low, F. J., et al. 2004, ApJS, 154, 1

Wooden, D. H., Harker, D. E., \& Brearley, A. J. 2005, in Chondrites and the Protoplanetary Disk, ed. A. N. Krot, E. R. D. Scott, \& B. Reipurth, ASP Conf. Ser., 341, 774

Woods, P. M., Oliveira, J. M., Kemper, F., et al. 2011, MNRAS, 411, 1597

Yamamura, I., Dominik, C., de Jong, T., Waters, L. B. F. M., \& Molster, F. J. 2000, A\&A, 363, 629

Zubko, V., Dwek, E., \& Arendt, R. G. 2004, ApJS, 152, 211

Pages 15 to 26 are available in the electronic edition of the journal at http: //www . aanda. org 
C. Gielen et al.: Silicate features in Galactic and extragalactic post-AGB disks

\section{Appendix A: Figures and Tables}
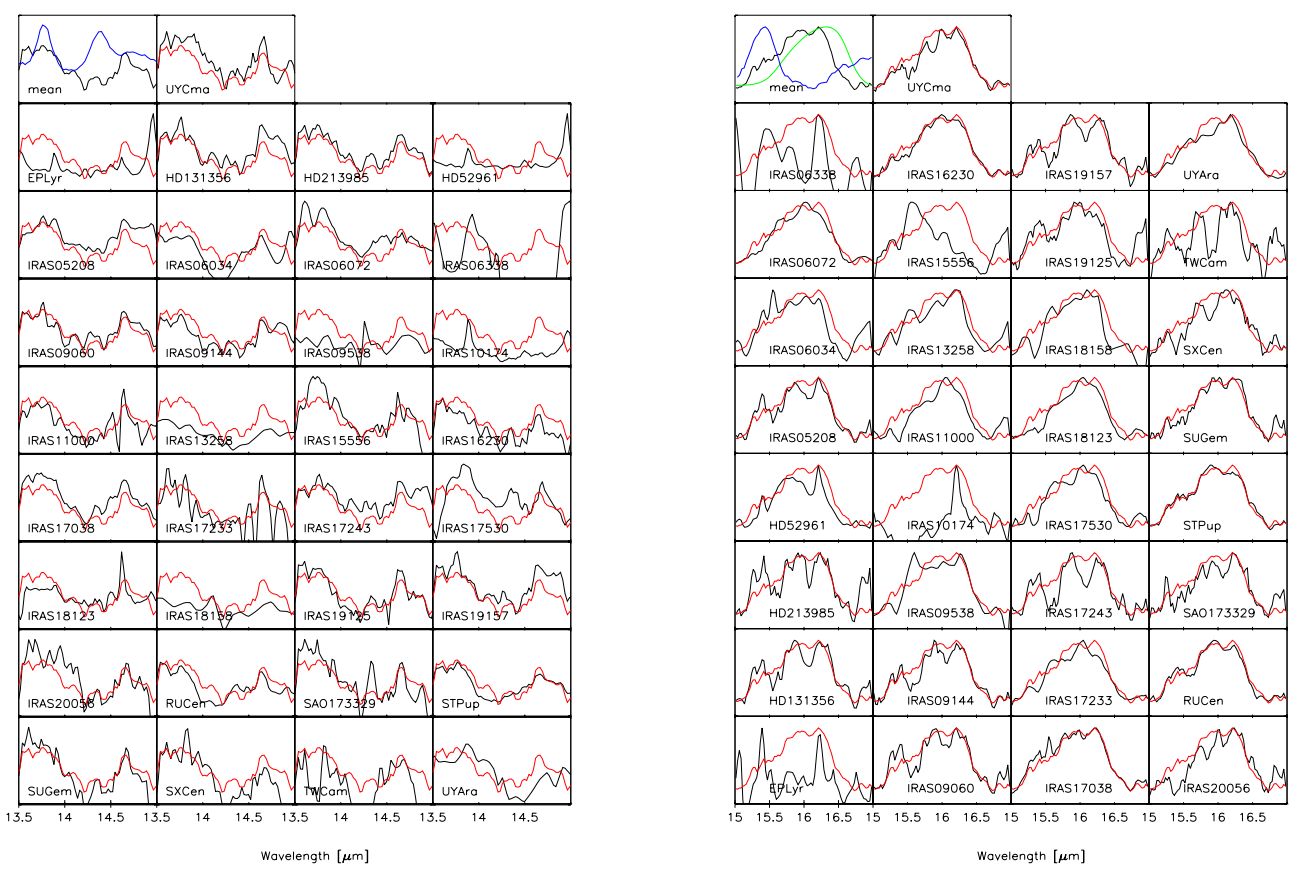

Fig. A.1. Left: the $14 \mu \mathrm{m}$ complex for the Galactic sources, continuum subtracted and normalised. Overplotted in red the mean spectrum. The mass absorption coefficients of forsterite and enstatite are plotted in green and blue. The high noise level on the LMC sources did not allow for a mean spectrum determination. Right: same as on the left, but for the $16 \mu \mathrm{m}$ complex.
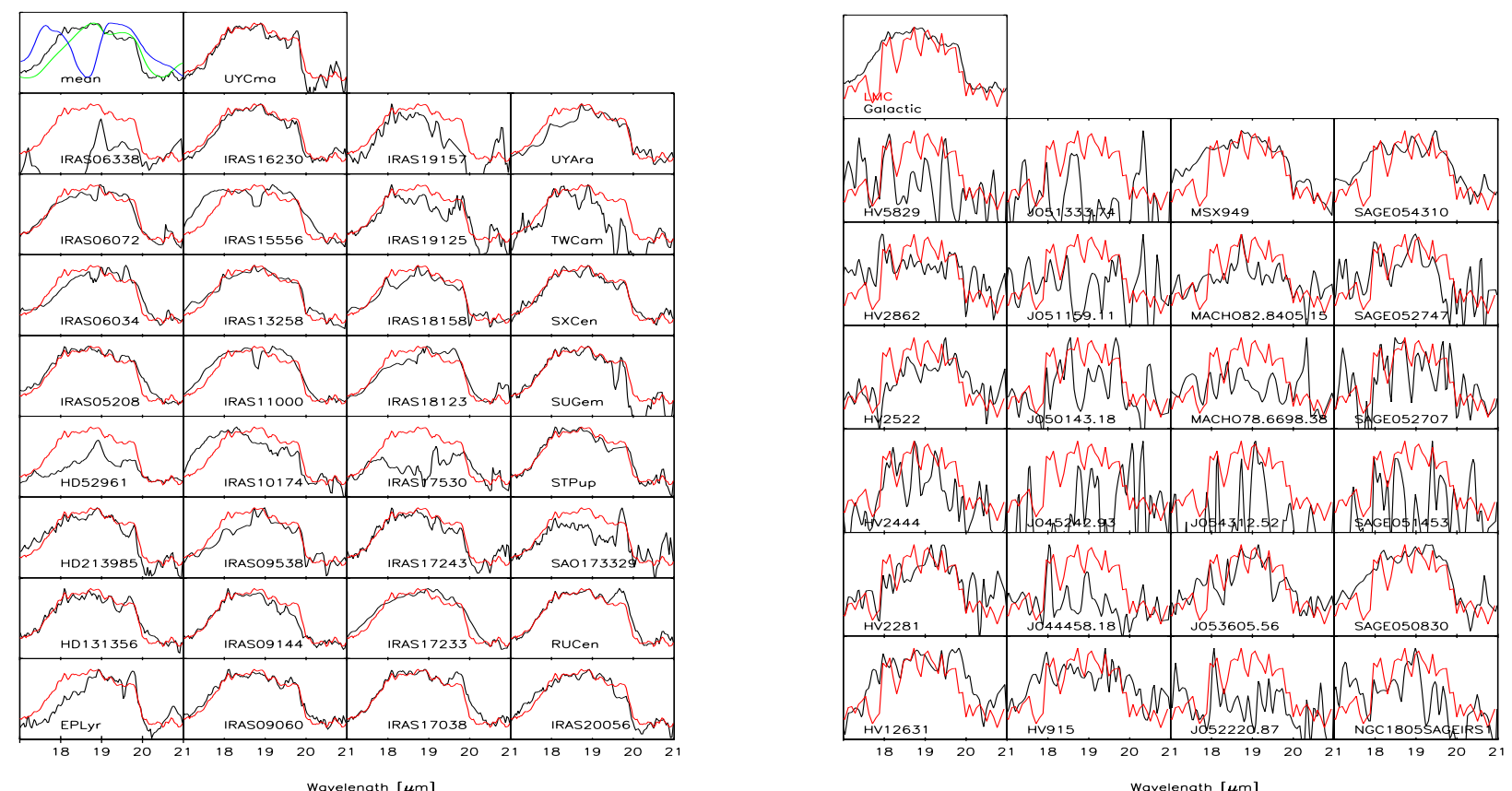

Fig. A.2. Left: the $19 \mu \mathrm{m}$ complex for the Galactic sources, continuum subtracted and normalised. Overplotted in red the mean spectrum. The mass absorption coefficients of forsterite and enstatite are plotted in green and blue. Right: same as on the left, but for the LMC sources. The top panel shows the comparison between the calculated mean for the LMC and Galactic sources. 


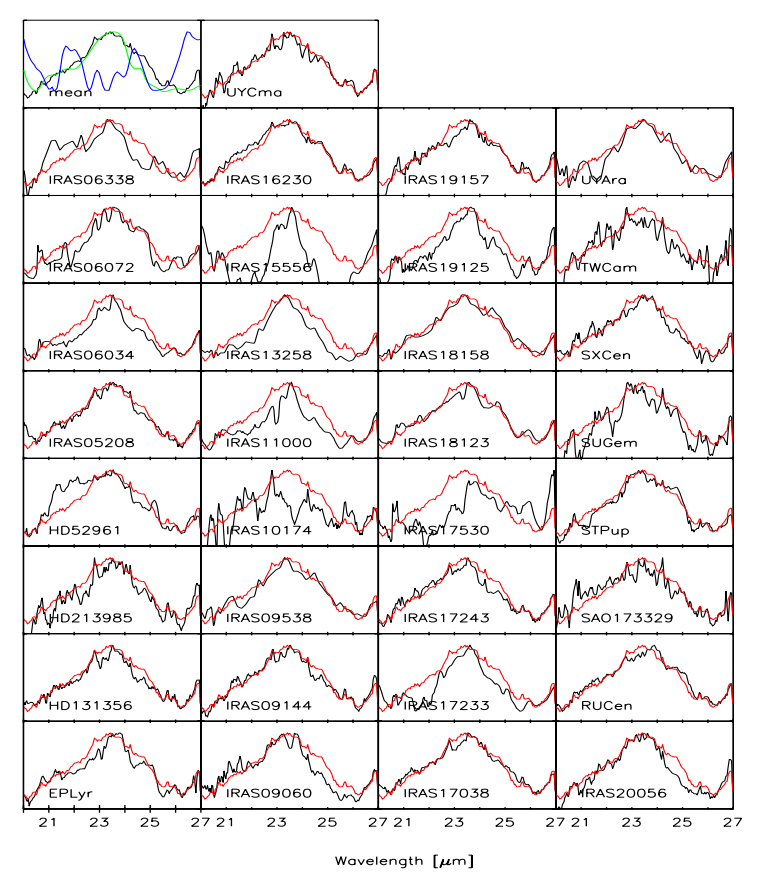

Fig. A.3. Same as Fig. A.2, but for the $23 \mu \mathrm{m}$ complex.

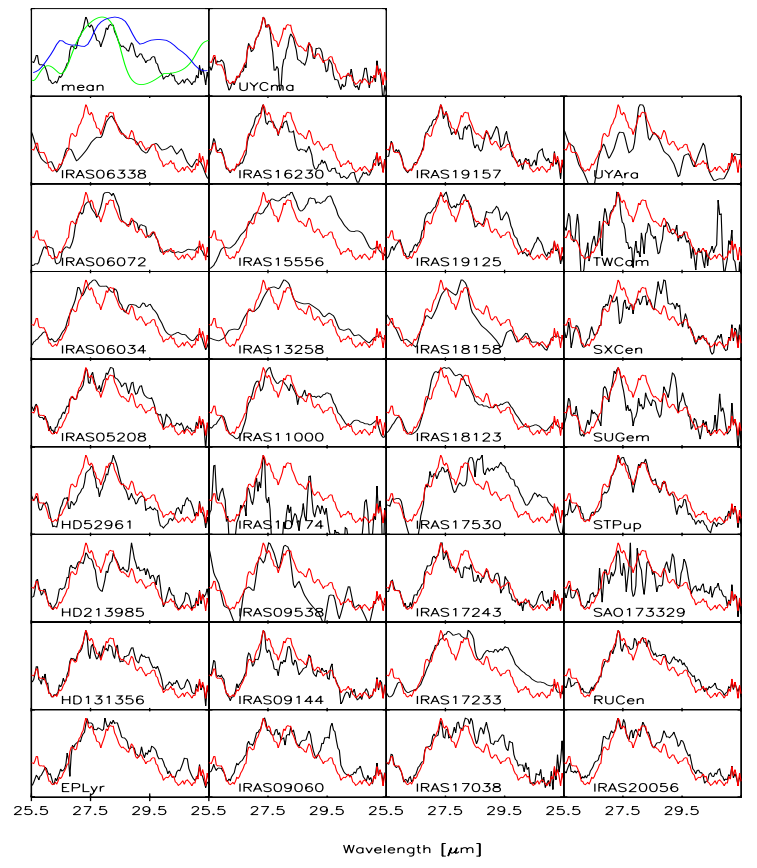

Fig. A.4. Same as Fig. A.2, but for the $27 \mu \mathrm{m}$ complex.
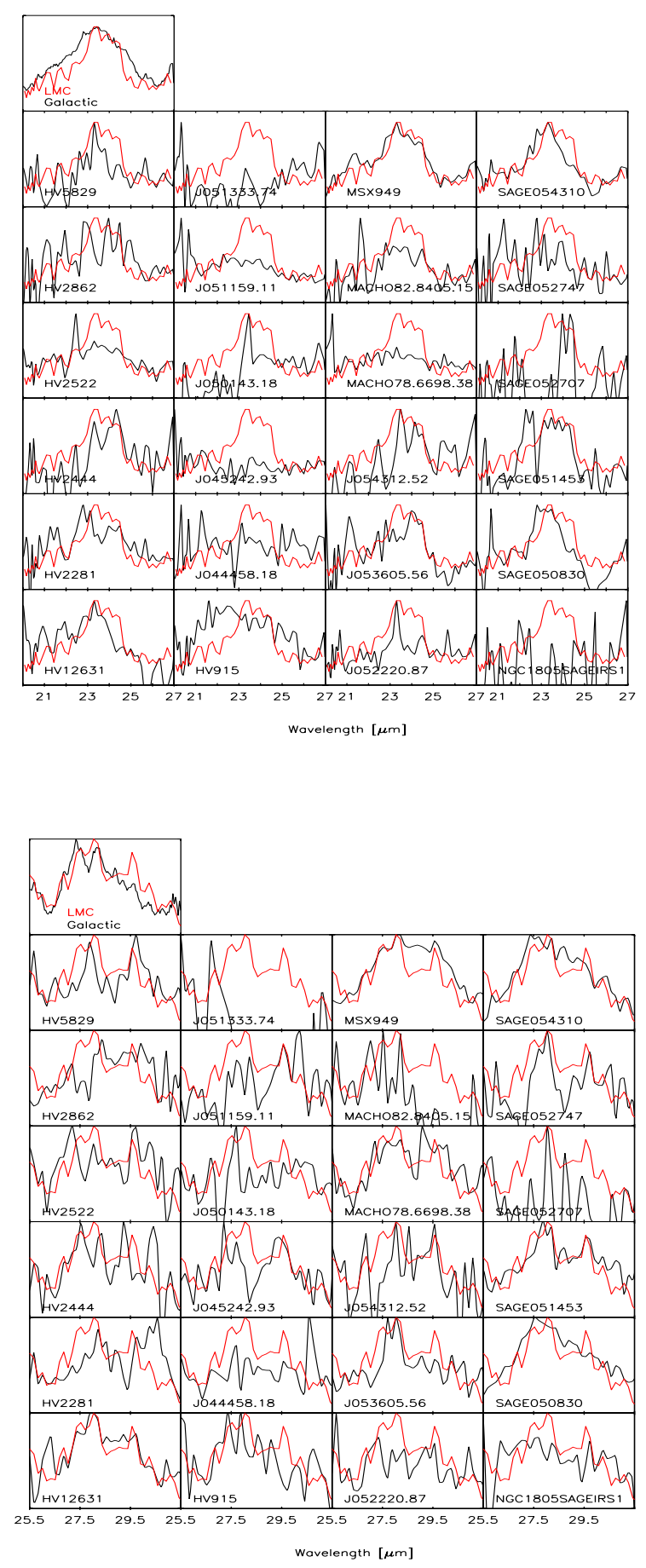

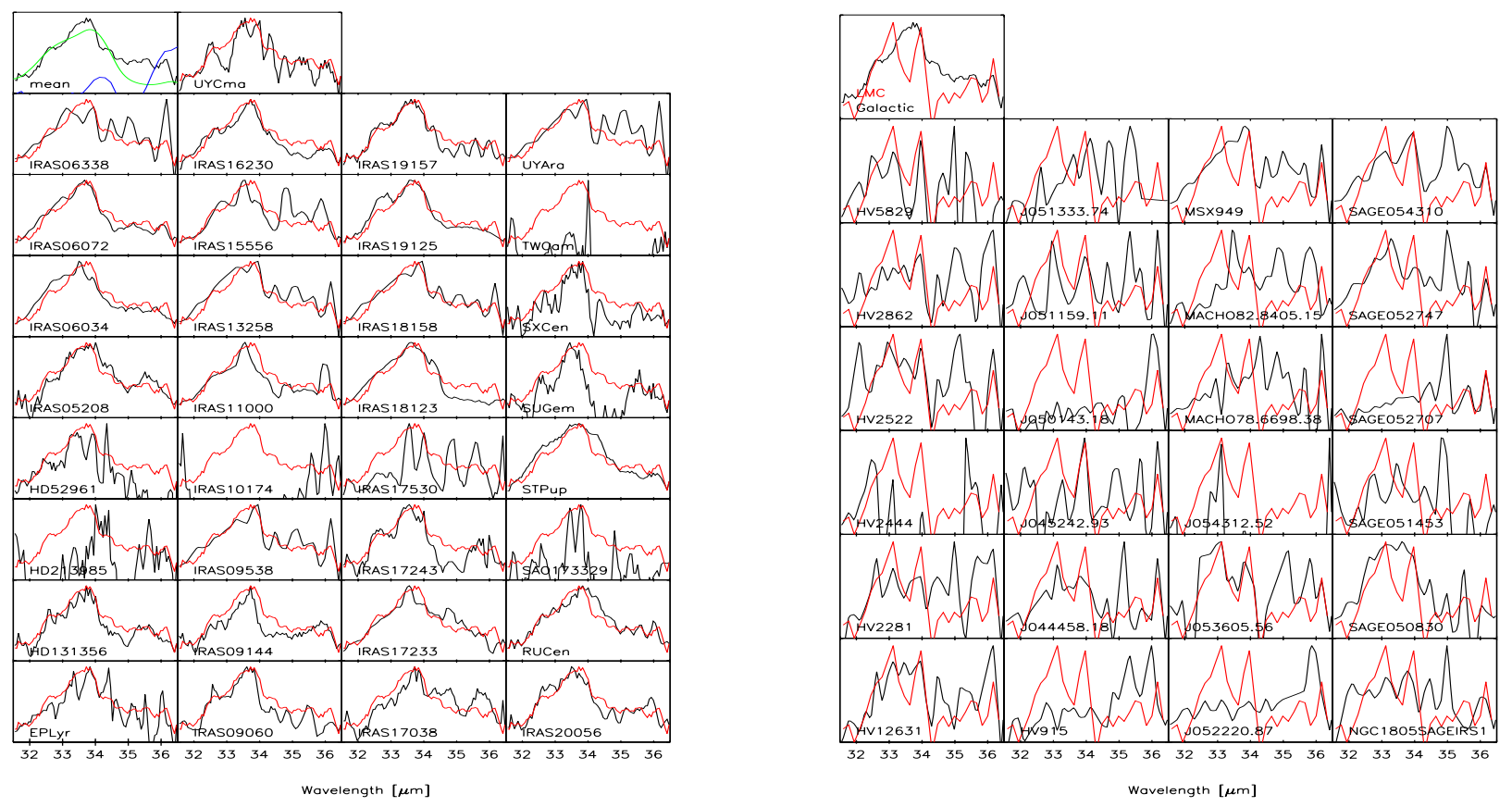

Fig. A.5. Same as Fig. A.2, but for the $33 \mu \mathrm{m}$ complex.
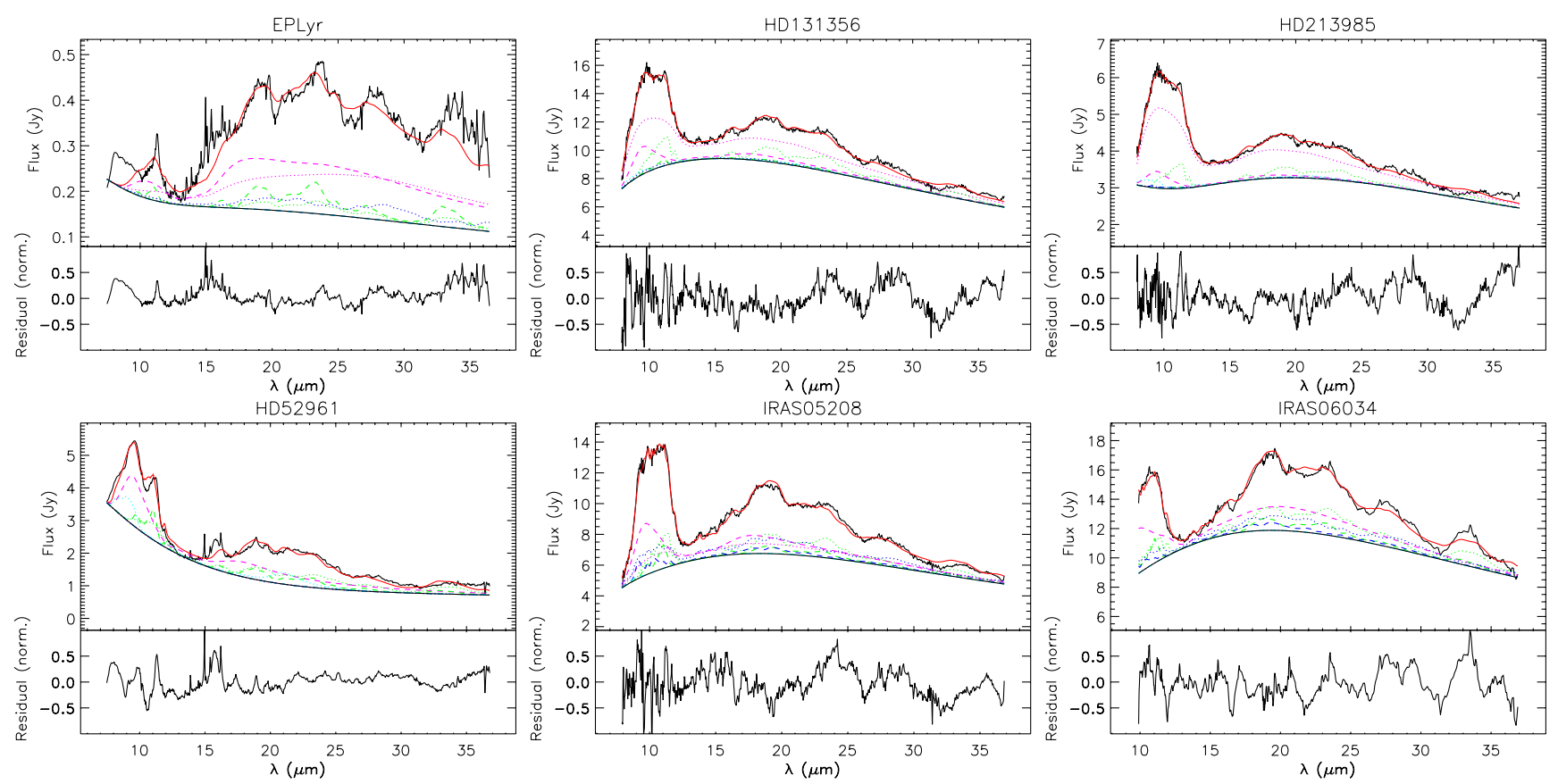

Fig. A.6. Best model fits for our Galactic sample stars, showing the contribution of the different dust species. Top: the observed spectrum (black curve) is plotted together with the best model fit (red curve) and the continuum (black solid line). Forsterite is plotted in green, enstatite in blue, silica in cyan and amorphous olivine and pyroxene in magenta. Small grains $(0.1 \mu \mathrm{m})$ are plotted as dashed lines and larger grains $(2$ and $4 \mu \mathrm{m})$ as dotted lines. Bottom: the normalised residuals after subtraction of our best model of the observed spectra. 

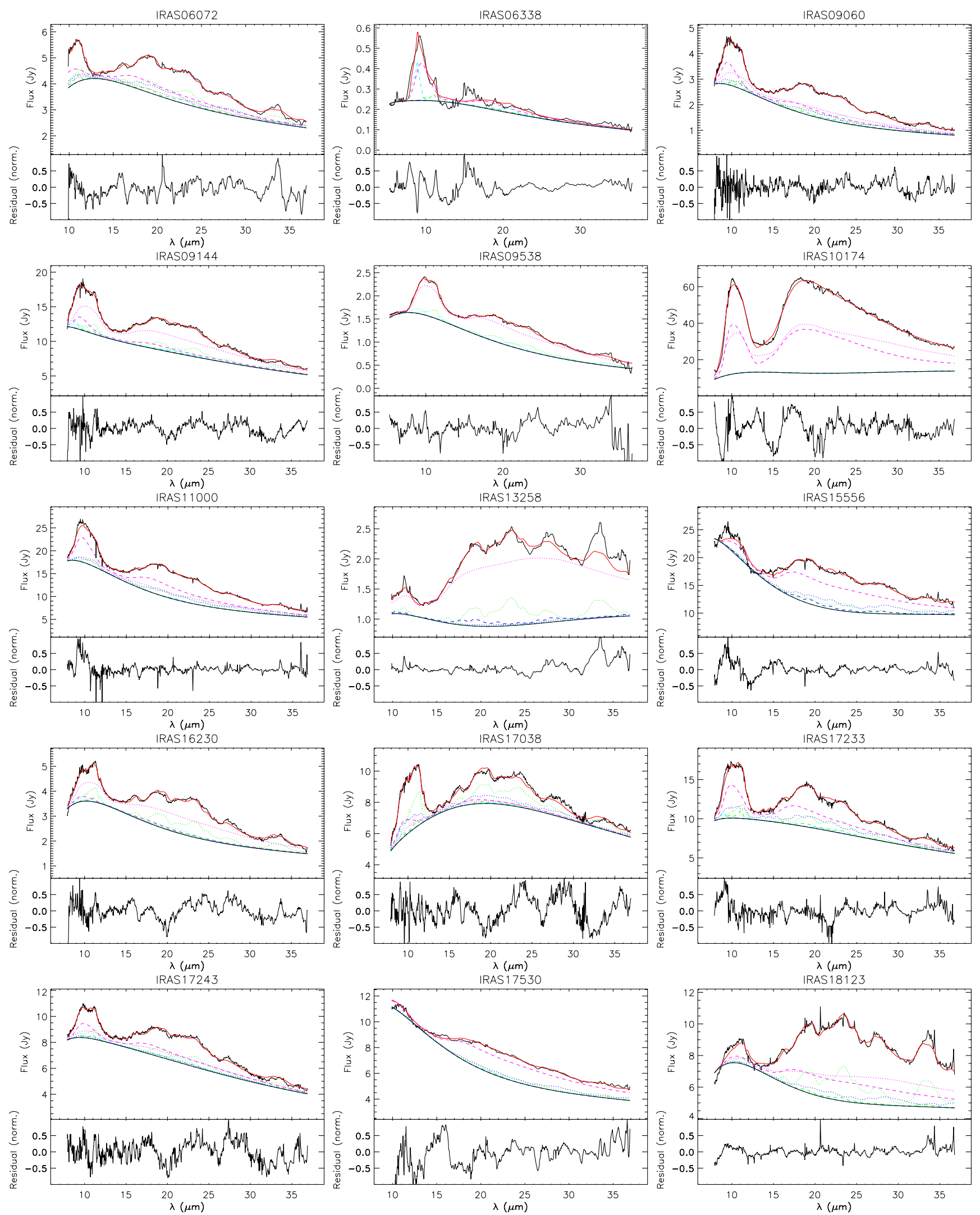

Fig. A.6. continued. 
C. Gielen et al.: Silicate features in Galactic and extragalactic post-AGB disks
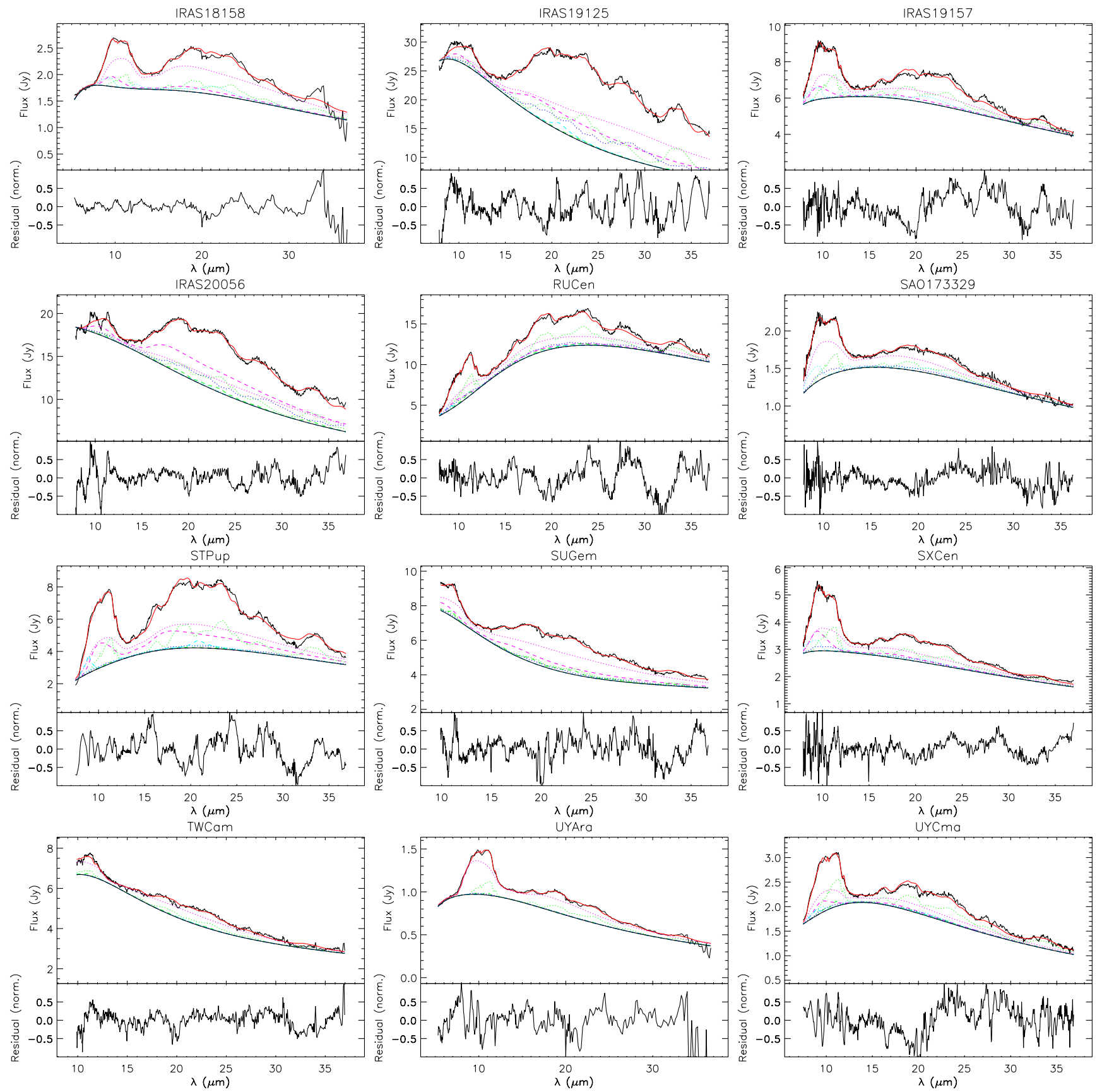

Fig. A.6. continued. 

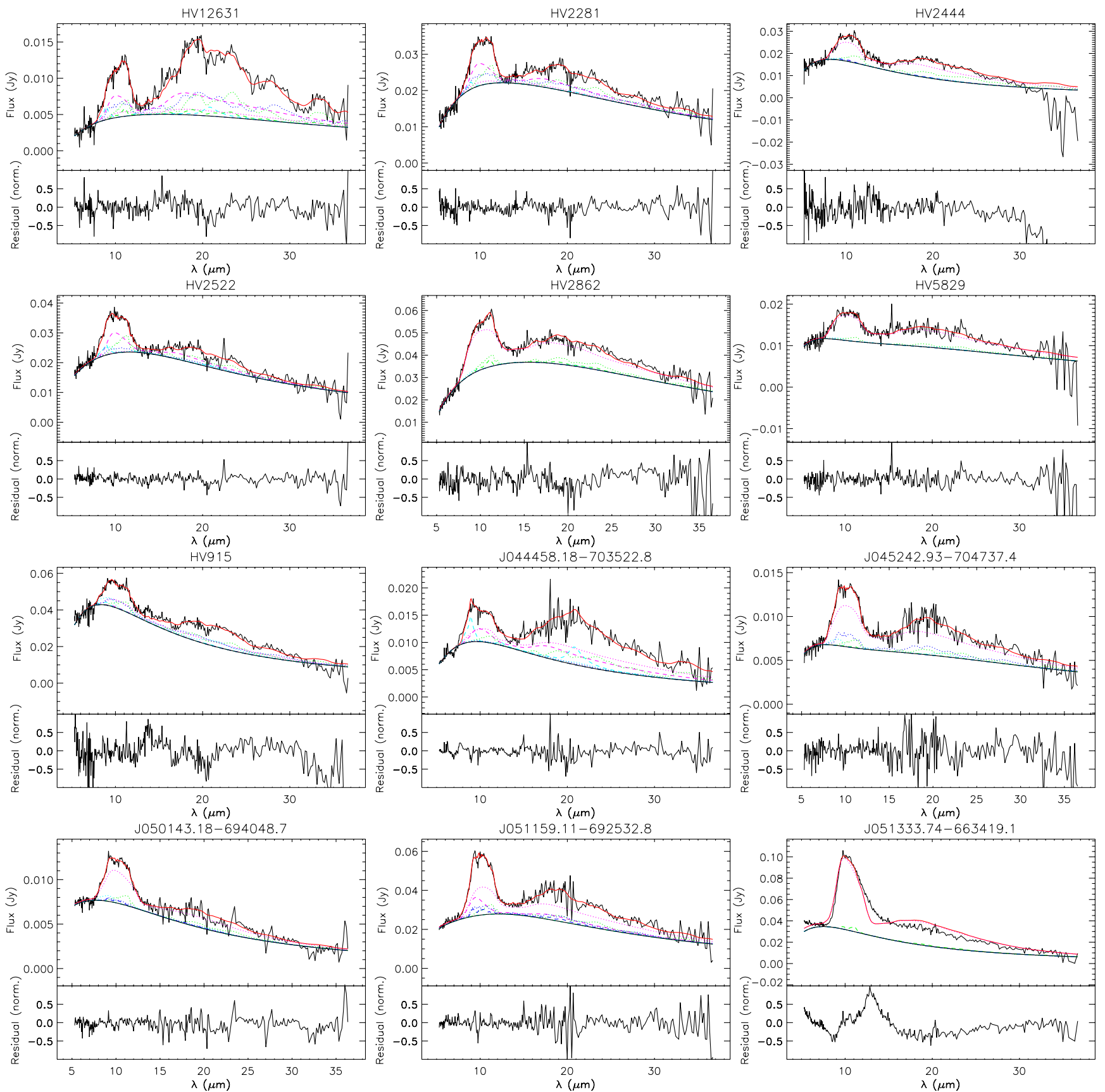

Fig. A.7. Best model fits for our LMC sample stars, showing the contribution of the different dust species. Top: the observed spectrum (black curve) is plotted together with the best model fit (red curve) and the continuum (black solid line). Forsterite is plotted in green, enstatite in blue, silica in cyan and amorphous olivine and pyroxene in magenta. Small grains $(0.1 \mu \mathrm{m})$ are plotted as dashed lines and larger grains $(2$ and $4 \mu \mathrm{m})$ as dotted lines. Bottom: the normalised residuals after subtraction of our best model of the observed spectra. 
C. Gielen et al.: Silicate features in Galactic and extragalactic post-AGB disks
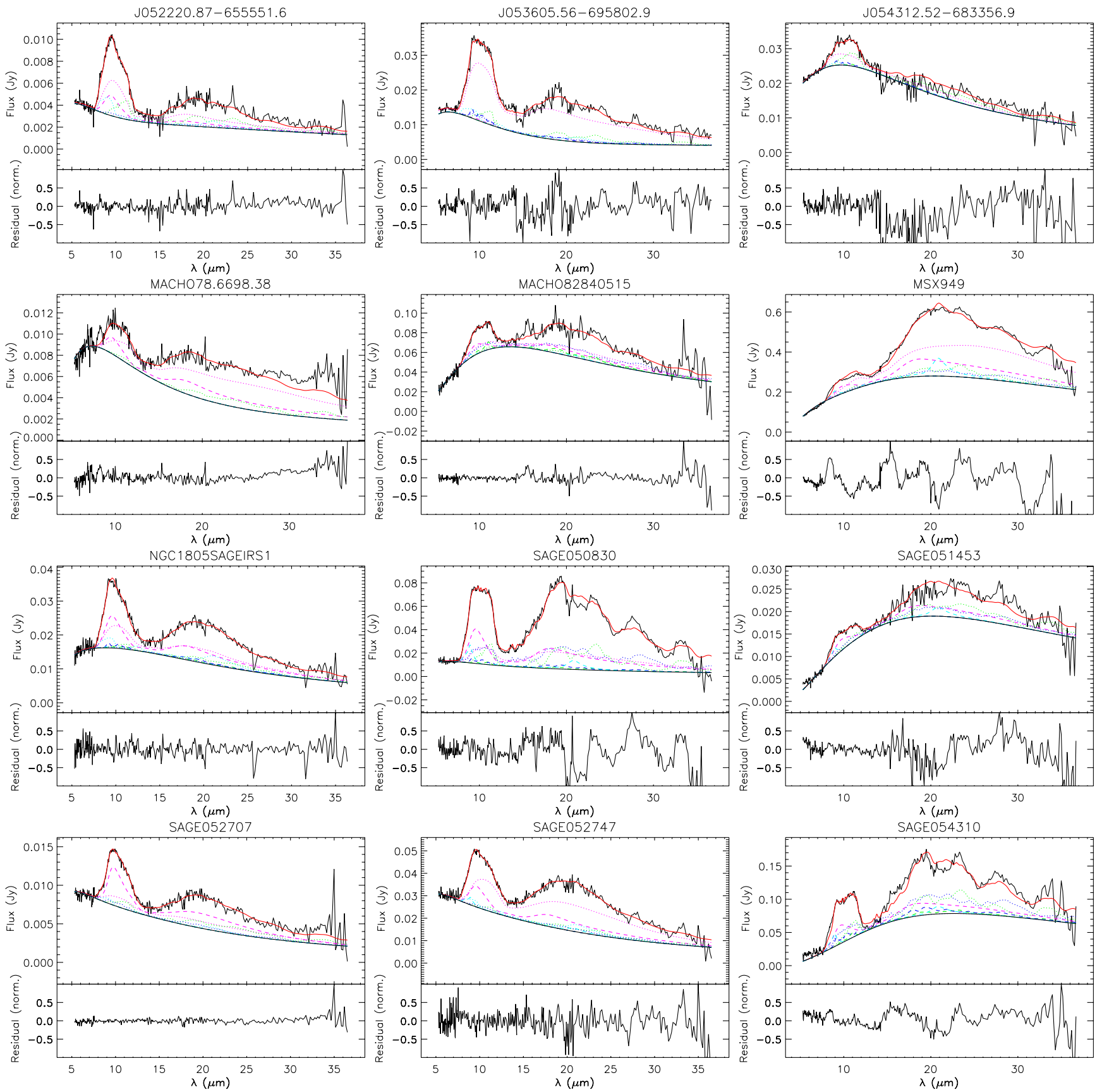

Fig. A.7. continued. 

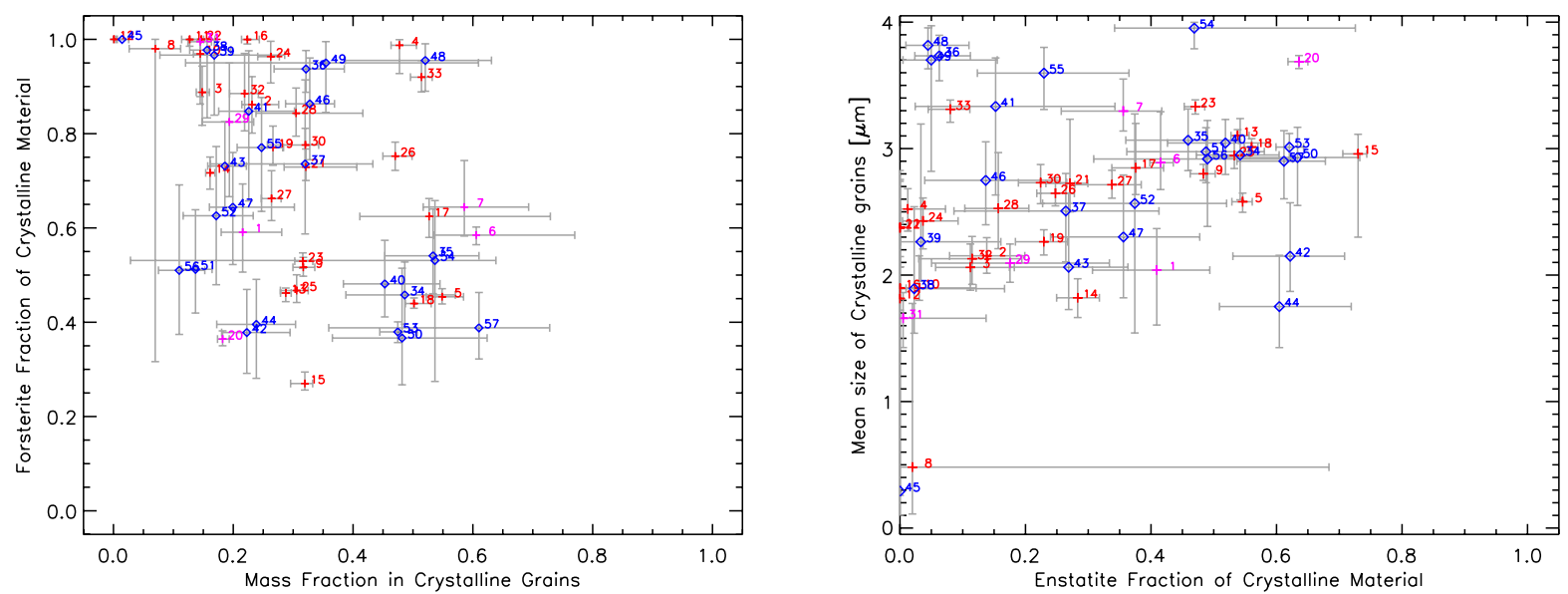

Fig. A.8. Left: the mass fraction in crystalline grains versus the forsterite fraction in the crystalline material. Right: the mean size of crystalline grains versus the enstatite fraction in the crystalline material. Galactic sources are given in red plus signs and LMC sources in blue diamonds. The magenta symbols depict Galactic sources for which the infrared spectra only start from $9.9 \mu \mathrm{m}$. The numbers correspond to numbers given in Tables 1 and 2 .
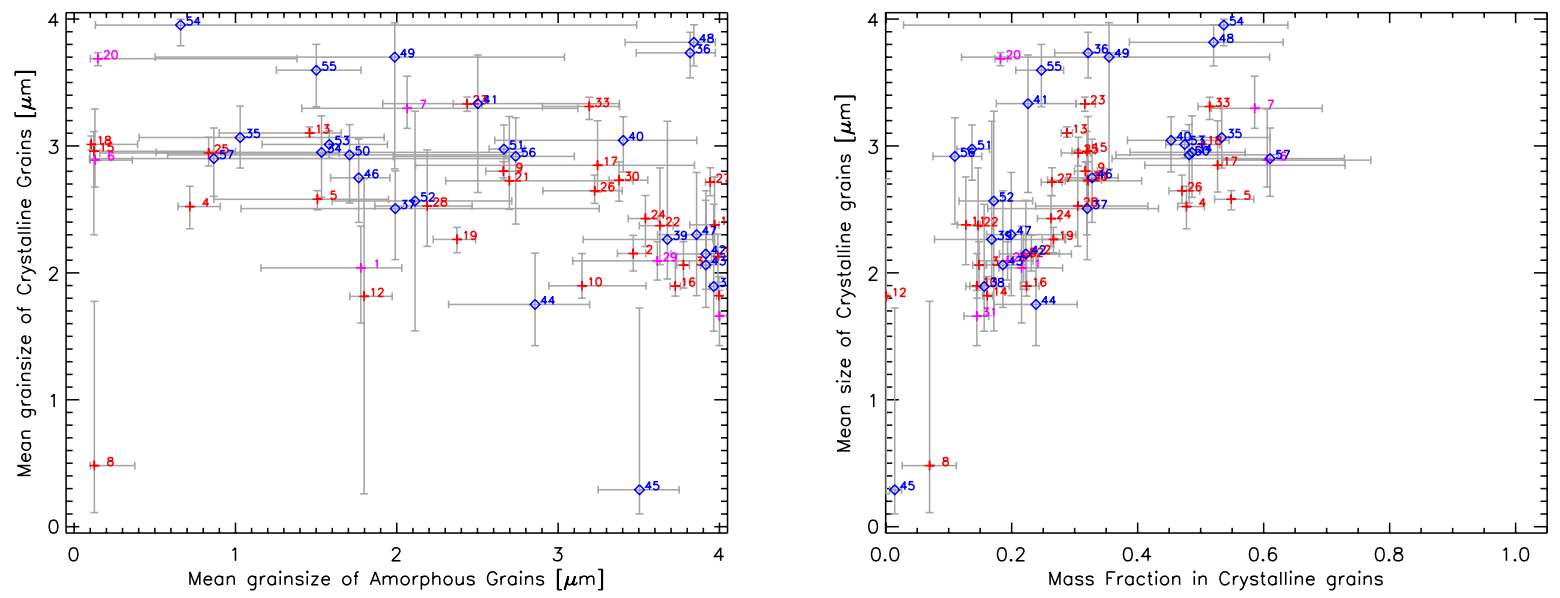

Fig. A.9. Left: the mean size of crystalline grains versus the mean size of amorphous grains. Right: the mean size of the crystalline grains versus crystallinity fraction. Galactic sources are given in red plus signs and LMC sources in blue diamonds. The magenta symbols depict Galactic sources for which the infrared spectra only start from $9.9 \mu \mathrm{m}$. The numbers correspond to numbers given in Tables 1 and 2 . 
C. Gielen et al.: Silicate features in Galactic and extragalactic post-AGB disks

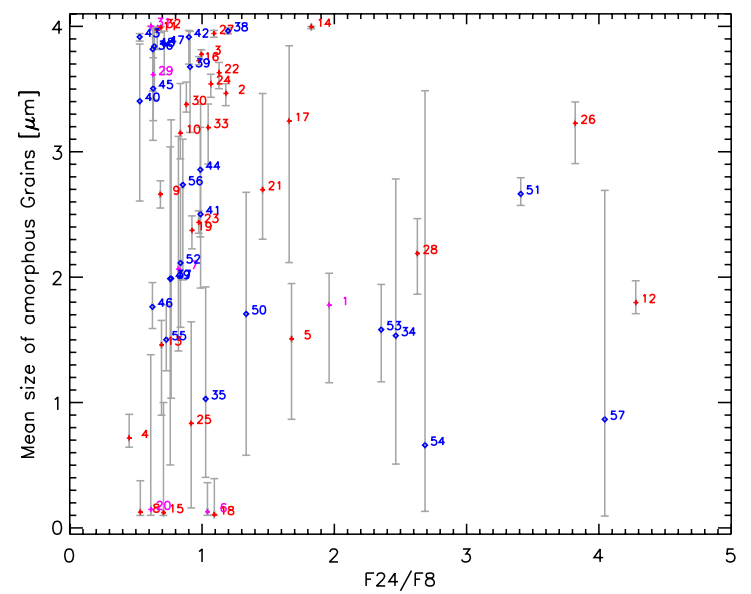

Fig. A.10. The mean size of the amorphous grains versus the disc flaring, determined by the $F_{24} / F_{8}$ flux ratio. Galactic sources are given in red plus signs and LMC sources in blue diamonds. The magenta symbols depict Galactic sources for which the infrared spectra only start from $9.9 \mu \mathrm{m}$. The numbers correspond to numbers given in Tables 1 and 2. 
Table A.1. Best fit parameters deduced from our full spectral fitting.

\begin{tabular}{|c|c|c|c|c|c|c|c|}
\hline $\mathrm{N}^{\circ}$ & Name & $\begin{array}{l}T_{\text {dust1 }} \\
(\mathrm{K})\end{array}$ & $\begin{array}{l}T_{\text {dust2 }} \\
(\mathrm{K})\end{array}$ & $\begin{array}{c}\text { Fraction } \\
T_{\text {dust } 1}-T_{\text {dust } 2}\end{array}$ & $\begin{array}{c}T_{\text {cont1 }} \\
(\mathrm{K})\end{array}$ & $\begin{array}{l}T_{\text {cont2 }} \\
(\mathrm{K})\end{array}$ & $\begin{array}{c}\text { Fraction } \\
T_{\text {cont1 } 1}-T_{\text {cont2 }}\end{array}$ \\
\hline 1 & EPLyr & 100.0 & 200.0 & $0.90_{0.00}^{0.00}-0.10_{0.00}^{0.00}$ & 200.0. & 996.4. & $0.98_{0.00}^{0.01}-0.02_{0.01}^{0.00}$ \\
\hline 2 & HD 131356 & 200. & 1000. & $0.90_{0.10}^{8.00}-0.10^{8.100}$ & 200. & 500. & $0.90_{0.00}^{8.80}-0.10^{0.80}$ \\
\hline 3 & HD 213985 & 100.0. & 1000.0. & $0.90_{0.00}^{0.00}-0.10_{0.00}^{0.00}$ & 200.0 & 800.0 & $0.98_{0.00}^{0.00}-0.02_{0.00}^{0.00}$ \\
\hline 4 & HD 52961 & 200. & 704..$_{4}^{111}$ & $0.90_{0.00}^{0.00}-0.10_{0.00}^{0.00}$ & 100.0 & $1000_{0}^{0 .}$ & $0.99_{0.00}^{8.80}-0.01_{0.00}^{8.80}$ \\
\hline 5 & IRAS 05208 & 426..$_{109}$ & 771.204. & $0.70_{0.30}^{0.20}-0.30_{0.20}^{0.30}$ & 200.0 & 500.0. & $0.93_{0.01}^{0.00}-0.07_{0.00}^{0.01}$ \\
\hline 6 & IRAS 06034 & 200. & 530.71 & $0.90^{0.00}-0.10^{0.00}$ & 200. & 500.0 & $0.94^{0.00}-0.06^{0.00}$ \\
\hline 7 & IRAS 06072 & 200.0 & 680.296. & $0.90^{0.00}-0.10_{0.00}^{0.00}$ & 178.124. & 564.263. & $0.94_{0.02}^{0.04}-0.06_{0.04}^{0.02}$ \\
\hline 8 & IRAS 06338 & 179.459 & 1000.0 & $0.60_{0.50}^{0.30}-0.40_{0.30}^{8.00}$ & 277.23 . & $\begin{array}{l}859.158 . \\
8157\end{array}$ & $0.94_{0.01}^{8.81}-0.06_{0.01}^{0.84}$ \\
\hline 9 & IRAS 09060 & 200. & $600.0^{0}$ & $0.90^{0.00}-0.10^{0.00}$ & 100.0. & 600. & $0.95^{8.00}-0.05^{0.81}$ \\
\hline 10 & IRAS 09144 & 209.96 & 627.290. & $0.90_{0.00}^{8000}-0.10^{8000}$ & 200. & 700. & $0.93^{8.00}-0.07^{0.001}$ \\
\hline 11 & IRAS 09538 & 274.319. & 699.10: & $0.80_{0.40}^{0.10}-0.20_{0.40}^{0.40}$ & 376.24 & 976.24 & $0.91_{0.01}^{0.01}-0.09^{0.01}$ \\
\hline 12 & IRAS 10174 & 100.0 & 300.0 & $0.90_{0.00}^{0.00}-0.10_{0.00}^{0.00}$ & 100.0. & 423.79 & $0.98^{0.01}-0.02^{0.00}$ \\
\hline 13 & IRAS 11000 & 177.23 & 426.75 & $0.80_{0}^{0.10}-0.20_{010}^{0.00}$ & 100. & $603.3^{119}$ & $0.96^{0.02}-0.04^{0.00}$ \\
\hline 14 & IRAS 13258 & 100. & 200. & $0.80_{0.00}^{0.00}-0.20^{0.00}$ & 100.0 & 500. & $0.99^{0.00}-0.01^{0.00}$ \\
\hline 15 & IRAS 15556 & 100.0 & 200.0 & $0.20_{010}^{0.00}-0.80_{05}^{0.10}$ & 100.0 & 689. 11. & $0.99^{0.00}-0.01^{0.01}$ \\
\hline 16 & IRAS 16230 & 200.0 & 500.0 & $0.90_{0.00}^{0.10}-0.10_{0.00}^{0.50}$ & 100.0 & 500.03 & $0.95_{0.01}^{0.00}-0.05_{0.00}^{0.01}$ \\
\hline 17 & IRAS 17038 & 218.135. & 952.48 & $0.90_{0.10}^{0.00}-0.10_{0.00}^{0.00}$ & $200_{0}^{0 .}$ & 513. ${ }_{13}^{91 .}$ & $0.95_{0.01}^{0.01}-0.05_{0.01}^{0.00}$ \\
\hline 18 & IRAS 17233 & 320.82 & 563. 134. & $0.80_{010}^{0.10}-0.20_{0}^{0.10}$ & 200.0. & 600.0 & $0.92^{0.00}-0.08^{0.000}$ \\
\hline 19 & IRAS 17243 & 200.0 & 500.0 & $0.90_{0.00}^{0.00}-0.10^{0.000}$ & $2000^{0}$ & $600^{0}$ & $0.90_{0.00}^{8: 00}-0.10_{0.00}^{8.80}$ \\
\hline 20 & IRAS 17530 & 100. & 200. & $0.90_{0.10}^{0.00}-0.10_{000}^{0.010}$ & 100.0 & 600.0 & $0.97^{0.00}-0.03^{0.800}$ \\
\hline 21 & IRAS 18123 & 136.65 & 240.79 & $0.80_{0.10}^{0.10}-0.20_{0}^{0.10}$ & $100^{0} 0^{\circ}$ & 464.36. & $0.98^{0.00}-0.02^{0.00}$ \\
\hline 22 & IRAS 18158 & 201.106. & 414.201. & $0.60_{030}^{0.30}-0.40_{030}^{.028}$ & 200.0 & 700.0. & $0.96^{0.00}-0.04^{8.00}$ \\
\hline 23 & IRAS 19125 & 100.06 & 200. & $0.90_{0.00}^{0.00}-0.10_{0.00}^{0.00}$ & 500.0 & 900.0 & $0.91_{0.01}^{0: 00}-0.09^{0.01}$ \\
\hline 24 & IRAS 19157 & 200.0. & 799. 1. & $0.90_{0}^{0.00}-0.10_{0}^{0.000}$ & 200.0. & 601.0. & $0.94^{0.00}-0.06^{0.00}$ \\
\hline 25 & IRAS 20056 & 100.0 & 200.0 & $0.90^{0.00}-0.10^{0.10}$ & 304. & 850.66. & $0.91^{0.01}-0.09^{0.01}$ \\
\hline 26 & RUCen & $277.23:$ & $576^{24}$ & $0.90^{0.00}-0.10^{0.00}$ & $200^{\circ}$ & 596.4 & $0.99^{0.00}-0.01^{0.81}$ \\
\hline 27 & SAO 173329 & $101^{105}$ & 998. & $0.90_{0.00}^{0.00}-0.10^{0.000}$ & 200.0. & 501.11. & $0.90_{0.00}^{0.00}-0.10_{0.00}^{0.00}$ \\
\hline 28 & STPup & 203.19. & 487.25. & $0.80_{010}^{0.10}-0.20_{0}^{0.10}$ & 200.0. & 472.28. & $0.94_{0.04}^{0.01}-0.06_{0.01}^{0.00}$ \\
\hline 29 & SUGem & 213.358. & 506.176. & $0.80_{010}^{0.10}-0.20_{0}^{0: 10}$ & 158.42. & 776.130. & $0.97^{0.01}-0.03^{0.81}$ \\
\hline 30 & SXCen & 171.494. & 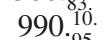 & $0.80_{0.10}^{0.10}-0.20_{0}^{0.00}$ & 200.0 & 617.86. & $0.93^{8.03}-0.07^{0.81}$ \\
\hline 31 & TWCam & 206.98. & 400.0. & $0.70_{0}^{0.10}-0.30_{0}^{0: 18}$ & 100.0 & 500. & $0.95^{0.00}-0.05^{0.000}$ \\
\hline 32 & UYAra & 219.37. & 869.53 & $0.70_{0.30}^{0.10}-0.30_{020}^{0.10}$ & 300.0. & 800.0. & $0.91^{0.00}-0.09^{0.000}$ \\
\hline 33 & UYCma & 200.0 & 1000.0 & $0.90_{0.00}^{0.00}-0.10_{0.00}^{0.00}$ & 300.0 & 900.0 & $0.97_{0.00}^{0.00}-0.03_{0.00}^{0.00}$ \\
\hline 34 & HV 12631 & 189.16. & $382.40^{\circ}$ & $0.80_{0.50}^{0.10}-0.20_{0.10}^{0.50}$ & 232.69 & 605.147. & $0.93_{0.03}^{0.02}-0.07_{0.02}^{0.03}$ \\
\hline 35 & HV 2281 & 301.56 & 984. 16. & $0.90_{0.00}^{0.10}-0.10_{0.10}^{0.00}$ & 203.39. & 504.469 & $0.85_{0.00}^{0.00}-0.15_{0.00}^{0.00}$ \\
\hline 36 & HV 2444 & 323.227 & 505.56 & $0.50_{0.30}^{0.40}-0.50_{0.40}^{0.30}$ & 575.25. & 733. 126. & $0.81_{0.20}^{0.10}-0.19_{0.10}^{0.20}$ \\
\hline 37 & HV 2522 & 218.67 & 916.85 & $0.60_{0.40}^{0.30}-0.40_{0.30}^{0.40}$ & 304..$_{4}^{11}$. & 714.34 & $0.87_{0.10}^{0.04}-0.13_{0.04}^{0.10}$ \\
\hline 38 & HV 2862 & 117. 935. & 613.104: & $0.90_{0.10}^{0.00}-0.10_{0.00}^{0.10}$ & 200.0 & 500.0 & $0.90_{0.00}^{8.00}-0.10_{0.00}^{0.84}$ \\
\hline 39 & HV 5829 & 250.66 & 783. 136 & $0.90_{0.10}^{0.10}-0.10_{0.00}^{0.10}$ & 203.19 & 656.74 & $0.92_{0.03}^{0.03}-0.08_{0.03}^{0.03}$ \\
\hline 40 & HV 915 & 230.105. & 783. 1190 & $0.90_{0.00}^{0.00}-0.10_{0.00}^{0.10}$ & 520.8213 & 719. 101. & $0.75_{054}^{0.17}-0.25_{0.17}^{0.54}$ \\
\hline 41 & J044458.18-703522.8 & 209.96 & $452.91:$ & $0.90_{0.00}^{0.00}-0.10_{0.00}^{0.00}$ & $431.700^{20}$ & 681.33. & $0.91_{0.01}^{0.01}-0.09_{0.01}^{0.01}$ \\
\hline 42 & J045242.93-704737.4 & 243.139. & 712.204. & $0.80_{0.20}^{0.10}-0.20_{0.10}^{0.20}$ & 196.4. & 695.5. & $0.95_{0.02}^{0.01}-0.05_{0.01}^{0.01}$ \\
\hline 43 & J050143.18-694048.7 & 100.0 & 1000. & $0.00^{0.00}-1.00^{0.00}$ & 400.0 & $1000^{0}$ & $0.90^{0.000}-0.10^{0.000}$ \\
\hline 44 & J051159.11-692532.8 & 204.186. & 709.120. & $0.90_{0.10}^{0.00}-0.10_{0.00}^{0.10}$ & 313.90 & 806.120. & $0.93^{8.01}-0.07^{0.02}$ \\
\hline 45 & J051333.74-663419.1 & 226.684. & 990.10. & $0.10_{0.10}^{0.10}-0.90^{0.10}$ & $625.82:$ & 999.1: & $0.90_{000}^{8.84}-0.10^{8.804}$ \\
\hline 46 & J052220.87-655551.6 & 200.0 & $986.89:$ & $0.90_{0.00}^{0.10}-0.10_{0.00}^{0.000}$ & 200. & 1000.0 & $0.97^{0.00}-0.03^{0.000}$ \\
\hline 47 & J053605.56-695802.9 & 183.50. & 812.117. & $0.90^{0.00}-0.10^{0.10}$ & 123.166. & 815.152. & $0.98^{0.01}-0.02^{0.07}$ \\
\hline 48 & J054312.52-683356.9 & 200.0. & $\begin{array}{l}950.50 . \\
90 .\end{array}$ & $0.90_{0.10}^{0.10}-0.10_{0.00}^{0.00}$ & 400.0. & 996.45 & $0.94_{0.02}^{0.00}-0.06_{0.00}^{0.01}$ \\
\hline 49 & МАСНО 78.6698.38 & 125.76. & $533.234:$ & $0.90_{0.00}^{0.100}-0.10_{0.00}^{0.000}$ & 151.50 & 701. & $0.94_{0.04}^{0.01}-0.06_{0.01}^{0.04}$ \\
\hline 50 & MACHO 82840515 & $208.98:$ & 501. $1.00:$ & $0.90_{0.10}^{0.00}-0.10_{0.00}^{8.00}$ & 300.0 & 500.0 & $0.82_{0.02}^{0.84}-0.18_{0.03}^{0.81}$ \\
\hline 51 & MSX 949 & 100.0 & 200.0 & $0.70_{0.10}^{0.10}-0.30_{0.00}^{8.10}$ & 200.0 & 500.0 & $0.95_{0.00}^{0.80}-0.05_{0.00}^{0.80}$ \\
\hline 52 & NGC 1805SAGEIRS 1 & 242.88 & 582.243. & $0.80_{0.40}^{0.10}-0.20_{0.10}^{0.40}$ & 289.20. & 786.30. & $0.89_{0.06}^{0.00}-0.11_{0.01}^{0.06}$ \\
\hline 53 & SAGE 050830 & & 418.84 & $0.80_{0.10}^{0.40}-0.20_{0.10}^{0.00}$ & 248.320. & 877.69 & $0.89^{0.05}-0.12^{0.24}$ \\
\hline 54 & SAGE 051453 & & 376.24 & $0.90_{0.10}^{0.00}-0.10^{0.10}$ & 200. & 412. & $0.90_{0.02}^{0.02}-0.10_{0}^{0.13}$ \\
\hline 55 & SAGE 052707 & & $681.120:$ & $0.90_{0.10}^{8.10}-0.10_{0.00}^{8.00}$ & $298 .{ }^{2}$ & 998.2. & $0.90_{0.00}^{8.073}-0.10^{0.80}$ \\
\hline 56 & SAGE 052747 & & 524. & $0.90_{0.10}^{0.10}-0.10_{0.10}^{0.10}$ & 331.70 & 990.10 & $0.88^{0.02}-0.12^{0.03}$ \\
\hline 57 & SAGE 054310 & 197.3. & 465.35. & $0.90_{0.00}^{0.00}-0.10_{0.00}^{0.00}$ & 200.0. & 430.710 . & $0.95_{0.00}^{0.00}-0.05_{0.00}^{0.00}$ \\
\hline
\end{tabular}

Notes. Listed the dust and continuum temperatures and their relative fractions. 
C. Gielen et al.: Silicate features in Galactic and extragalactic post-AGB disks

Table A.2. Best fit parameters deduced from our full spectral fitting.

\begin{tabular}{|c|c|c|c|c|}
\hline $\mathrm{N}^{\circ}$ & $\begin{array}{c}\text { MgOlivine } \\
\text { Small - Medium - Large }\end{array}$ & $\begin{array}{c}\text { MgPyroxene } \\
\text { Small - Medium - Large }\end{array}$ & $\begin{array}{c}\text { MgFeOlivine } \\
\text { Small - Medium - Large }\end{array}$ & $\begin{array}{c}\text { MgFePyroxene } \\
\text { Small - Medium - Large }\end{array}$ \\
\hline 1 & $0.00_{0.00}^{0.00}-0.00_{0.00}^{0.00}-0.00_{0.00}^{0.00}$ & $0.05_{0.05}^{0.00}-0.00_{0.00}^{0.00}-0.00_{0.00}^{0.00}$ & $44.26_{4.57}^{6.75}-0.00_{0.00}^{0.00}-34.12_{13.02}^{5.73}$ & $0.00_{0.00}^{0.00}-0.00_{0.00}^{0.00}-0.00_{0.00}^{0.00}$ \\
\hline 2 & $1.24_{1.08}^{3.32}-0.00_{0.00}^{0.00}-14.39_{5.46}^{3.41}$ & $9.29_{1.89}^{0.05}-0.00_{0.00}^{0.00}-3.31_{2.94}^{0.00}$ & $0.00_{0.00}^{0.00}-0.00_{0.00}^{0.00}-0.62_{0.62}^{6.21}$ & $0.00_{0.00}^{0.00}-0.00_{0.00}^{0.00}-44.60^{5.33}$ \\
\hline 3 & $0.00_{0.00}^{0.00}-0.00_{0.00}^{0.00}-16.25_{257}^{2.46}$ & $4.75_{0.83}^{0.92}-0.00_{0.00}^{0.00}-11.16_{5.11}^{4.11}$ & $0.00_{0.00}^{0.00}-0.00_{0.00}^{0.00}-0.01_{0.02}^{0.02}$ & $0.00_{0.00}^{0.00}-0.00_{0.00}^{0.00}-49.23_{485}^{6.07}$ \\
\hline 4 & $0.00_{0.00}^{0.00}-0.00_{0.00}^{0.00}-0.00_{0.00}^{0.00}$ & $36.80_{3.63}^{1.40}-0.00_{0.00}^{0.00}-0.00_{0.00}^{8.00}$ & $0.00_{0.00}^{8.00}-0.00_{0.00}^{8.00}-0.00_{0.00}^{0.00}$ & $0.00_{0.00}^{0.00}-0.00_{0.00}^{0.00}-0.00_{0.00}^{0.00}$ \\
\hline 5 & $0.00_{0.00}^{0.00}-0.00_{0.00}^{0.00}-0.00_{0.00}^{0.00}$ & $11.06_{2.61}^{8.99}-0.00_{0.00}^{0.00}-1.18_{1.15}^{3.10}$ & $17.15_{5.15}^{7.62}-0.00_{0.00}^{0.00}-15.48_{7.14}^{5.025}$ & $0.00_{0.00}^{0.00}-0.00_{0.00}^{0.00}-0.00_{0.00}^{0.00}$ \\
\hline 6 & $34.95_{26.48}^{11.28}-0.00_{0.00}^{0.00}-0.00_{0.00}^{0.00}$ & $4.06_{3.88}^{9.01}-0.00_{0.00}^{0.00}-0.00_{0.00}^{0.000}$ & $0.00_{0,00}^{0.00}-0.00_{0.00}^{0.00}-0.00_{0,00}^{0.00}$ & $0.00_{0.00}^{0.00}-0.00_{0.00}^{0.00}-0.00_{0.00}^{0.00}$ \\
\hline 7 & $2.71_{2.58}^{5.048}-0.00_{0.00}^{0.000}-10.13_{9.95}^{6.80}$ & $0.42_{0.41}^{1.82}-0.00_{0.00}^{0.00}-0.18_{0.18}^{3.08}$ & $19.04_{19.29}^{12.34}-0.00_{0.00}^{0.00}-8.80_{8.80}^{14.46}$ & $0.00_{0.00}^{0.00}-0.00_{0.00}^{0.00}-0.00_{0.00}^{0.00}$ \\
\hline 8 & $0.00_{0.00}^{0.00}-0.00_{0.00}^{0.00}-0.00_{0.00}^{0.00}$ & $61.88_{29.71}^{10.53}-0.00_{0.00}^{0.00}-0.35_{0.35}^{11.55}$ & $10.11_{10.18}^{36.34}-0.00_{0.00}^{0.00}-0.00_{0.00}^{0.00}$ & $0.00_{0.00}^{0.00}-0.00_{0.00}^{0.00}-0.00_{0.00}^{0.00}$ \\
\hline 9 & $0.00_{0.00}^{0.00}-0.00_{0.00}^{0.00}-17.23_{5.91}^{2.90}$ & $21.53_{0.94}^{1.05}-0.00_{0.00}^{0.00}-6.72_{3.20}^{0.37}$ & $1.89_{1.44}^{1.78}-0.00_{0.00}^{0.000}-18.43_{3.13}^{6.89}$ & $0.00_{0.00}^{0.00}-0.00_{0.00}^{0.00}-0.00_{0.00}^{0.00}$ \\
\hline 10 & $0.00_{0.00}^{0.00}-0.00_{0.00}^{0.00}-12.05_{9.01}^{4.80}$ & $10.29_{4.30}^{1.57}-0.00_{0.00}^{0.00}-16.91_{5.12}^{8.84}$ & $0.11_{0.11}^{4.84}-12.48_{9.12}^{5.73}-21.69_{5.87}^{319.58}$ & $0.00_{0.00}^{0.00}-0.00_{0.00}^{0.00}-2.85_{2.61}^{5.09}$ \\
\hline 11 & $0.00_{0.00}^{0.00}-0.00_{0.00}^{0.00}-5.66_{5.66}^{19.27}$ & $0.65_{0.60}^{3.111}-0.00_{0.00}^{0.00}-22.03_{4.83}^{4.15}$ & $0.00_{0.00}^{0.34}-0.00_{0.00}^{0.00}-48.58_{30.81}^{10.07}$ & $0.00_{0.00}^{0.00}-0.00_{0.00}^{0.00}-3.06_{3.07}^{15.32}$ \\
\hline 12 & $24.17_{577}^{3.02}-0.79_{079}^{3.98}-0.28_{0.28}^{2.49}$ & $0.01_{0.01}^{0.00}-13.60_{642}^{4.15}-8.45_{502}^{6.839}$ & $24.29_{353}^{4.79}-1.14_{1.5}^{4.54}-27.00_{3.73}^{3.34}$ & $0.00_{0.00}^{0.00}-0.00_{0.00}^{0.00}-0.00_{0.00}^{0.00}$ \\
\hline 13 & $6.79_{462}^{8.22}-0.84_{0.84}^{7.81}-1.71_{169}^{8.93}$ & $20.96_{430}^{3.29}-0.00_{0.00}^{8.00}-0.03_{0.03}^{1.29}$ & $17.07_{478}^{6.63}-0.16_{0.16}^{14.54}-22.17_{1291}^{3.655}$ & $0.03_{0.03}^{2.32}-0.00_{0.00}^{0.00}-0.00_{0.00}^{0.00}$ \\
\hline 14 & $0.00_{0.00}^{0.02}-0.00_{0.00}^{0.00}-0.00_{0.00}^{0.00}$ & $0.00_{0.00}^{0.00}-0.00_{0.00}^{0.00}-0.00_{0.00}^{0.00}$ & $0.00_{0.00}^{0.00}-0.00_{000}^{0.00}-83.47_{0.88}^{0.75}$ & $0.00_{0.00}^{0.00}-0.00_{0.00}^{0.00}-0.00_{0.00}^{0.00}$ \\
\hline 15 & $0.00_{0.00}^{0.00}-0.00_{0.00}^{0.00}-0.00_{0.00}^{0.00}$ & $1.64_{1.4}^{14.18}-0.00_{0.00}^{0.00}-0.00_{0.00}^{0.00}$ & $66.37_{989}^{1.96}-0.00_{0.00}^{0.00}-0.1$ & $0.07_{0.07}^{3.02}-0.00_{0.00}^{0.00}-0.00_{0.00}^{0.00}$ \\
\hline 16 & $0.00_{0.00}^{0.00}-0.00_{0.00}^{0.00}-23.00_{6.45}^{3.41}$ & $5.21_{071}^{1.64}-0.00_{0.00}^{0.00}-0.00_{0.00}^{0.00}$ & $0.00^{0.000}-0.00_{0.00}^{0.00}-41.98_{2}^{0.335}$ & $0.00_{0.00}^{0.07}-0.00_{0.00}^{0.00}-0.00_{0.00}^{0.00}$ \\
\hline 17 & $0.00_{0.00}^{0.00}-0.00_{0.00}^{0.00}-19.60_{10.76}^{6.83}$ & $3.29^{5.27}-0.02-00_{0.00}^{0.000}-10.87_{10.38}^{0.00}$ & $1.21_{1.17}^{3.42}-0.00_{0.00}^{0.00}-7.86_{6.97}^{28.56}$ & $0.00_{0.00}^{0.00}-0.00_{0.00}^{0.00}-1.32_{1.31}^{0.00}$ \\
\hline 18 & $0.92_{0.88}^{2.77}-0.00_{0.00}^{0.00}-0.05_{0.05}^{3.11 .16}$ & $8.28_{2.02}^{1.62}-0.00_{0.00}^{0.00}-0.00_{0.00}^{0.00}$ & $38.44_{2}^{3.41}-0.00_{0.00}^{0.00}-0.00_{0.00}^{0.00}$ & $0.00_{0.00}^{0.00}-0.00_{0.00}^{0.00}-0.00_{0.00}^{0.00}$ \\
\hline 19 & $0.00_{0.00}^{0.00}-0.00_{0.00}^{0.00}-14.95_{3.36}^{3.03}$ & $6.52_{1.17}^{1.116}-0.00_{0.00}^{0.00}-0.00_{0.00}^{0.00}$ & $24.04_{2.70}^{3.95}-0.02_{0.02}^{0.00}-17.42_{322}^{3.40}$ & $0.00_{0.00}^{0.00}-0.00_{0.00}^{0.00}-0.00_{0.00}^{0.00}$ \\
\hline 20 & $20.41_{632}^{2.77}-0.00_{0.00}^{0.00}-0.00_{0.00}^{0.00}$ & $21.91_{634}^{1.86}-0.69_{069}^{11.80}-0.00_{0.00}^{0.00}$ & $36.06_{544}^{4.86}-0.25_{0.25}^{20.56}-0.51^{20.51}$ & $0.00_{0.00}^{0.00}-0.00_{0.00}^{0.00}-0.00_{0.00}^{0.00}$ \\
\hline 21 & $0.00_{0.00}^{0.00}-0.18_{0.18}^{2.12}-14.92_{14.64}^{32.27}$ & $0.00_{0.00}^{0.00}-0.00_{0.00}^{0.00}-1.61_{159}^{8.0 .00}$ & $22.95_{1424}^{7.74}-0.00_{0.00}^{0.00}-26.96_{24.87}^{13.436}$ & $0.00_{0.00}^{0.00}-0.00_{0.00}^{0.00}-0.00_{0.00}^{0.00}$ \\
\hline 22 & $0.43_{0.44}^{3.40}-0.00_{0.00}^{0.00}-6.47_{5.14}^{1.06}$ & $6.55_{2,12}^{1.00}-0.35_{0.35}^{5.38}-5.98_{4.17}^{4.68}$ & $0.90_{0.90}^{4.944}-0.00_{0.00}^{0.00}-52.59_{585}^{5.3478}$ & $0.00_{0.00}^{0.00}-0.00_{0.00}^{0.00}-0.34_{0.34}^{5.00}$ \\
\hline 23 & $3.57_{2.61}^{2.24}-0.00_{0.00}^{0.00}-0.35_{0.35}^{5.94}$ & $14.90_{2.41}^{2.59}-0.00_{0.00}^{0.00}-0.00_{0.00}^{0.00}$ & $6.19_{3.63}^{4.39}-0.00_{0.00}^{0.00}-40.44_{1.67}^{1.95}$ & $0.00_{0.00}^{0.00}-0.00_{0.00}^{0.00}-0.00_{0.00}^{0.00}$ \\
\hline 24 & $0.56_{0.55}^{4.141}-0.00_{0.00}^{0.00}-47.31_{3.41}^{2.76}$ & $7.82_{1.52}^{1.09}-0.00_{0.00}^{0.00}-3.83_{2.98}^{3.22}$ & $0.00_{0.00}^{0.00}-0.00_{0.00}^{0.00}-0.33_{0.33}^{12.32}$ & $0.00_{0.00}^{0.00}-0.00_{0.00}^{0.00}-0.00_{0.00}^{0.00}$ \\
\hline 25 & $5.12_{4.82}^{6.22}-0.00_{0.00}^{0.00}-0.00_{0.00}^{0.001}$ & $1.57_{1.50}^{3.46}-0.00_{0.00}^{0.00}-0.00_{0.00}^{0.00}$ & $49.34_{6.66}^{6.75}-0.00_{0.00}^{0.00}-10.66_{10.57}^{14.52}$ & $0.01_{0.01}^{0.73}-0.00_{0.00}^{0.00}-1.44_{1.43}^{9.16}$ \\
\hline 26 & $0.00_{000}^{0.00}-0.00_{000}^{0.000}-2.86_{2.68}^{5.604}$ & $4.32_{2.02}^{1.30}-0.00_{0.00}^{0.00}-0.00_{0.00}^{0.00}$ & $4.14_{301}^{5.00}-0.29_{022}^{26.96}-38.25_{780}^{4.60}$ & $0.00_{000}^{0.00}-0.00_{000}^{0.00}-0.00_{0.00}^{0.00}$ \\
\hline 27 & $0.00_{0.00}^{0.00}-0.00_{0.00}^{0.00}-20.26_{7.02}^{4.30}$ & $0.01_{0.01}^{0.00}-0.00_{0.00}^{8.00}-5.61_{2.40}^{2.65}$ & $0.00_{0.00}^{0.00}-0.00_{0.00}^{0.00}-6.28_{4.89}^{11.78}$ & $0.00_{0.00}^{0.00}-0.00_{0.00}^{0.00}-29.29_{6.57}^{4.04}$ \\
\hline 28 & $0.00_{0.00}^{0.00}-0.00_{0.00}^{0.00}-10.32_{6.89}^{8.18}$ & $0.67_{0.67}^{3.39}-0.00_{0.00}^{0.00}-7.62_{4.37}^{5.90}$ & $25.20_{4.23}^{3.63}-4.95_{4.70}^{8.99}-7.79_{7.00}^{13.98}$ & $0.00_{0.00}^{0.00}-0.00_{0.00}^{0.00}-4.76_{4.54}^{8.42}$ \\
\hline 29 & $0.00_{0.00}^{0.00}-0.00_{0.00}^{0.00}-18.98_{13.72}^{14.04}$ & $0.04_{0.04}^{0.67}-0.00_{0.00}^{0.00}-0.00_{0.00}^{0.00}$ & $8.32_{8,29}^{11.53}-0.06_{0.06}^{1.73}-52.42_{20.47}^{16.56}$ & $0.00_{0.00}^{0.00}-0.00_{0.00}^{0.00}-0.00_{0.00}^{0.04}$ \\
\hline 30 & $0.00_{0.00}^{0.00}-0.00_{0.00}^{0.00}-7.67_{5.27}^{6.21^{2}}$ & $10.14_{2.08}^{0.70}-0.00_{0.00}^{0.00}-0.00_{0.00}^{0.00}$ & $0.00_{0.00}^{0.00}-0.00_{0.00}^{0.00}-36.42_{7.28}^{19.14}$ & $0.00_{0.00}^{8.00}-0.00_{0.00}^{8.00}-4.61_{3.32}^{2.50}$ \\
\hline 31 & $0.00_{0.00}^{0.00}-0.00_{0.00}^{0.00}-79.45_{11.38}^{5.43}$ & $0.00_{0.00}^{0.00}-0.00_{0.00}^{0.00}-0.00_{0.00}^{0.00}$ & $0.00_{0.00}^{0.00}-0.00_{0.00}^{0.00}-2.56_{2.46}^{9.18}$ & $0.00_{0.00}^{8.00}-0.00_{0.00}^{0.00}-0.00_{0.00}^{8.00}$ \\
\hline 32 & $0.00_{0.00}^{0.00}-0.00_{0.00}^{0.00}-8.47_{4.70}^{4.59}$ & $0.14_{0.14}^{1.07}-0.00_{0.00}^{0.00}-20.70_{6.47}^{5.91}$ & $0.00_{0.00}^{0.00}-0.00_{0.00}^{0.00}-0.24_{0.24}^{4.08}$ & $0.00_{0.00}^{0.00}-0.00_{0.00}^{0.00}-47.64_{7.17}^{8.11}$ \\
\hline 33 & $2.28_{2.04}^{4.14}-0.07_{0.07}^{3.41}-5.40_{3.33}^{3.30}$ & $6.36_{1.06}^{1.113}-0.00_{0.00}^{0.00}-26.60_{2.71}^{2.86}$ & $0.00_{0.00}^{0.00}-0.00_{0.00}^{0.00}-0.00_{0.00}^{0.00}$ & $0.00_{0.00}^{0.00}-0.00_{0.00}^{0.00}-0.00_{0.00}^{0.00}$ \\
\hline 34 & $0.00_{0.00}^{0.00}-0.00_{0.00}^{0.00}-0.01_{0.01}^{0.00}$ & $7.20_{4.20}^{4.04}-0.00_{0.00}^{0.00}-10.02_{8.77}^{15.05}$ & $18.35_{11.11}^{11.41}-2.03_{1.99}^{12.79}-9.83_{8.75}^{13.55}$ & $0.00_{0.00}^{0.00}-0.00_{0.00}^{0.00}-0.12_{0.12}^{9.23}$ \\
\hline 35 & $0.01_{0.01}^{0.00}-0.00_{0.00}^{0.00}-0.00_{0.00}^{0.00}$ & $1.18_{1.16}^{5.47}-0.43_{0.43}^{6.74}-6.50_{6.00}^{11.11}$ & $20.83_{1049}^{10.49}-1.86_{1.86}^{13.23}-1.04_{1.04}^{81.42}$ & $9.47_{8.10}^{10.78}-1.04_{1.05}^{14.14}-2.58_{2.53}^{9.53}$ \\
\hline 36 & $2.28_{206}^{6.41}-0.28_{028}^{5.00}-0.00_{0.00}^{0.00}$ & $0.00_{0.00}^{0.00}-0.37_{037}^{0.03}-52.93_{953}^{8.9 .96}$ & $0.54_{054}^{8.36}-0.00_{0.00}^{0.00}-7.49_{611}^{11.67}$ & $0.00_{0.00}^{0.00}-0.00_{0.00}^{0.00}-0.00_{0.00}^{0.00}$ \\
\hline 37 & $4.23_{4.06}^{21.06}-0.00_{0.00}^{0.00}-0.10_{0.10}^{4.27}$ & $6.05_{500}^{5.65}-0.10_{0.10}^{4.00}-1.38_{138}^{12.17}$ & $17.53_{1372}^{14.64}-1.21_{121}^{13.48}-4.61_{457}^{13.99}$ & $0.09_{0.09}^{3.770}-0.20_{020}^{6.82}-25.25_{1898}^{36.91}$ \\
\hline 38 & $0.00_{0.00}^{0.00}-0.00^{0.000}-0.49_{0.48}^{7.59}$ & $0.00_{0.00}^{6.00}-0.00_{0.00}^{6.00}-2.66^{6.42}$ & $0.00_{0.00}^{0.00}-0.00_{000}^{0.000}-16.68_{745}^{8.27}$ & $0.00_{0.00}^{0.00}-0.00_{0.00}^{0.00}-63.03_{876}^{7.77}$ \\
\hline 39 & $0.00_{0.00}^{0.00}-0.00_{0.00}^{0.00}-9.69_{8.62}^{0.78}$ & $3.76_{3.58}^{7.100}-0.03_{0.03}^{0.00}-17.81_{12.93}^{2.59 .96}$ & $1.89^{8.67}-0.40_{040}^{17.45}-18.43^{16.450}$ & $0.00^{0.00}-0.00_{0.00}^{0.00}-27.97_{2470}^{30.26}$ \\
\hline 40 & $6.12_{5.25}^{10.41}-0.61_{0.62}^{18.84}-28.64_{15.94}^{11.62}$ & $0.21_{0.21}^{2.09}-0.00_{0.00}^{0.00}-0.00_{0.00}^{0.0093}$ & $0.00_{0.00}^{0.00}-0.00_{0.40}^{0.00}-0.00_{0.00}^{0.00}$ & $0.00_{0.00}^{0.00}-0.00_{0.00}^{0.00}-0.00_{0.00}^{0.00}$ \\
\hline 41 & $0.04_{004}^{2.22}-0.00_{0.00}^{0.00}-0.66_{0.66}^{7.67}$ & $7.21_{586}^{5.221}-0.10_{0.10}^{0.00}-35.09_{1160}^{9.80}$ & $12.16_{805}^{9.49}-0.00^{0.00}-0.12^{10.512}$ & $0.00_{0.00}^{0.00}-0.08^{5.82}-5.19^{13.38}$ \\
\hline 42 & $0.00_{0.00}^{0.004}-0.00_{0.00}^{0.00}-11.09_{8.23}^{0.66}$ & $0.32_{0.32}^{9.42}-0.15_{0.16}^{0.00}-32.89_{8.83}^{7.30}$ & $0.00_{0.00}^{0.32}-0.02_{0.02}^{0.00}-17.20_{11.06}^{11.72}$ & $0.02_{0.02}^{0.00}-0.00_{0.00}^{0.00}-12.24_{8.19}^{5.53}$ \\
\hline 43 & $0.00_{0.00}^{0.00}-0.02_{0.02}^{2.18}-46.94_{6.01}^{8.01}$ & $0.00_{0.00}^{0.00}-0.00_{0.00}^{0.00}-2.12_{2.24}^{6.24}$ & $0.00_{0.00}^{0.00}-0.00_{0.00}^{0.00}-2.51_{2.40}^{8.3 .06}$ & $0.00_{0.00}^{0.00}-0.10_{0.10}^{4.43}-26.29_{756}^{5.195}$ \\
\hline 44 & $0.02_{0.02}^{0.00}-0.01_{0.01}^{0.00}-2.63_{2.53}^{10.05}$ & $13.38_{3.55}^{4.23}-1.23_{1.24}^{100}-11.41_{8.03}^{8.05}$ & $2.28_{.18}^{6.87}-10.68_{9.80}^{13.90}-34.38_{16.03}^{110.63}$ & $0.01_{0.01}^{0.00}-0.05_{0.05}^{0.00}-0.00_{0.00}^{0.00}$ \\
\hline 45 & $0.00_{0.00}^{0.002}-24.43_{12.09}^{12.61}-30.16_{18.98}^{15.65}$ & $0.00_{0.00}^{0.00}-0.00_{0.00}^{1.00}-0.00_{0.00}^{0.00}$ & $0.00_{0.00}^{0.00}-0.00_{0.00}^{0.00}-43.97_{12.83}^{1.00}$ & $0.00_{0.00}^{0.00}-0.00_{0.00}^{0.05}-0.00_{0.00}^{0.000}$ \\
\hline 46 & $0.03_{0.03}^{1.63}-0.00_{0.00}^{0.00}-0.00_{0.00}^{0.00}$ & $17.13_{4.41}^{4.70}-33.24_{11.13}^{8.62}-5.30_{4.91}^{9.62}$ & $0.00_{0.00}^{0.00}-0.00_{0.00}^{0.00}-0.00_{0.00}^{0.00}$ & $0.00_{0.00}^{0.00}-0.00_{0.00}^{0.00}-0.00_{0.00}^{0.000}$ \\
\hline 47 & $2.08_{1.65}^{0.06}-0.00_{0,00}^{0.00}-1.46_{1.46}^{1.140}$ & $0.55_{0.55}^{2.31}-0.00_{0.00}^{0.00}-26.40_{4.21}^{4.5 .501}$ & $0.00_{0.00}^{0.000}-0.00_{0,00}^{0.00}-41.92_{15,60}^{7.90}$ & $0.00_{0.00}^{0.00}-0.00_{0.00}^{0.00}-0.38_{0.38}^{8.40}$ \\
\hline 48 & $0.93_{0.93}^{5.18}-0.22_{0.22}^{4.06}-0.81_{0.81}^{8.74}$ & $0.13_{0.13}^{2.43}-0.38_{0.38}^{3.60}-2.42_{2.42}^{11.6 \frac{1}{3}}$ & $0.00_{0.00}^{0.00}-0.00_{0.00}^{0.00}-5.76_{5,41}^{14.43}$ & $0.00_{0.00}^{0.00}-0.00_{0.00}^{0.00}-32.11_{14.78}^{15.20}$ \\
\hline 49 & $0.96_{0.96}^{14.89}-0.00_{0.00}^{0.00}-0.00_{0.00}^{0.00}$ & $24.35_{5.46}^{11.53}-0.51_{0.51}^{16.67}-1.10_{1.10}^{28.26}$ & $0.00_{0.00}^{0.00}-0.00_{0.00}^{0.00}-36.95_{31.06}^{29.60}$ & $0.00_{0.00}^{0.00}-0.00_{0.00}^{0.00}-0.00_{0.00}^{0.00}$ \\
\hline 50 & $1.70_{1.68}^{10.63}-0.00_{0.00}^{0.00}-15.44_{13.59}^{13.45}$ & $17.59_{6.25}^{5.40}-0.90_{0.90}^{10.62}-5.66_{5.36}^{15.67}$ & $4.99_{4.69}^{9.62}-0.00_{0.00}^{0.00}-0.77_{0.77}^{9.36}$ & $0.28_{0.28}^{23.77}-0.00_{0.00}^{0.00}-0.71_{0.71}^{15.36}$ \\
\hline 51 & $0.00_{0.00}^{0.00}-0.00_{0.00}^{0.00}-0.01_{0.01}^{0.00}$ & $21.94_{2.72}^{2.21}-0.00_{0.00}^{0.00}-0.54_{0.54}^{12.00}$ & $0.00_{0.00}^{0.00}-0.00_{0.00}^{0.00}-56.16_{4.07}^{2.92}$ & $0.00_{0.00}^{0.00}-0.00_{0.00}^{0.00}-0.00_{0.00}^{0.00}$ \\
\hline 52 & $3.68_{3.47}^{8.63}-4.39_{4.28}^{10.09}-0.88_{0.89}^{7.18}$ & $17.63_{8.55}^{8.46}-9.19_{8.55}^{15.60}-5.39_{5.05}^{11.11}$ & $2.49_{2.41}^{8.27}-0.53_{0.54}^{7.85}-1.08_{1.07}^{9.51}$ & $5.27_{5.13}^{14.71}-3.65_{3.56}^{11.34}-15.34_{14.18}^{26.80}$ \\
\hline 53 & $0.00_{0.00}^{0.00}-0.00_{0.00}^{0.00}-0.00_{0.00}^{0.00}$ & $22.95_{2.05}^{2.69}-0.00_{0.00}^{0.00}-8.08_{5.72}^{6.047}$ & $0.05_{0.05}^{0.00}-10.41_{6.91}^{7.33}-6.98_{5.56}^{6.77}$ & $0.06_{0.06}^{2.17}-0.00_{0.00}^{0.00}-0.01_{0.01}^{0.00}$ \\
\hline 54 & $0.00_{0.00}^{0.00}-0.00_{0.00}^{0.00}-0.00_{0.00}^{0.00}$ & $24.94_{17.43}^{6.47}-0.00_{0.00}^{0.00}-4.44_{4.44}^{31.90}$ & $0.00_{0.00}^{0.00}-0.00_{0.00}^{0.00}-7.72_{7.77}^{43.30}$ & $0.00_{0.00}^{0.00}-0.00_{0.00}^{0.00}-0.33_{0.33}^{9.61}$ \\
\hline 55 & $40.18_{563}^{5.98}-0.00_{0.00}^{0.00}-0.03_{0.03}^{0.00}$ & $4.85_{2.89}^{3.48}-1.96_{190}^{4.750}-4.32_{3.59}^{6.57}$ & $1.26_{126}^{9.05}-0.00_{0.00}^{0.00}-17.98_{6.74}^{6.01}$ & $0.00_{0.00}^{0.00}-0.03_{0.03}^{0.00}-0.12_{0.12}^{3.99}$ \\
\hline 56 & $2.03_{2.00}^{12.71^{3}}-0.00_{0.00}^{0.00}-33.82_{13.76}^{0.093}$ & $13.31_{6.93}^{5.13}-0.00_{0.00}^{0.00}-8.85_{777}^{9.82}$ & $9.80_{6.56}^{14.84}-0.00_{0.00}^{0.00}-9.71_{7.77}^{10.74}$ & $0.25_{0.25}^{3.42}-0.00_{0.00}^{0.00}-1.02_{1.02}^{14.62}$ \\
\hline 57 & $0.00_{0.00}^{0.00}-0.00_{0.00}^{0.00}-0.00_{0.00}^{0.00}$ & $18.84_{3.40}^{0.97}-0.00_{0.00}^{0.00}-2.68_{2.70}^{13.40}$ & $3.20_{3.05}^{6.98}-0.00_{0.00}^{0.00}-9.92_{9.92}^{25.50}$ & $0.03_{0.03}^{0.00}-0.00_{0.00}^{0.00}-0.00_{0.00}^{0.00}$ \\
\hline
\end{tabular}

Notes. The abundances of small, medium and large grains of the various dust species are given as fractions of the total mass, excluding the dust responsible for the continuum emission. 
Table A.3. Best fit parameters deduced from our full spectral fitting.

\begin{tabular}{|c|c|c|c|c|}
\hline $\mathrm{N}^{\circ}$ & $\begin{array}{c}\text { Silica } \\
\text { Small - Medium - Large }\end{array}$ & $\begin{array}{c}\text { Forsterite } \\
\text { Small - Medium - Large }\end{array}$ & $\begin{array}{c}\text { Enstatite } \\
\text { Small - Medium - Large }\end{array}$ & Continuum \\
\hline 1 & $0.00_{0.00}^{0.00}-0.00_{0.00}^{0.00}-0.00_{0.00}^{0.00}$ & $8.66_{2.37}^{3.64}-3.85_{2.44}^{2.56}-0.05_{005}^{4.58}$ & $0.01_{0.01}^{1.19}-0.00_{0.00}^{0.00}-9.00_{318}^{3.30}$ & $52.84_{0.85}^{4.99}$ \\
\hline 2 & $0.00_{0.00}^{0.00}-0.00_{0.00}^{0.00}-3.44_{0.84}^{2.00}$ & $1.44_{056}^{0.39}-18.35_{1.10}^{2.77}-0.00_{0.00}^{0.00}$ & $0.02_{0.02}^{0.01}-0.00_{0.00}^{0.00}-3.31_{149}^{2.18}$ & $77.81_{031}^{1.85}$ \\
\hline 3 & $0.00^{0.11}-0.22^{0.75}-3.55_{100}^{0.84}$ & $0.50_{030}^{0.37}-12.61_{089}^{0.106}-0.00_{0.00}^{0.00}$ & $0.37^{0.40}-0.00^{0.00}-1.33^{1.33}$ & $74.46_{0.20}^{0.21}$ \\
\hline 4 & $0.01_{0.06}^{0.36}-14.03_{186}^{0.96}-1.41_{127}^{3.11}$ & $13.45^{1.38}-8.92^{4.67}-24.74_{466}^{4.29}$ & $0.00^{0.00}-0.00^{0.00}-0.64^{3.01} .30$ & $67.56^{0.37}$ \\
\hline 5 & $0.27_{020}^{0.25}-0.00_{0.00}^{0.00}-0.00_{0.00}^{0.00}$ & $4.99^{0.47}-19.90^{1.59}-0.00^{0.000}$ & $4.77_{0.68}^{0.87}-0.00_{0.00}^{0.00}-25.19^{1.94}$ & $69.00^{0.68}$ \\
\hline 6 & $0.25^{1.24}-0.00^{0.00}-0.17^{1.33}$ & $6.07^{1.10}-12.27^{2.88}-17.12^{9.58}$ & $2.90^{2.15}-2.48^{3.60}-19.74^{7.7176}$ & $79.35^{0.64}$ \\
\hline 7 & $0.00_{0.00}^{0.00}-0.00_{0.00}^{0.00}-0.15_{0.15}^{0.17}$ & $4.63_{051}^{1.53}-9.81_{569}^{3.53}-23.91^{18.41}$ & $0.50_{049}^{1.266}-0.00_{0.00}^{0.00}-19.72_{4.34}^{2.143}$ & $82.26_{0.95}^{1.57}$ \\
\hline 8 & $20.14_{3.37}^{2.50}-0.55_{0.55}^{7.15}-0.00_{0.00}^{0.00}$ & $5.16_{3.40}^{3.60}-1.53_{1.49}^{7.41}-0.00_{0.00}^{0.002}$ & $0.00_{0.00}^{0.00}-0.00_{0.00}^{0.00}-0.27_{0.27}^{13.44}$ & $81.87_{168}^{1.95}$ \\
\hline 9 & $0.00_{0.00}^{0.00}-0.00_{0.00}^{0.00}-2.50_{0.49}^{0.049}$ & $3.21_{0.30}^{0.37}-12.70_{0.88}^{0.78}-0.46_{0.45}^{2.57}$ & $0.00_{0.00}^{0.00}-0.00_{0.00}^{0.00}-15.33_{1.16}^{1.113}$ & $70.18_{034}^{0.29}$ \\
\hline 10 & $1.85_{0.71}^{0.30}-0.00_{0.00}^{0.00}-7.29_{0.83}^{2.49}$ & $1.11_{0.70}^{0.44}-12.91_{0.87}^{1.05}-0.00_{0.00}^{0.00}$ & $0.06_{0.06}^{0.46}-0.00_{0.00}^{0.00}-0.41_{0.40}^{1.72}$ & $73.51_{2.02}^{0.84}$ \\
\hline 11 & $0.00_{0.00}^{0.14}-0.00_{0.00}^{0.00}-7.29_{1.26}^{1.30}$ & $0.01_{0.01}^{0.27}-10.21_{1,89}^{2.80}-2.50_{2,09}^{2.80}$ & $0.00_{0.00}^{0.00}-0.00_{0.00}^{0.00}-0.00_{0.00}^{0.22}$ & $79.83_{1.06}^{2.02}$ \\
\hline 12 & $0.00_{0.00}^{8.00}-0.00_{0.00}^{8.00}-0.20_{0.19}^{0.79}$ & $0.01_{0.01}^{0.09}-0.07_{0.06}^{0.26}-0.01_{0.01}^{0.29}$ & $0.00_{0,00}^{0.00}-0.00_{0.00}^{8.00}-0.00_{0,00}^{80.00}$ & $28.11_{3.30}^{1.09}$ \\
\hline 13 & $1.06_{0.40}^{8.34}-0.00_{0.00}^{8.00}-0.44_{0.42}^{8.93}$ & $0.03_{0.04}^{0.43}-12.83_{0.75}^{0.50}-0.42_{0.38}^{0.80}$ & $0.00_{0.00}^{0.00}-0.00_{0,00}^{0.00}-15.47_{056}^{0.58}$ & $74.30_{0.75}^{1.04}$ \\
\hline 14 & $0.04_{0.04}^{8.42}-0.07_{0.07}^{8.45}-0.29_{0.26}^{8.46}$ & $0.00_{0.00}^{0.00}-11.44_{0.33}^{0.28}-0.09_{0.09}^{0.68}$ & $3.12_{0.35}^{0.38}-0.00_{0.00}^{0.00}-1.47_{0.86}^{1.00}$ & $52.14_{0.43}^{0.48}$ \\
\hline 15 & $0.02_{0.02}^{0.67}-0.00_{0.00}^{0.00}-0.00_{0.00}^{8.08}$ & $0.50_{0.48}^{2.26}-5.61_{1,60}^{0.86}-2.43_{1,30}^{1.29}$ & $4.55_{2.23}^{2.80}-1.47_{1.39}^{3.62}-17.21_{570}^{1.96}$ & $78.00_{0.27}^{0.33}$ \\
\hline 16 & $0.23_{0.17}^{0.27}-0.00_{0.00}^{0.00}-7.21_{0.74}^{0.60}$ & $2.03_{0.57}^{0.67}-19.48_{1.80}^{1.30}-0.85_{0.83}^{4.55}$ & $0.00_{0.00}^{0.00}-0.00_{0.00}^{0.00}-0.00_{0.00}^{0.21}$ & $75.33_{0.36}^{0.27}$ \\
\hline 17 & $1.64_{0.48}^{0.40}-0.00_{0.00}^{0.00}-1.51_{1.25}^{1.94}$ & $1.71_{0.63}^{0.69}-23.99_{1.97}^{2.80}-7.55_{7.60}^{14.59}$ & $0.58_{0.47}^{0.69}-0.00_{0.00}^{0.00}-18.86_{4.09}^{5.28}$ & $82.47_{1.05}^{1.08}$ \\
\hline 18 & $1.61_{051}^{0.40}-0.00_{0.00}^{0.00}-0.00_{0.00}^{0.00}$ & $2.87_{1.05}^{0.71}-19.12_{1.03}^{1.78}-0.41_{0.41}^{1.90}$ & $0.00_{0.00}^{0.00}-0.03_{0.03}^{1.12}-28.26_{128}^{1.48}$ & $74.24_{0.24}^{0.21}$ \\
\hline 19 & $0.00_{0.00}^{0.00}-0.00_{0.00}^{0.00}-10.42_{0.77}^{0.75}$ & $2.72_{0.53}^{0.05}-17.76_{1.15}^{1.03}-0.00_{0.00}^{0.00}$ & $0.00_{0.00}^{0.00}-0.00_{0.00}^{0.00}-6.16_{1.46}^{1.24}$ & $83.65_{0.21}^{0.14}$ \\
\hline 20 & $1.96_{035}^{0.39}-0.00_{0.00}^{0.00}-0.00_{0.00}^{0.00}$ & $0.00_{0.00}^{0.00}-2.84_{0.43}^{0.48}-3.80_{0.69}^{0.80}$ & $0.00_{0.00}^{0.00}-0.00_{0.00}^{0.00}-11.58_{0.62}^{0.00}$ & $86.71_{1.02}^{0.91}$ \\
\hline 21 & $0.14_{0.13}^{0.33}-0.00_{0.00}^{0.00}-1.11_{1.11}^{4.09}$ & $1.37_{0.57}^{0.65}-16.62_{3.51}^{0.13}-5.51_{4.54}^{0.69}$ & $0.00_{0.00}^{0.00}-0.33_{0.33}^{3.89}-8.29_{2.43}^{0.62}$ & $68.29_{0.021}^{0.02}$ \\
\hline 22 & $0.00_{0.00}^{0.00}-0.00_{0.00}^{0.00}-11.71_{1.60}^{1.60}$ & $0.00_{0.00}^{0.000}-11.63_{213}^{1.78}-3.04_{254}^{4.20}$ & $0.00_{0.00}^{0.00}-0.00_{0.00}^{0.00}-0.00^{0.000}$ & $79.96_{029}^{0.26}$ \\
\hline 23 & $2.75_{036}^{0.30}-0.00_{0.00}^{0.00}-0.18_{0.71}^{0.76}$ & $0.74_{054}^{0.83}-9.09_{123}^{1.07}-6.89_{1}^{1.01}$ & $0.00_{0.00}^{0.00}-0.00_{0.00}^{0.00}-14.88^{1.03}$ & $69.41_{0}^{0:}$ \\
\hline 24 & $0.28_{0 \rightarrow 2}^{0.32}-0.00_{0.00}^{0.00}-13.61_{0.88}^{0.71}$ & $0.13^{0.40}-20.26_{25}^{1.84}-4.88^{2.82}$ & $0.00_{0.00}^{0.00}-0.00_{0.00}^{0.00}-0.99^{1.680}$ & $82.50_{0.36}^{0.34}$ \\
\hline 25 & $0.00_{0.00}^{0.00}-0.12_{0.12}^{0.70}-1.16_{081}^{0.968}$ & $3.42^{1.72}-9.49^{1.70}-1.35^{1.90} 0^{1.94}$ & $0.00_{0.00}^{0.11}-0.00_{0.00}^{0.00}-16.32^{1.69}$ & $79.60_{147}^{0.95}$ \\
\hline 26 & $1.82^{0.36}-0.00^{0.00}-1.28^{0.69}$ & $2.53^{0.49}-26.80_{2}^{2.18}-6.03^{3.50}$ & $0.00_{0.00}^{0.00}-0.00_{0.00}^{0.00}-11.68^{1.91}$ & $79.95^{0.49}$ \\
\hline 27 & $0.07^{0.29}-1.95^{1.12}-10.13^{1.85}$ & $0.12^{0.40}-16.58_{1.11}^{0.89}-0.72^{2.45}$ & $0.04^{0.34}-0.00^{0.00}-8.93_{182}^{1.78}$ & $82.74_{055}^{0.42}$ \\
\hline 28 & $3.44_{037}^{0.34}-0.00_{0.00}^{0.07}-4.75_{222}^{3.00}$ & $0.37_{031}^{0.11}-20.22_{161}^{1.21}-4.86_{465}^{7.795}$ & $0.00_{0.00}^{0.04}-0.00_{0.00}^{0.00}-5.04_{2.32}^{0.02}$ & $60.03^{2.52}$ \\
\hline 29 & $0.00_{0.00}^{0.00}-0.00^{0.00}-0.84^{2.53}$ & $2.22_{138}^{1.53}-14.13_{8.09}^{6.81}-0.03^{0.68}$ & $0.00^{0.00}-0.00^{0.00}-2.96^{2.00}$ & $83.09^{2.52}$ \\
\hline 30 & $0.00_{0.00}^{0.00}-1.20_{087}^{0.00}-7.92^{1.81}$ & $0.06_{0.06}^{6.38}-19.99_{167}^{1.60}-4.85_{349}^{2.83}$ & $0.00_{0.00}^{8.00}-0.00_{0.00}^{8.00}-7.14_{129}^{1.35}$ & $76.51_{126}^{0.99}$ \\
\hline 31 & $0.00_{0.00}^{0.00}-0.00_{0.00}^{0.00}-3.51_{154}^{2.51}$ & $2.54_{133}^{1.34}-11.85_{3.04}^{2.80}-0.01_{0.00}^{0.00}$ & $0.00^{0.00}-0.00^{0.00}-0.08^{2.05}$ & $90.67^{0.20}$ \\
\hline 32 & $0.06^{0.026}-0.00^{0.00}-0.87^{1.07}$ & $1.06_{060}^{0.33}-18.16_{180}^{1.04}-0.00_{0.00}^{0.01}$ & $0.03^{0.00}-0.00^{0.00}-2.00-2.64^{0.06}=08$ & $83.69^{0.322}$ \\
\hline 33 & $1.33^{0.32}-0.00^{0.00}-6.53^{0.73}$ & $2.04^{0.51}-13.43_{187}^{2.34}-31.80_{2.67}^{2.60}$ & $0.13^{0.52}-0.00_{0.00}^{0.00}-4.01_{2}^{1.76}$ & $80.38_{026}^{0.32}$ \\
\hline 34 & $3.80_{0.03}^{1.00}-0.00^{8.00}-0.00^{8.000}$ & $3.10_{108}^{1.33}-14.11_{457}^{4.57}-5.03^{7.94}$ & $0.14^{1.50}-4.75^{5.80}-21.50^{7.0 .07}$ & $47.88_{476}^{3.51}$ \\
\hline 35 & $1.62_{1.06}^{1.93}-0.08_{0.08}^{1.90}-0.00_{0.00}^{0.00}$ & $0.39^{1.43}-23.24_{575}^{5.50}-5.04_{4.50}^{11.83}$ & $0.06_{0.06}^{0.135}-0.27_{0.27}^{5.52}-24.35_{719}^{6.072}$ & $83.15_{170}^{0.762}$ \\
\hline 36 & $0.00_{0.00}^{0.00}-0.06_{0.06}^{2.85}-3.91_{1.85}^{2.00}$ & $0.00_{0.00}^{0.00}-0.30_{0.30}^{5.155}-29.73_{5.13}^{5.50}$ & $2.11_{132}^{2.03}-0.00_{0.00}^{0.00}-0.00_{0.00}^{0.00}$ & $80.36_{6.69}^{8.39}$ \\
\hline 37 & $2.17_{1.41}^{1.26}-2.01_{1.88}^{3.80}-3.07_{3.01}^{5.46}$ & $0.51_{0.47}^{0.99}-21.49_{10.46}^{7.85}-0.69_{0.69}^{7.39}$ & $0.03_{0.03}^{0.90}-0.11_{0.11}^{3.15}-9.17_{6.43}^{7.1 .17}$ & $82.01_{4.34}^{2.89}$ \\
\hline 38 & $0.22_{0.21}^{0.56}-1.07_{0.88}^{1.89}-0.23_{0.23}^{1.65}$ & $3.07_{1.09}^{1.12}-9.80_{3.99}^{3.15^{\circ}}-2.31_{2.20}^{7.26^{9}}$ & $0.00_{0.00}^{0.00}-0.00_{0.00}^{0.00}-0.45_{0.45}^{0.43}$ & $79.88_{0.84}^{0.66}$ \\
\hline 39 & $0.30_{0.30}^{1.28}-0.13_{0.13}^{2.10}-2.76_{2.23}^{3.60}$ & $0.03_{0.03}^{1.16}-11.81_{5,56}^{7.76}-4.13_{4.03}^{14.24}$ & $0.57_{0.56}^{3.17}-0.00_{0.00}^{0.00}-0.29_{0.29}^{6.29}$ & $81.79_{2}^{2}$ \\
\hline 40 & $1.45_{0.90}^{1.33}-0.00_{0.00}^{0.00}-17.67_{4.02}^{4.03}$ & $0.08_{0.08}^{0.69}-20.62_{3.98}^{4.81}-0.97_{0.97}^{7.41}$ & $0.30_{0.30}^{1.90}-0.00_{0.00}^{0.00}-23.33_{6.42}^{5.47}$ & $86.15_{0.97}^{1.09}$ \\
\hline 41 & $9.57_{5.60}^{2.45}-0.66_{0.65}^{3.31}-6.53_{4.85}^{9.81}$ & $0.05_{0,05}^{1.26}-6.46_{3,95}^{4.198}-12.14_{7.13}^{6.96}$ & $0.04_{0.04}^{0.74}-0.17_{0.17}^{2.06}-3.73_{3.22}^{6.452}$ & $68.59_{1.53}^{2.84}$ \\
\hline 42 & $0.01_{0.01}^{0.54}-2.26_{146}^{1.95}-1.53_{1.44}^{3.39}$ & $0.79_{0.59}^{1.11}-6.97_{2.80}^{2.91}-0.83_{0.82}^{7.21}$ & $0.09_{0.09}^{2.010}-11.51_{2.95}^{3.72}-2.07_{2.02}^{5.63}$ & $70.33_{3.01}^{1.81}$ \\
\hline 43 & $0.01_{0.01}^{0.34}-3.26_{102}^{0.49}-0.14_{0.14}^{1.84}$ & $0.44_{0.40}^{0.98}-10.04_{433}^{2.84}-3.14_{299}^{6.48}$ & $1.80_{1.16}^{1.38}-3.18_{2.47}^{2 . .91}-0.00_{0.00}^{0.00}$ & $81.10_{0.48}^{0.51}$ \\
\hline 44 & $0.01_{0.01}^{0.41}-0.03_{0.03}^{0.94}-0.00_{0.00}^{0.00}$ & $0.17_{0.99}^{0.99}-9.47_{420}^{4.08}-0.02_{0.02}^{0.069}$ & $4.42_{1.80}^{1.05}-7.92_{5.23}^{5.59}-1.87_{185}^{7.30}$ & $69.58_{4.47}^{2.68}$ \\
\hline 45 & $0.00_{0.00}^{8.00}-0.00_{0.00}^{8.00}-0.00_{0.00}^{8.00}$ & $1.23_{076}^{0.73}-0.18_{0.18}^{2.12}-0.04_{0.04}^{2.64}$ & $0.00_{0.00}^{0.00}-0.00_{0.00}^{0.00}-0.00_{0.00}^{0.00}$ & 55.2 \\
\hline 46 & $0.21_{0.19}^{0.62}-7.93_{2.96}^{1.99}-3.39_{2.66}^{4.03}$ & $0.03_{003}^{0.86}-19.91_{3.33}^{3.42}-8.11_{542}^{6.64}$ & $0.00_{000}^{0.00}-0.01_{0.01}^{0.00}-4.71_{345}^{4.50}$ & $60.58_{0.63}^{0.618}$ \\
\hline 47 & $0.00_{0.00}^{0.00}-0.10_{0.00}^{1.00}-7.17^{1.63}$ & $0.18_{017}^{0.53}-8.60_{245}^{1.89}-4.30_{3.83}^{9.842}$ & $2.89_{0.98}^{0.88}-1.24_{114}^{2.95}-2.73_{240}^{3.55}$ & 52.995 \\
\hline 48 & $0.33^{0.80}-0.02^{0.606}-4.82^{4.54}$ & $0.84^{1.95}-0.42^{4.47}-48.19^{9.63}$ & $1.47^{2.15}-0.34_{035}^{6.62}-0.79^{6.39}$ & $89.87_{3}^{1}$ \\
\hline 49 & $0.65^{1.39}-0.01^{8.00}-0.00^{0.00}$ & $0.19^{4.110}-3.72_{369}^{30.02}-29.42_{110}^{25.88}$ & $1.96_{184}^{4.45}-0.00^{8.00}-0.18^{4.20}$ & $77.89^{6.70}$ \\
\hline 50 & $2.25_{1.26}^{1.26}-0.95^{3.89}-0.57_{0.07}^{4.00}$ & $7.20_{210}^{2.22}-10.92_{736}^{9.89}-0.01^{0.001}$ & $0.20_{020}^{2.384}-0.59^{6.44}-29.27^{8.780}$ & $81.33^{2.61}$ \\
\hline 51 & $7.61_{0.64}^{1.53}-0.00^{0.000}-0.03_{0.03}^{0.57}$ & $0.00^{0.00}-6.76_{070}^{0.83}-0.05^{4.10}$ & $0.02^{0.78}-0.00_{0.00}^{0.00}-6.87^{2.54}$ & $63.78^{0.40}$ \\
\hline 52 & $0.36_{0.34}^{0.847}-2.13_{177}^{4.21}-10.83_{582}^{4.537}$ & $0.94_{0.90}^{1.89}-3.09^{3.28}-6.74_{496}^{5.415}$ & $3.05_{1.83}^{1.92}-0.07^{2.22}-3.27_{2}^{2.67}$ & $65.99^{2.12}$ \\
\hline 53 & $3.98_{0.48}^{0.44}-0.00_{0.00}^{0.00}-0.00_{0.00}^{0.00}$ & $0.96_{0.39}^{0.48}-17.04_{135}^{4.57}-0.00_{0.00}^{0.00}$ & $2.31_{0.91}^{1.1 .14}-0.00_{0.00}^{0.00}-27.17_{2.83}^{2.81}$ & $21.98_{1.93}^{4.74}$ \\
\hline 54 & $7.91_{3.02}^{1.04}-0.51_{0.51}^{2.27}-0.49_{0.49}^{4.111}$ & $0.16_{0.16}^{2.02}-0.95_{0.94}^{5.09}-29.41_{22.43}^{8.83}$ & $0.02_{0.02}^{2.01}-0.00_{0.00}^{0.00}-23.13_{13.30}^{9.06}$ & $80.83_{1275}^{2.74}$ \\
\hline 55 & $0.01_{0.01}^{0.22}-1.84_{1.45}^{1.51}-2.72_{2.23}^{3.09}$ & $0.01_{0.01}^{0.29}-0.27_{0.27}^{1.84}-18.74_{4.10}^{3.93}$ & $0.21_{0.12}^{1.04}-4.18_{2.42}^{2.92}-1.30_{1.30}^{4.363}$ & $72.36_{0.58}^{0.75}$ \\
\hline 56 & $2.07_{0.99}^{1.115}-2.33_{2.13}^{3.08}-5.83_{4.30}^{4.38}$ & $0.08_{0.08}^{0.79}-5.14_{1.60}^{2.05}-0.08_{0.08}^{7.26}$ & $0.12_{0.12}^{1.20}-0.01_{0.01}^{0.42}-5.53_{277}^{3.29}$ & \\
\hline 57 & $4.32_{1.60}^{1.03}-0.00_{0.00}^{0.00}-0.00_{0.00}^{0.00}$ & $2.76_{1.56}^{1.440}-14.26_{6.04}^{6.32}-7.32_{6.06}^{8.52}$ & $6.36_{2.14}^{2.08}-0.00_{0.00}^{0.001}-30.32_{10.15}^{8.87}$ & $53.30_{10.45}^{4.72}$ \\
\hline
\end{tabular}

Notes. The last column gives the continuum flux contribution, listed as a percentage of the total integrated flux over the full wavelength range. 